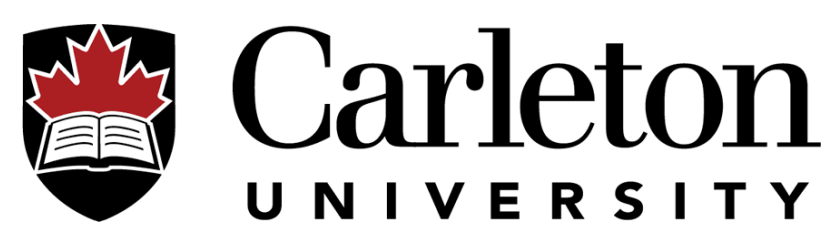

\title{
Susceptibility Modelling of Space-Time Modulated Metasurfaces
}

\author{
VILLE TIUKUVAARA \\ DEPARTMENT OF ELECTRONICS \\ Carleton University
}
thesis submitted to the Faculty of Graduate and Postdoctoral Affairs in partial fulfillment of the requirements for the degree of
Master of Applied SCIENCE, M. A.Sc.
(ELECTRICAL AND COMPUTER ENGINEERING)
August 2021

(C) Ville Tiukuvaara, 2021. 


\section{Carleton University}

This thesis titled:

Susceptibility Modelling of Space-Time Modulated Metasurfaces

presented by: TIUKUVAARA Ville

to obtain the degree of : Masters of Applied Science, M.A.Sc.

Evaluated by the thesis committee consisting of:
Dr. GUNUPUDI Pavan, Ph. D., Chair
Dr. GUPTA Shulabh, Ph. D., Research Supervisor
Dr. SMY Tom J., Ph. D., Research Supervisor
Dr. MCNAMARA Derek, Ph. D., External Member
Dr. MCGARRY Steve, Ph. D., Internal Member 


\begin{abstract}
Electromagnetic metasurfaces (MSs) present a challenge for modelling and numerical simulations, as they are inherently "multi-scale". Thus, equivalent models-namely zero-thickness sheets described by surface susceptibilities-are usually employed for numerical simulations. At the same time, space-time varying media have received interest in the past years due to the exciting phenomena they facilitate (e.g. non-reciprocity). This thesis primarily considers the intersection of these two research directions: the modelling of MSs with periodic variation in space and/or time, allowing a Floquet expansion to be used to calculate scattered fields in an efficient way. This thesis also presents susceptibility extractions for some canonical structures, including a ground plane with a dielectric cover layer. Finally, experimental results are shown for MSs which were fabricated, operating in the Ka-band, as a demonstration of the developed Floquet method. These were measured using a near-field (NF) system developed at Carleton University, as a contribution of this thesis.
\end{abstract}




\section{TABLE OF CONTENTS}

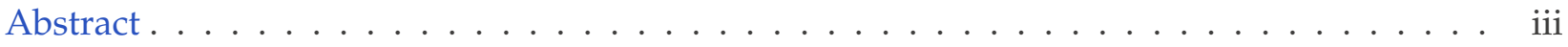

TABLE OF CONTENTS $\ldots \ldots \ldots \ldots \ldots \ldots \ldots \ldots \ldots \ldots$ iv

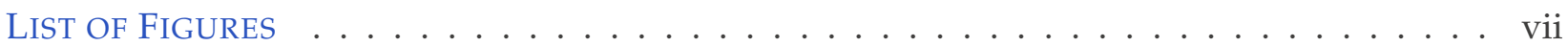

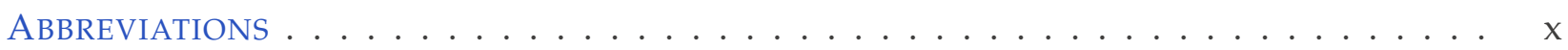

Chapter 1 Thesis Motivation, Objectives \& Contributions . . . . . . . . . 1

1.1 Thesis Objectives $\ldots \ldots \ldots \ldots \ldots \ldots \ldots \ldots$

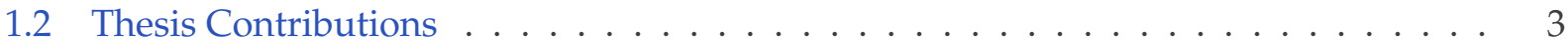

Chapter 2 Surface Susceptibility Modelling of Metasurfaces . . . . . . . . . 5

2.1 Introduction $\ldots \ldots \ldots \ldots \ldots \ldots \ldots \ldots \ldots \ldots \ldots \ldots \ldots \ldots \ldots \ldots \ldots \ldots$

2.2 Zero-Thickness MS Models . . . . . . . . . . . . . . . . . . . 6

2.2.1 Impedance Boundary Conditions (IBCs) . . . . . . . . . . . . . 6

2.2.2 Generalized Sheet Transition Conditions (GSTCs) . . . . . . . . . . . . . . 7

2.3 Plane Wave Illumination for a Uniform MS . . . . . . . . . . . . . . . . 8

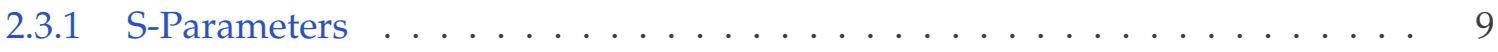

2.3.2 Susceptibility Extraction Procedure . . . . . . . . . . . . . . . 10

2.4 Example: A Curvilinear Metasurface Composed of Metal Loops . . . . . . . . . . . 11

2.4 .1 Unit Cell and Extraction . . . . . . . . . . . . . . . . . . 12

2.4 .2 Parametric Study . . . . . . . . . . . . . . . . . . . . 13

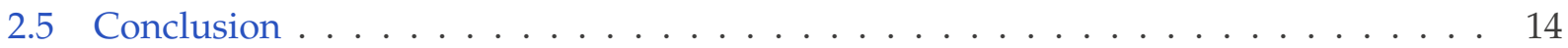


Chapter 3 Surface Susceptibility Extractions of Canonical Structures ～. 15

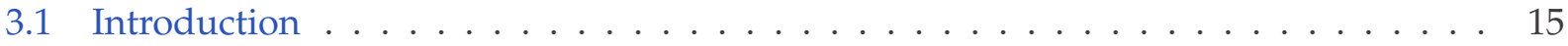

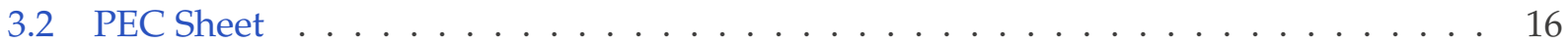

3.3 Dielectric Sheet . . . . . . . . . . . . . . . . . . . . . 17

3.4 PEC Sheet with Cover Layer . . . . . . . . . . . . . . . . . . . . . 18

3.5 Sub-wavelength Resonator on a Dielectric Slab . . . . . . . . . . . . . . . . 21

3.6 Numerical Demontration: Parabolic Reflector . . . . . . . . . . . . . . . . . . . . . 22

3.7 Conclusions \& Future Work . . . . . . . . . . . . . . . . . . . . . . . 24

Chapter 4 Floquet Analysis of Space-Time Modulated Metasurfaces . . . . . 25

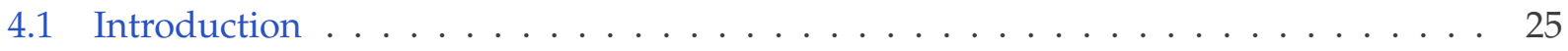

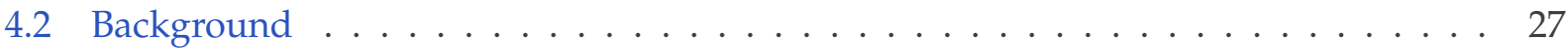

4.2 .1 Linear Time Invariant $(\mathrm{LTI}) \mathrm{MSs} \ldots \ldots \ldots \ldots$. . . . . . . . . . . . 27

4.2.2 Linear Time-Variant (LTV) MSs . . . . . . . . . . . . . . . . . . . . . 29

4.2 .3 Lorentzian Susceptibilities . . . . . . . . . . . . . . . . . . . . . . 30

4.3 Harmonic Expansion of Equations and Fields . . . . . . . . . . . . . . . . 31

4.4 Matrix Formulation . . . . . . . . . . . . . . . . . . . . 33

4.4 .1 Lorentzian Formulation . . . . . . . . . . . . . . . . . . . . 33

4.4 .2 "Dispersionless" Formulation . . . . . . . . . . . . . . 35

4.4 .3 Extension to an Arbitrary Incident Field . . . . . . . . . . . . . . . 35

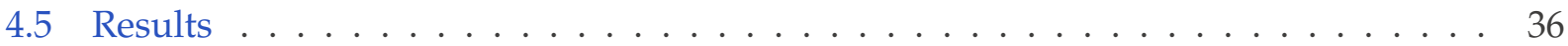

4.5 .1 Space-Only Modulation . . . . . . . . . . . . . . . . . . 36

4.5 .2 Time-Only Modulation . . . . . . . . . . . . . . . . . . 39

4.5 .3 Space-Time Modulation . . . . . . . . . . . . . . . . 42

4.6 Conclusions \& Future Work . . . . . . . . . . . . . . . . . . . . . . . . 44

Chapter 5 Experimental Near-Field Characterization of Metasurfaces _ . . 46

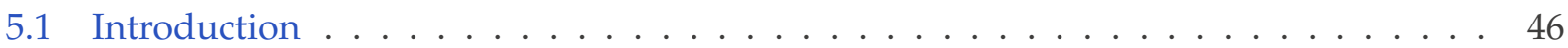

5.2 Development of a Custom NF System . . . . . . . . . . . . . . . . . . 48

5.2 .1 System Overview . . . . . . . . . . . . . . . . . . . . . . . 48

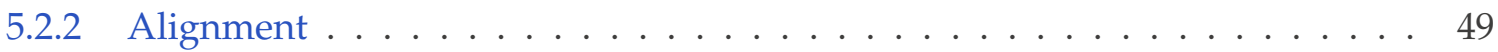

5.2 .3 Graphical User Interface $(\mathrm{GUI}) \ldots \ldots \ldots$. . . . . . . . . . . . 50 
5.3 Fabrication and Measurement of a Periodic Ka-Band MS . . . . . . . . . . . . . 50

5.3 .1 Unit Cell Design . . . . . . . . . . . . . . . . . . . . 50

5.3 .2 Periodic MS Design . . . . . . . . . . . . . . . . . . . . . . . 51

5.3 .3 Measurement ........................... 53

5.4 Incident Field Reconstruction using a Single Horn Antenna . . . . . . . . . . . . . . 56

5.4 .1 Practical Metasurface Illumination . . . . . . . . . . . . . . . . . . 57

5.4 .2 Construction of an Arbitrary Incident Field . . . . . . . . . . . . . . . . 59

5.4 .3 Example: A Parabolic Surface . . . . . . . . . . . . . . . . . . . . . . 60

5.5 Conclusions \& Future Work . . . . . . . . . . . . . . . . . . . . . . . 62

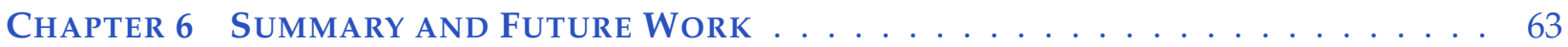

6.1 Key Conclusions . . . . . . . . . . . . . . . . . . . . . . . 63

6.2 Future Work . . . . . . . . . . . . . . . . . . . . . . . . 64

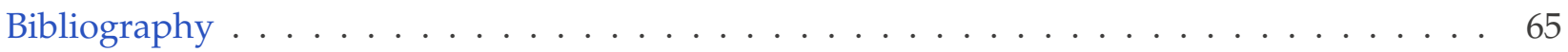




\section{LIST OF FIGURES}

Example of a space-modulated Ka-band MS. . . . . . . . . . . . . . . 6

A depiction of the electric field orientations for TE and TM fields, with forwards and backwards plane wave illumination of a planar structure (e.g. a metasurface). Propagation is in the $x-z$ plane, with the metasurface in the $x-y$ plane.

A simple unit cell used here as an example, with a strong normal polarization. a) Unit cell model, having a period $p=3 \mathrm{~mm}$ and a substrate with $\epsilon_{r}=3.4$ and $\tan \delta=0.002$. The metal is copper. b) Extracted susceptibilities for TE polarization, using $(2.8) \ldots \ldots \ldots \ldots$. . . . . . . . . . . . 12

2.4 A comparison of the S-parameters predicted with the extracted susceptibilities in Figure $2.3 \mathrm{~b}$ to the simulated values from Ansys HFSS. . . . . . . . . . . 13

2.5 Comparison between the magnitude of the total E-fields calculated using BEM-GSTC and FEM-HFSS for a semi-circular metasurface as the curvature radius $r_{0}$ was decreased from $\infty$ (i.e. a flat surface), where $N$ is the number of cells. There is a plane wave incident at $\theta=30^{\circ}$ in the $x y$-plane, and the structure is uniform in the $z$ direction. . . . . . . . . . . . . . . . . . . . . . 14

3.1 The reflection and transmission through a lossy dielectric sheet was calculated analytically to compare with the mapped susceptibilities, with and without the normal components. . . . . . . . . . . . .

3.2 The reflection from a PEC sheet with a lossy dielectric cover layer was calculated analytically to compare with the mapped susceptibilities, with and without the normal components. . . . . . . . . . . . . . . . 20

3.3 A deeply-subwavelength reflective unit cell was designed using an electric dipole, loaded with a lumped inductor $(L)$ on top of a Rogers RO4003C substrate $\left(\epsilon_{r}=3.55, \tan \delta_{d}=0.0027\right)$ that is on a PEC ground-plane. . . . . . . . 

a focal length of $10 \mathrm{~cm}$ with forwards illumination. . . . . . . . . . . . 23

An illustration of scattering from from a space-time modulated MS with a spatial period $L$, where a TE plane wave incident at an angle $\theta_{0}$ produces reflected and transmitted fields. Surface susceptibilities model the structure as having zero thickness $d$, while the surface extends infinitely along $x$ and $y$. Space-time modulation produces scattered harmonics $(m, n)$ that exist at discrete frequencies $\omega(n)$ (hence the $k(n)$ circles) and discrete transverse spatial frequencies $k_{x}(m)$ (horizontal lines). At each $k_{x}(m)$, there are an infinite number of time harmonics with different $k(n)$ and thus different angles of scattering $\theta(m, n)$

A plane wave with $\omega_{0}=2 \pi(230 \mathrm{THz})$ is normally incident on a MS where the Lorentzian resonant frequencies are spatially modulated as $\zeta_{\{\mathrm{ee}, \mathrm{mm}\}}^{\{y y, x x\}}(x)=$

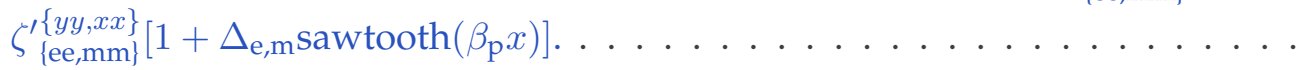

A Gaussian beam with waist $5 \lambda$ and amplitude $E_{\mathrm{g}}$ is incident at $\theta_{0}=10^{\circ}$ onto a modulated surface with $\zeta_{\{\mathrm{ee}, \mathrm{mm}\}}^{\{y y, x x\}}(x)=\zeta_{\{\mathrm{ee}, \mathrm{mm}\}}^{\{y y, x\}}\left[1+\Delta_{\mathrm{e}, \mathrm{m}} \cos \left(\beta_{\mathrm{p}} x\right)\right]$ with $\beta_{\mathrm{p}}=k_{0} / 5.76$ and $\Delta_{\mathrm{e}, \mathrm{m}}=0.2 \ldots \ldots \ldots \ldots$

A surface composed of capacitively-loaded loops on a polyimide (PI) film $\left(\epsilon_{r}=3.4\right.$, loss tangent 0.002) was modelled in HFSS, where in each cell the side length of a MIM capacitor $l$ was modulated. A comparison is made to the Floquet method. . . . . . . . . . . . . . . . . . . . . .

4.6 Shows the reflected field at the surface $\left(z=0^{-}\right)$for a uniform surface that is time-modulated with an incident plane wave at $\omega_{0}=2 \pi(130 \mathrm{THz})$ that is far-away from the resonant frequencies of the surface susceptibilities. . . . . Shows three cases of uniform MSs that are time-modulated with $\zeta_{\{\mathrm{ee}, \mathrm{mm}\}}^{\{y y, x\}}(t)=$ $\zeta_{\{\mathrm{ee}, \mathrm{mm}\}}^{\{y y, x x\}}\left[1+\Delta_{\mathrm{e}, \mathrm{m}} \cos \left(\omega_{\mathrm{p}} t\right)\right]$. In each of the three cases $(\mathrm{a}-\mathrm{c})$, the incident field frequency and modulation parameters are varied. . . . . . . . . .

4.8 To examine the discrepancy between the FDTD and Floquet results in Fig. 4.7c, the consistency of the solutions was considered. The FDTD discrepancy is larger than the discrepancy of the Floquet result, while the latter improves as the number of harmonics $(2 N+1)$ is increased. . . . . . . . . . . . . 42

4.9 Reciprocal space-time modulation where space and time dependencies are decoupled. . . . . . . . . . . . . . . . .

4.10 Non-reciprocal space-time modulated MS wherez space and time dependencies are coupled. . . . . . . . . . . . . . . . . . 44 
5.3 The CNC frame allows for scanning along three axes, controlled by stepper motors, while end-stop sensors allow for precise calibration of the position. . 49 Alignment of the antennas and MS, primarily using a 3-plane laser. . . . . . . 49 Shows the three tabs in the custom Python GUI used to run automated NF scans. . . . . . . . . . . . . . . . . . . . . 50

A two-layer metasurface was designed to have a magnetic resonance in the Ka-band. (a) Unit cell with identical metal dog-bones on either side of a Rogers RO3010 substrate $\left(\epsilon_{r}=11.2, \tan \delta=0.0022\right)$. (b) Depiction of formation of $x$-directed magnetic dipole $\left(M_{x}\right)$, with antisymmetric $y$-directed surface currents on opposite dipoles $\left( \pm J_{\mathrm{s}, y}\right)$. (c) Extracted susceptibilities for three different values of $l$. (d) Susceptibilities for extractions at all $l$ values, at the design frequency. . . . . . . . . . . . . . . . . . 5

A MS was fabricated using a periodic "square wave" modulation of $l$. (a) Fabricated MS. (b) Profile of $l$ over a single period. (c) Profile of susceptilibilities over a single period. . . . . . . . . . . . . . . . . . 52

5.8 A MS was fabricated using a periodic "triangle wave" modulation of $l$. (a) Fabricated MS. (b) Profile of $l$ over a single period. (c) Profile of susceptilibilities over a single period. . . . . . . . . . . . . . . . . 52 Incident field. . . . . . . . . . . . . . . . . . . . . . 53

5.10 Transmitted fields from the MS shown in Figure 5.7 with square wave modulation, using the incident field from Figure 5.9 . . . . . . . . . . . . . . 55

5.11 Transmitted fields from the MS shown in Figure 5.8 with triangle wave modulation, using the incident field from Figure 5.9. . . . . . . . . . . 56

5.12 The Eravant SAR-2013-28-S2 horn antenna was simulated using HFSS, and is well-approximated using a Gaussian beam having the parameters $w_{0}=$ $1.00 \mathrm{~mm}, x_{\mathrm{bw}}=0 \mathrm{~cm}$, and $z_{\mathrm{bw}}=-4.14 \mathrm{~cm} \ldots \ldots \ldots \ldots$

5.13 An incident field $\mathbf{E}_{\mathrm{i}}\left(\left\{p_{n}\right\}, x, z\right)$ generated with the superposition of $N=8$ illuminations, with the objective of uniform amplitude and phase (i.e. nor-

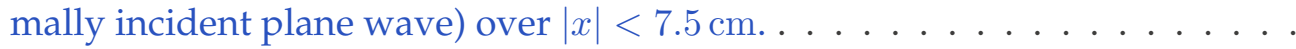

5.14 A focusing MS was designed and then illuminated with three different incident fields in $(\mathrm{b}-\mathrm{d}) . \ldots \ldots$. . . . . . . . . . . . . . . . . . . 61 


\section{ABBREVIATIONS}

1-D 1-Dimensional

2-D 2-Dimensional

3-D 3-Dimensional

BEM Boundary Element Method

CNC Computer Numerical Control

CSV Comma-Separated Values

DC Direct Current

DMC Digital Motion Controller

EM Electromagnetic

FEM Finite Element Method

FDFD Finite-Difference Frequency-Domain

FDTD Finite-Difference Frequency-Domain

GSTC Generalized Sheet Transition Conditions

GUI Graphical User Interface

HK Holloway-Kuester

HFSS High Frequency Structure Simulator

IBC Impedance Boundary Conditions

IE Integral Equation

LTI Linear Time-Invariant

LNA Low Noise Amplifier

LTV Linear Time-Variant

MIM Metal-Insulator-Metal

MARS Metamaterials and Antennas Research Squad

MS Metasurface

NF Near Field

PCB Printed Circuit Board

PEC Perfect Electric Conductor

PI Polyimide 
PMC Perfect Magnetic Conductor

RF Radio Frequency

Rx Receive

TE Transverse Electric

TM Transverse Magnetic

TS Tretyakov-Simovski

Tx Transmit

USB Universal Serial Bus

VNA Vector Network Analyzer 


\section{conew 1}

\section{Thesis Motivation, Objectives \& CONTRIBUTIONS}

The emergence of metasurfaces (MSs) over the past two decades has spawned numerous papers devoted to theoretical developments, experimental demonstrations, and enterprising visions in ways that they could be applied in practical ways. The possibility of using metasurfaces to provide field transformations has been extensively studied. For example, a metasurface can be designed to provide arbitrary control over amplitude, phase, and polarization of the scattered fields [1] —and even for multiple channels, i.e. different incident fields [2]. Static MSs have been applied to different regimes of the electromagnetic spectrum: from flat lenses for optics [3], to the gain-improvement of microwave antennas [4]. Static metasurfaces can also implement electrical tunability, which may play a role in controlling the propagation environment in future wireless communications [5].

While static metasurfaces have afforded this useful ability to manipulate the scattered fields, new research directions continue to provide rich new physical effects. One recent research direction involves introducing time variation to the MS, which breaks the limit of reciprocity without the need for bulky magnetic materials. For example, the MS properties might be manipulated using active components such as PIN diodes or varactors, which are controlled by external circuitry [6]. This enables applications including isolators, circulators, and frequency mixers, and opens up possibilities like using space-time diffraction patterns as channels for wireless communications [7] and non-reciprocal beam steering for full-duplex communications [8]. A MS which has both spatial and temporal variation can be called a space-time modulated MS.

The primary focus of this thesis is the development of an efficient numerical method for analyzing periodic space-time modulated MSs, using a zero-thickness surface susceptibility model and the generalized sheet transistion conditions (GSTCs). The GSTCs model a MS as a sheet of electric and magnetic polarization densities, and provide a much faster iterative design flow compared 
to full-wave simulations of the MS at a microscopic level. ${ }^{1}$ The GSTCs have been implemented in a number of numerical methods (e.g. finite-difference time-domain, the finite element method, and integral equation methods), but the consideration of a MS which is periodic in both space and time allows for a semi-analytical method based on a Floquet expansion, which is developed and verified in this thesis.

At the same time, there has historically been some confusion regarding the application and validity of surface susceptibilities. For example, some authors have opted to treat metasurfaces as thin volumetric slabs having some equivalent electrical properties. This is in fact not physically appropriate, which has been discussed in foundational papers like [9, 10], which promote zerothickness surface susceptibilities as a more appropriate and rigorous approach. What has not been sufficiently addressed is whether susceptibilities can be used to model fully-reflective MSs. A simple example is a ground plane with a dielectric coating. Is it possible to model the behaviour of both electrically-isolated sides of this structure using a single set of susceptibilities? This question is fundamentally important.

Finally, no metasurface design is complete without experimental verification. In particular, the near-fields (NF) of a MS can provide great insight into the functionality of a metasurface. For example, the functionality of a MS lens can be verified by measuring the NF phase profile. NF measurements are typically performed with custom automated systems-such a system was needed for the Metamaterials and Antennas Research Group (MARS) in Carleton University and was developed as a part of this thesis.

\subsection{Thesis Objectives}

This thesis has three main objectives:

1. Develop surface susceptibility models for fully-reflective MSs, which are true constitutive parameters independent of the incident field. This requires consideration of the full tensorial surface susceptibilities, including bi-anisotropy. Building up to a MS consisting of an array of electric dipoles on top of a ground-plane with a dielectric cover layer, the use of susceptibilities as compact models for full-wave simulations is also clarified.

2. Develop a fast and semi-analytical method for calculating the scattered field from a spacetime modulated MS, described by periodic surface susceptibilities. For rigor, the frequency dispersion of the MS is modelled using susceptibilities having Lorentzian frequency profiles. The method is verified with comparison to alternative simulations. Furthermore, the method is extended to allow for non-periodic incident fields.

3. Develop a Ka-band NF measurement system, for the characterization of transmissive MSs. This includes writing Python code which automates the movement of a probe antenna, and

${ }^{1}$ The meaning of microscopic is clarified in Chapter 2. 
captures S-parameters from a vector network analyzer (VNA). It has an easy-to-use graphical user interface (GUI) to make it accessible to other users.

\subsection{Thesis Contributions}

This thesis has consequently led to the following contributions and publications:

1. Examination of surface susceptibilities as compact models for fully reflective and curvilinear surfaces.

[1] V. Tiukuvaara, T. J. Smy, K. Achouri, and S. Gupta, "Surface Susceptibilities as Compact Full-Wave Simulation Models of Fully-Reflective Volumetric Metasurfaces," IEEE Trans Antennas Propag, under review.

[2] V. Tiukuvaara, T. J. Smy, and S. Gupta, “Investigation into Curvilinear Metasurfaces using IE-GSTCs with Normal Surface Polarizabilities," accepted for presentation in 2021 IEEE AP-S Symp Antennas Propag.

2. Development of a scattered field calculation method based on Floquet harmonic expansion for space-time modulated MSs.

[3] V. Tiukuvaara, T. J. Smy, and S. Gupta, "Floquet Analysis of Space-Time Modulated Metasurfaces with Lorentz Dispersion," IEEE Trans Antennas Propag, 2021, doi: 10.1109/ TAP.2021.3070718.

[4] V. Tiukuvaara, T. J. Smy, and S. Gupta, "Metasurface Modeling of Periodic Diffraction Gratings based on Generalized Sheet Transition Conditions (GSTCs)," in 2020 14th Eur Conf Antennas Propag (EuCAP).

3. Development of a boundary element method (BEM) simulator incorporating complete tensorial dipolar surface susceptibilities.

[5] T. J. Smy, V. Tiukuvaara, and S. Gupta, "IE-GSTC Metasurface Field Solver using Surface Susceptibility Tensors with Normal Polarizabilities," IEEE Trans Antennas Propag, under review.

4. Experimental near-field measurement techniques for MSs.

[6] V. Tiukuvaara, K. Wang, T. J. Smy, and S. Gupta, "Metasurface Near-field Measurements with Incident Field Reconstruction using a Single Horn Antenna," IEEE Instrum Meas Mag, under review.

In addition to these contributions with regards to the outlined objectives, during the thesis research I also contributed to the following publications, which consider the mapping between eigenmode and driven simulations: 
[7] J. G. N. Rahmeier, V. Tiukuvaara, and S. Gupta, “Complex Eigenmodes and Eigenfrequencies in Electromagnetics," IEEE Trans Antennas Propag, vol. 69, no. 8, 2021.

[8] J. G. N. Rahmeier, V. Tiukuvaara, and S. Gupta, "Mapping Between Complex Eigenmodes and Complex Propagation Constant for Uniform Rectangular Metallic Waveguides," in 2020 14th Eur Conf Antennas Propag (EuCAP). 
Chapter 2

\section{SURface SusceptibiLity MODELLiNG OF METASURFACES}

\subsection{Introduction}

MSs are generally constructed from arrays of sub-wavelength scattering particles forming a sheet having finite thickness, akin to periodic arrays of atoms forming crystals in solid state physics [11]. In this work, I consider MSs where the particles (i.e. unit cells) are on a rectangular lattice. Figure 2.1 shows an example of a Ka-band MS composed of cells with two copper electric dipoles on either side of a substrate. ${ }^{1}$ The MS can be non-uniform; the example shows a periodic modulation where the dipole length $l$ is varied in the $x$-direction, with a profile $l(x)$.

Upon interacting with an incident electromagnetic field, electric (and magnetic) polarizations are induced in the array; these have rapid variations on the scale of the unit cell, with corresponding electromagnetic near-fields. These are the microscopic fields. To be clear, these microscopic fields are what are observed in and around each of the dielectric or metallic particles within each unit cell. It is convenient to use alternative homogenized models for MSs, which involve an averaging of the polarization densities over the area of a unit cell $[9,10]$. This homogenization "smoothes out" any variations smaller than a unit cell, and produces macroscopic fields. The macropscopic fields can be used to produce boundary conditions-such as the GSTCs-which predict the macroscopic reflected and transmitted fields. As an analogy, this homogenization is akin to the use of relative permittivities to describe dielectric materials, where in reality polarized dielectrics have strong microscopic electric field variations on the scale of the constitutive molecules or atoms. The primary difference is the scale of averaging.

To this end, there are a few main approaches that have been used as boundary conditions for metasurfaces [12]:

\footnotetext{
${ }^{1}$ The analysis and measurement of this MS is in Chapter 5.
} 


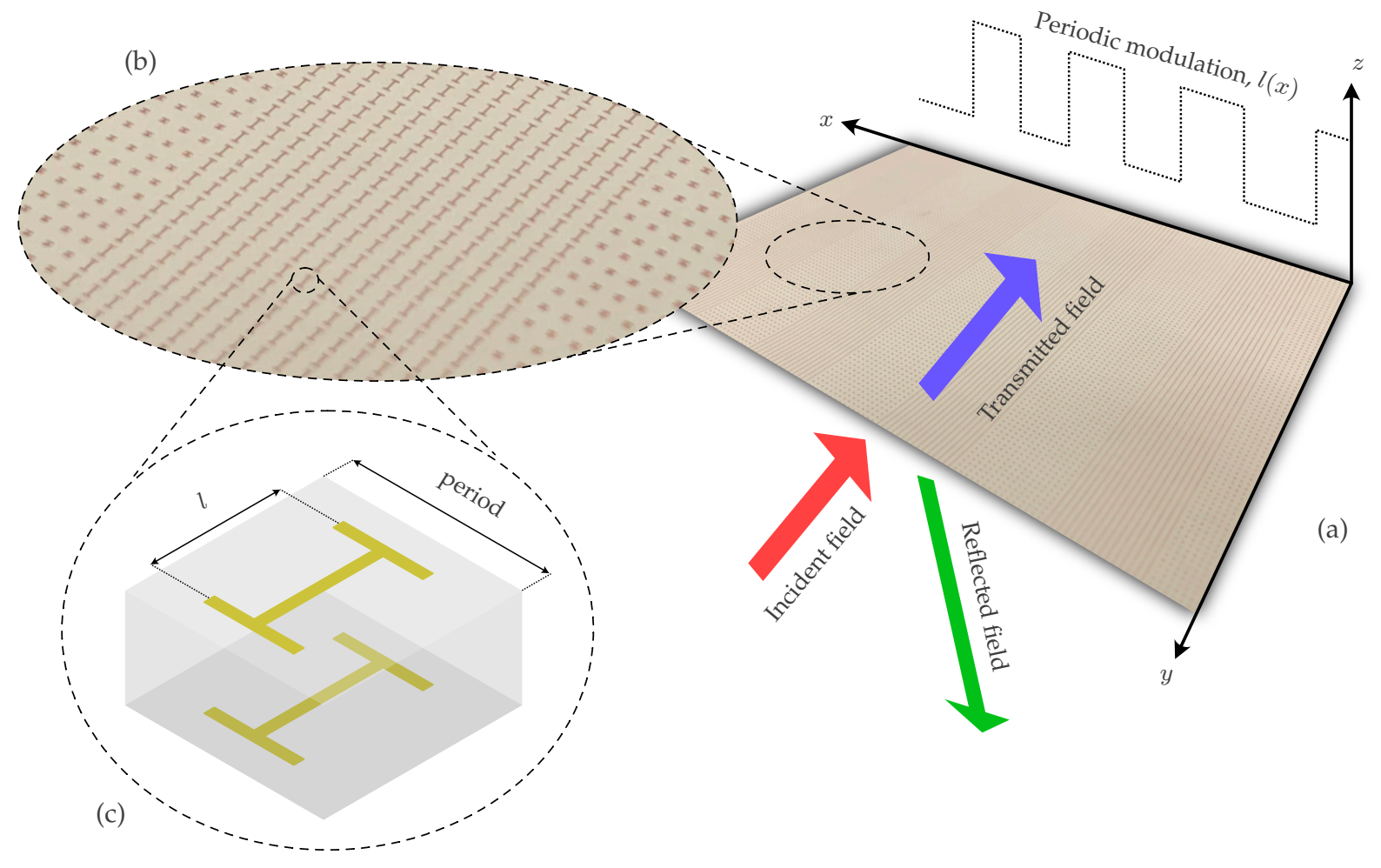

Figure 2.1 Example of a space-modulated Ka-band MS. (a) Depiction of incident, reflected, and transmitted fields from MS with 1-D modulation in $x$-direction, $l(x)$. (b) Expanded view of fabricated MS, showing the top layer with a nonuniform array of dipoles. (c) Schematic view of a single deeply-sub-wavelength unit cell, indicating the parameter $l$ which is modulated.

1. Impedance Boundary Conditions (IBCs)

2. Generalized Sheet Transition Conditions (GSTCs) with surface susceptibilities

These are well known, and each has advantages and disadvantages. In the next section, I briefly outline these approaches, while the remainder of this thesis considers the GSTCs.

\subsection{Zero-Thickness MS Models}

\subsubsection{Impedance Boundary Conditions (IBCs)}

The IBCs follow from the conventional boundary conditions, whereby the discontinuity of the tangential fields is related to surface currents [12]:

$$
\begin{aligned}
\hat{\mathbf{n}} \times \Delta \mathbf{H} & =\mathbf{J}_{s} \\
\Delta \mathbf{E} \times \hat{\mathbf{n}} & =\mathbf{K}_{s}
\end{aligned}
$$


where $\mathbf{E}$ and $\mathbf{H}$ are the macroscopic fields while $\mathbf{J}_{s}$ and $\mathbf{K}_{s}$ are electric and magnetic surface currents, respectively, and $\Delta \phi=\phi_{\mathrm{t}}-\left(\phi_{\mathrm{i}}+\phi_{\mathrm{r}}\right)(\phi \in\{\mathbf{E}, \mathbf{H}\})$ are the difference in fields across the boundary. ${ }^{2}$ I define $\hat{\mathbf{n}}= \pm \hat{\mathbf{z}}$ as being the surface normal in the direction of incidence, directed from the side on which the incident field is present to the transmission side as in Figure 2.2 (to be elucidated shortly). Meanwhile, the currents are induced by the tangential components of fields averaged across the surface [12]:

$$
\begin{aligned}
\mathbf{J}_{s} & =\overline{\bar{Z}}_{\mathrm{e}}^{-1} \cdot \mathbf{E}_{\mathrm{av} \|} \\
\mathbf{K}_{s} & =\overline{\bar{Z}}_{\mathrm{m}} \cdot \mathbf{H}_{\mathrm{av} \|}
\end{aligned}
$$

with $\phi_{\mathrm{av}}=\frac{1}{2}\left(\phi_{\mathrm{t}}+\phi_{\mathrm{i}}+\phi_{\mathrm{r}}\right), \overline{\bar{Z}}_{\mathrm{e}}\left(\overline{\bar{Z}}_{\mathrm{m}}\right)$ being an electric (magnetic) sheet impedance, and where $\|$ denotes the projection to the surface ( $x-y$ plane).

The IBCs have a distinct advantage in that impedances can be simply cascaded to model multilayered metasurfaces, akin to series and shunt elements on a transmission line [2]. However, a major drawback is that these impedances are not true constitutive parameters, since they are in general dependent on the incident field. For example, if a MS is illuminated with an incident plane wave, the impedances will in general depend on the angle of incidence.

\subsubsection{Generalized Sheet Transition Conditions (GSTCs)}

On the other hand, surface susceptibilities are true constitutive parameters. I will use the formulation presented in [13]. The boundary conditions for the components of the fields tangential to the metasurface - the GSTCs-are

$$
\begin{gathered}
\hat{\mathbf{n}} \times \Delta \mathbf{H}=j \omega \mathbf{P}_{\|}-\hat{\mathbf{n}} \times \nabla M_{z} \\
\Delta \mathbf{E} \times \hat{\mathbf{n}}=j \omega \mu_{0} \mathbf{M}_{\|}+\epsilon_{0}^{-1} \hat{\mathbf{n}} \times \nabla P_{z}
\end{gathered}
$$

where $\mathbf{P}=\mathbf{P}_{\|}+\hat{\mathbf{z}} P_{z}$ and $\mathbf{M}=\mathbf{M}_{\|}+\hat{\mathbf{z}} M_{z}$ are the electric and magnetic surface polarization densities, respectively. The MS is assumed to be surrounded by free space (permittivity $\epsilon_{0}$, permeability $\mu_{0}$, impedance $\eta_{0}$, and wavenumber $k_{0}$ ).

Meanwhile, the polarization densities are related to the averaged electric fields by the constitutive relations (HK model)

$$
\begin{aligned}
& \mathbf{P}=\epsilon_{0} \overline{\bar{\chi}}_{\mathrm{ee}} \cdot \mathbf{E}_{\mathrm{av}}+\epsilon_{0} \eta_{0} \overline{\bar{\chi}}_{\mathrm{em}} \cdot \mathbf{H}_{\mathrm{av}} \\
& \mathbf{M}=\overline{\bar{\chi}}_{\mathrm{mm}} \cdot \mathbf{H}_{\mathrm{av}}+\eta_{0}^{-1} \overline{\bar{\chi}}_{\mathrm{me}} \cdot \mathbf{E}_{\mathrm{av}}
\end{aligned}
$$

There are four sets of tensors, $\overline{\bar{\chi}}$, for a total of 36 constitutive parameters. These can model the normal part of the polarization densities, along with bianisotropy (the em/me tensors).

\footnotetext{
${ }^{2}$ In keeping with other literature in the context of MSs, the total field on the transmission side is just $\mathbf{E}_{\mathrm{t}}$. For example, in the absence of a MS, $\mathbf{E}_{\mathrm{t}}$ is the incident field on the transmission side.
} 


\subsection{Plane Wave Illumination for a Uniform MS}

Many of the tensor components in (2.4) can be eliminated or simplified due to reciprocity, symmetry, or energy conservation, depending on the particular surface. This work will consider surfaces which involve no polarization conversion, and which are reciprocal. This simplifies the tensors to $[14,15]$

$$
\begin{aligned}
\overline{\bar{\chi}}_{\mathrm{ee}}=\left(\begin{array}{ccc}
\chi_{\mathrm{ee}}^{x x} & 0 & 0 \\
0 & \chi_{\mathrm{ee}}^{y y} & 0 \\
0 & 0 & \chi_{\mathrm{ee}}^{z z}
\end{array}\right) & \overline{\bar{\chi}}_{\mathrm{mm}}=\left(\begin{array}{ccc}
\chi_{\mathrm{mm}}^{x x} & 0 & 0 \\
0 & \chi_{\mathrm{mm}}^{y y} & 0 \\
0 & 0 & \chi_{\mathrm{mm}}^{z z}
\end{array}\right) \\
\overline{\bar{\chi}}_{\mathrm{em}}=\left(\begin{array}{ccc}
0 & \chi_{\mathrm{em}}^{x y} & 0 \\
\chi_{\mathrm{em}}^{y x} & 0 & 0 \\
0 & 0 & 0
\end{array}\right) & \overline{\bar{\chi}}_{\mathrm{me}}=-\overline{\bar{\chi}}_{\mathrm{em}}^{T}
\end{aligned}
$$

With the 8 unique terms retained in these selected tensors, no assumptions have been made regarding energy conservation, and there is a possibility for omega-type bianisotropy [16].

Since the susceptibilities in (2.5) do not convert polarization, TE and TM illuminations can be considered separately, as depicted in Figure 2.2. With the periodicity of the surface being subwavelength, no higher-order diffraction orders are generated [17] and under oblique plane wave illumination at $\theta$, there will be reflected and transmitted plane waves at the same angle, following standard Snell's laws and depicted in Figure 2.2. Henceforth, the analysis is restricted to plane waves propagating in the $x-z$ plane for simplicity, unless otherwise noted.

The left and right sides of the (infinite) surface can be denoted as "ports", labelled 1 and 2, respectively, such that e.g. $S_{21}^{\{\mathrm{TE}, \mathrm{TM}\}}$ denotes transmission in the forwards direction and $S_{12}^{\{\mathrm{TE}, \mathrm{TM}\}}$ in the backwards direction. With this convention, the forms of the fields for an excitation of port 1 (i.e.

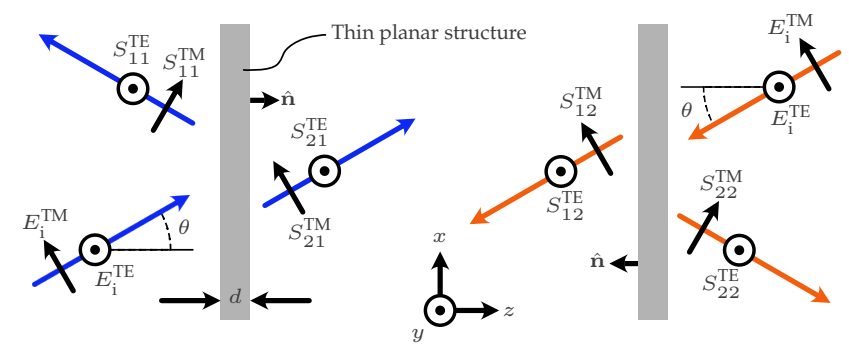

(a) Forwards illumination $(+z) \quad$ (a) Backwards illumination $(-z)$

Figure 2.2 A depiction of the electric field orientations for TE and TM fields, with forwards and backwards plane wave illumination of a planar structure (e.g. a metasurface). Propagation is in the $x-z$ plane, with the metasurface in the $x-y$ plane. 
incoming wave from the left side or forwards illumination), with $\hat{\mathbf{n}}=+\hat{\mathbf{z}}$, is

$$
\begin{gathered}
\mathbf{E}_{\mathrm{i}}=\left[\begin{array}{lll}
E_{1}^{\mathrm{TM}} \cos \theta & E_{1}^{\mathrm{TE}} & -E_{1}^{\mathrm{TM}} \sin \theta
\end{array}\right]^{T} e^{-j k_{0}(x \sin \theta+z \cos \theta)} \\
\mathbf{E}_{\mathrm{r}}=\left[\begin{array}{lll}
S_{11}^{\mathrm{TM}} E_{1}^{\mathrm{TM}} \cos \theta & S_{11}^{\mathrm{TE}} E_{1}^{\mathrm{TE}} & S_{11}^{\mathrm{TM}} E_{1}^{\mathrm{TM}} \sin \theta
\end{array}\right]^{T} e^{-j k_{0}(x \sin \theta-z \cos \theta)} \\
\mathbf{E}_{\mathrm{t}}=\left[\begin{array}{lll}
S_{21}^{\mathrm{TM}} E_{1}^{\mathrm{TM}} \cos \theta & S_{21}^{\mathrm{TE}} E_{1}^{\mathrm{TE}} & -S_{21}^{\mathrm{TM}} E_{1}^{\mathrm{TM}} \sin \theta
\end{array}\right]^{T} e^{-j k_{0}(x \sin \theta+z \cos \theta)}
\end{gathered}
$$

where the vectors have been written as columns with $T$ denoting a transpose. $E_{1}^{\mathrm{TE}}$ is the amplitude of the TE part of the incident plane wave, while $E_{1}^{\mathrm{TM}}$ is the amplitude of the TM part. Meanwhile, an excitation of port 2 (i.e. incoming wave from the right side or backwards illumination), with $\hat{\mathbf{n}}=-\hat{\mathbf{z}}$, is

$$
\begin{gathered}
\mathbf{E}_{\mathrm{i}}=\left[\begin{array}{lll}
E_{2}^{\mathrm{TM}} \cos \theta & E_{2}^{\mathrm{TE}} & -E_{2}^{\mathrm{TM}} \sin \theta
\end{array}\right]^{T} e^{-j k_{0}(x \sin \theta-z \cos \theta)} \\
\mathbf{E}_{\mathrm{r}}=\left[\begin{array}{lll}
S_{22}^{\mathrm{TM}} E_{2}^{\mathrm{TM}} \cos \theta & S_{22}^{\mathrm{TE}} E_{2}^{\mathrm{TE}} & S_{22}^{\mathrm{TM}} E_{2}^{\mathrm{TM}} \sin \theta
\end{array}\right]^{T} e^{-j k_{0}(x \sin \theta+z \cos \theta)} \\
\mathbf{E}_{\mathrm{t}}=\left[\begin{array}{lll}
S_{12}^{\mathrm{TM}} E_{2}^{\mathrm{TM}} \cos \theta & S_{12}^{\mathrm{TE}} E_{2}^{\mathrm{TE}} & -S_{12}^{\mathrm{TM}} E_{2}^{\mathrm{TM}} \sin \theta
\end{array}\right]^{T} e^{-j k_{0}(x \sin \theta-z \cos \theta)}
\end{gathered}
$$

Meanwhile, the magnetic fields are simply found from Faraday's equation:

$$
\mathbf{H}_{\{\mathrm{i}, \mathrm{r}, \mathrm{t}\}}=\frac{1}{j \omega \mu} \nabla \times \mathbf{E}_{\{\mathrm{i}, \mathrm{r}, \mathrm{t}\}}
$$

\subsubsection{S-Parameters}

Substituting (2.6) along with (2.5) into (2.3) and (2.4), it is possible to solve for the S-parameters. These are

$$
\begin{aligned}
S_{\{11,22\}}^{\mathrm{TE}}(\theta) & =\frac{2 j k}{\xi_{\mathrm{TE}}}\left(\zeta_{\mathrm{TE}} \mp 2 \chi_{\mathrm{em}}^{y x} \cos \theta-\chi_{\mathrm{mm}}^{x x} \cos ^{2} \theta\right) \\
S_{\{21,12\}}^{\mathrm{TE}}(\theta) & =\frac{\cos \theta}{\xi_{\mathrm{TE}}}\left(4+k^{2}\left[\left(\chi_{\mathrm{em}}^{y x}\right)^{2}+\chi_{\mathrm{mm}}^{x x} \zeta_{\mathrm{TE}}\right]\right) \\
S_{\{11,22\}}^{\mathrm{TM}}(\theta) & =\frac{2 j k}{\xi_{\mathrm{TM}}}\left(\zeta_{\mathrm{TM}} \mp 2 \chi_{\mathrm{em}}^{x y} \cos \theta-\chi_{\mathrm{ee}}^{x x} \cos ^{2} \theta\right) \\
S_{\{21,12\}}^{\mathrm{TM}}(\theta) & =\frac{\cos \theta}{\xi_{\mathrm{TM}}}\left(4+k^{2}\left[\left(\chi_{\mathrm{em}}^{x y}\right)^{2}+\chi_{\mathrm{ee}}^{x x} \zeta_{\mathrm{TM}}\right]\right)
\end{aligned}
$$


where the top and bottom signs ( $\mp$ ) are taken for $11 / 21$ and 22/12, respectively, and

$$
\begin{gathered}
\xi_{\{\mathrm{TE}, \mathrm{TM}\}}=-4 \cos \theta-2 j k\left(\zeta_{\{\mathrm{TE}, \mathrm{TM}\}}+\chi_{\{\mathrm{mm}, \mathrm{ee}\}}^{x x} \cos ^{2} \theta\right) \\
+k^{2} \cos \theta\left(\zeta_{\{\mathrm{TE}, \mathrm{TM}\}} \chi_{\{\mathrm{mm}, \mathrm{ee}\}}^{x x}+\left(\chi_{\mathrm{em}}^{\{y x, x y\}}\right)^{2}\right) \\
\zeta_{\{\mathrm{TE}, \mathrm{TM}\}}=\chi_{\{\mathrm{ee}, \mathrm{mm}\}}^{y y}+\chi_{\{\mathrm{mm}, \mathrm{ee}\}}^{z z} \sin ^{2} \theta
\end{gathered}
$$

Note in particular that the bianisotropic terms $\chi_{\mathrm{em}}^{\{y x, x y\}}$ [and implicitly terms in $\overline{\bar{\chi}}_{\mathrm{me}}$ following (2.5b)] lead to an asymmetry in reflection when the direction of illumination is flipped, as noted in [14]. If $\chi_{\mathrm{em}}^{y x}=0$ or $\chi_{\mathrm{em}}^{x y}=0$, then $S_{\{11\}}^{\mathrm{TE}}=S_{\{22\}}^{\mathrm{TE}}$ or $S_{\{11\}}^{\mathrm{TM}}=S_{\{22\}}^{\mathrm{TM}}$, respectively. At the same time, $S_{\{21\}}^{\mathrm{TE}}=S_{\{12\}}^{\mathrm{TE}}$ and $S_{\{21\}}^{\mathrm{TM}}=S_{\{12\}}^{\mathrm{TM}}$ always hold true, which is a result of enforcing reciprocity in (2.5).

\subsubsection{Susceptibility Extraction Procedure}

While (2.7) allows calculating the scattered fields given susceptibilities, it is also possible to extract the susceptibilities for a given MS. As given in (2.5), there are eight unique unknown susceptibilities, which requires eight equations for finding a solution. Since forwards and backwards illumination cases in (2.7b) [and (2.7d)] the the same, it is not sufficient to know the S-parameters at a single angle, $\theta$. However, it is possible to use two angles of incidence to provide eight independent equations to solve for the susceptibilities.

Firstly, let $\theta=0^{\circ}$. This eliminates the normal components in (2.7f) and it is possible to solve for the tangential components:

$$
\begin{gathered}
\chi_{\mathrm{ee}}^{x x}=\frac{2 j\left(\left[S_{21}^{\mathrm{TM}}(0)\right]^{2}-S_{11}^{\mathrm{TM}}(0)\left[S_{22}^{\mathrm{TM}}(0)-1\right]+S_{22}^{\mathrm{TM}}(0)-1\right)}{k_{0}\left(1+2 S_{21}^{\mathrm{TM}}(0)+\left[S_{21}^{\mathrm{TM}}(0)\right]^{2}-S_{11}^{\mathrm{TM}}(0) S_{22}^{\mathrm{TM}}(0)\right)} \\
\chi_{\mathrm{ee}}^{y y}=\frac{2 j\left(\left[S_{21}^{\mathrm{TE}}(0)\right]^{2}-S_{11}^{\mathrm{TE}}(0)\left[S_{22}^{\mathrm{TE}}(0)-1\right]+S_{22}^{\mathrm{TE}}(0)-1\right)}{k_{0}\left(1+2 S_{21}^{\mathrm{TE}}(0)+\left[S_{21}^{\mathrm{TE}}(0)\right]^{2}-S_{11}^{\mathrm{TE}}(0) S_{22}^{\mathrm{TE}}(0)\right)} \\
\chi_{\mathrm{mm}}^{x x}=-\frac{2 j\left(S_{11}^{\mathrm{TE}}(0)\left[S_{22}^{\mathrm{TE}}(0)+1\right]-\left[S_{21}^{\mathrm{TE}}(0)\right]^{2}+S_{22}^{\mathrm{TE}}(0)+1\right)}{k_{0}\left(-S_{11}^{\mathrm{TE}}(0) S_{22}^{\mathrm{TE}}(0)+\left[S_{21}^{\mathrm{TE}}(0)\right]^{2}+2 S_{21}^{\mathrm{TE}}(0)+1\right)} \\
\chi_{\mathrm{mm}}^{y y}=-\frac{2 j\left(S_{11}^{\mathrm{TM}}(0)\left[S_{22}^{\mathrm{TM}}(0)+1\right]-\left[S_{21}^{\mathrm{TM}}(0)\right]^{2}+S_{22}^{\mathrm{TM}}(0)+1\right)}{k_{0}\left(-S_{11}^{\mathrm{TM}}(0) S_{22}^{\mathrm{TM}}(0)+\left[S_{21}^{\mathrm{TM}}(0)\right]^{2}+2 S_{21}^{\mathrm{TM}}(0)+1\right)}
\end{gathered}
$$




$$
\begin{aligned}
& \chi_{\mathrm{em}}^{y x}=-\frac{2 j\left(S_{11}^{\mathrm{TE}}(0)-S_{22}^{\mathrm{TE}}(0)\right)}{k_{0}\left(-S_{11}^{\mathrm{TE}}(0) S_{22}^{\mathrm{TE}}(0)+\left[S_{21}^{\mathrm{TE}}(0)\right]^{2}+2 S_{21}^{\mathrm{TE}}(0)+1\right)} \\
& \chi_{\mathrm{em}}^{x y}=\frac{2 j\left(S_{11}^{\mathrm{TM}}(0)-S_{22}^{\mathrm{TM}}(0)\right)}{k_{0}\left(-S_{11}^{\mathrm{TM}}(0) S_{22}^{\mathrm{TM}}(0)+\left[S_{21}^{\mathrm{TM}}(0)\right]^{2}+2 S_{21}^{\mathrm{TM}}(0)+1\right)}
\end{aligned}
$$

Next, using a separate experiment at $\theta \neq 0^{\circ}$ to get $S_{11}^{\{\mathrm{TE}, \mathrm{TM}\}}(\theta)$, and already having the tangential susceptibilities from (2.8a-f), the normal components can be found:

$$
\chi_{\mathrm{ee}}^{z z}=\frac{j \csc ^{2}(\theta)\left(\begin{array}{c}
2 k_{0}\left(-\chi_{\mathrm{mm}}^{y y}+2 \cos (\theta) \chi_{\mathrm{em}}^{x y}+\cos ^{2}(\theta) \chi_{\mathrm{ee}}^{x x}\right) \\
+S_{11}^{\mathrm{TM}}(\theta)\left[j k_{0}^{2} \cos (\theta)\left(\chi_{\mathrm{mm}}^{y y} \chi_{\mathrm{ee}}^{x x}+\left(\chi_{\mathrm{em}}^{x y}\right)^{2}\right)\right. \\
\left.+2 k_{0}\left(\chi_{\mathrm{mm}}^{y y}+\cos ^{2}(\theta) \chi_{\mathrm{ee}}^{x x}\right)-4 j \cos (\theta)\right]
\end{array}\right)}{j \csc ^{2}(\theta)\left(\begin{array}{c}
2 k_{0}\left(\chi_{\mathrm{ee}}^{y y}-2 \cos (\theta) \chi_{\mathrm{em}}^{y x}-\cos ^{2}(\theta) \chi_{\mathrm{mm}}^{x x}\right) \\
+S_{11}^{\mathrm{TE}}(\theta)\left[j k_{0}^{2} \cos (\theta)\left(\chi_{0} \cos (\theta) \chi_{\mathrm{ee}}^{x x}-2 j\right]+2 j\right) \\
\left.+2 k_{0}\left(\chi_{\mathrm{ee}}^{y x}+\cos ^{2}(\theta) \chi_{\mathrm{mm}}^{x x}\right)-4 j \cos (\theta)\right]
\end{array}\right)}
$$

\subsection{Example: A Curvilinear Metasurface Composed of Metal Loops}

To demonstrate the susceptibility extraction process, I will show a numerical example using a metallic loop with Metal-Insulator-Metal (MIM) gap capacitors to tune the resonance, as shown in Figure 2.3a. Subsequently, I will show the accuracy of the susceptibilities in two ways. Firstly, the susceptibilities will be shown to predict the S-parameters, regardless the angle of incidence, confirming that they are appropriate constitutive parameters. Secondly, I will utilize the Integral Equation (IE)-based field solver proposed in [18] to compute the scattered fields from a finite-sized curvilinear metasurface built from the simple physical unit cell.

This second point merits some elaboration. GSTCs have generally been used with planar (flat) MSs, which are easy to analyze in Cartesian coordinates. Recently, some works have considered conformal (curvilinear) MSs, which are promising for applications including wearable devices and cloaking $[19,20]$. However, there are two key questions which we believe have not been sufficiently addressed. Firstly, are GSTCs valid along a curvilinear boundary? [19] seems to suggest that the conventional GSTCs cannot be straightforwardly applied in this case. Secondly, are the susceptibilities extracted from an infinite and a flat array of unit cells [12] accurate when applied to a curvilinear surface? Hence, the aforementioned second verification with a curvilinear MS has 
great practical value.

\subsubsection{Unit Cell and Extraction}

As noted, the unit cell consists of a metallic loop with MIM capacitors, which is excited with TE fields in the $x y$-plane. The cell is deeply-subwavelength with a period $p=\lambda_{0} / 10$ (at $f_{0}=10 \mathrm{GHz}$ ) and was designed to resonate in the X-band, by adjusting the dimensions of the loop and the capacitors. For simplicity, only TE polarization and the associated susceptibilities are considered here (TM polarization does not provide additional insight).

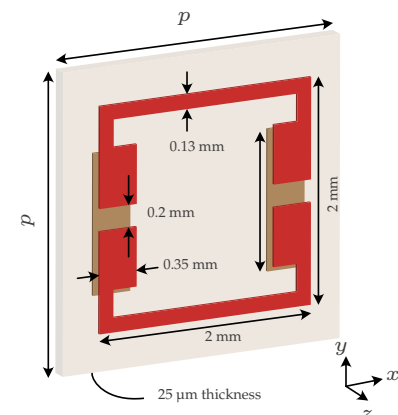

(a)

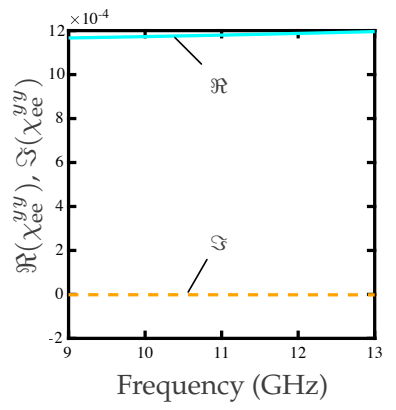

(b)

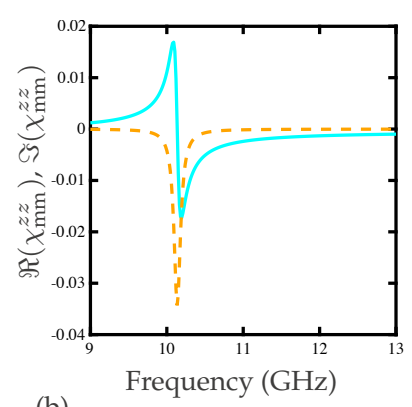

)

Figure 2.3 A simple unit cell used here as an example, with a strong normal polarization. a) Unit cell model, having a period $p=3 \mathrm{~mm}$ and a substrate with $\epsilon_{r}=3.4$ and $\tan \delta=0.002$. The metal is copper. b) Extracted susceptibilities for TE polarization, using (2.8).

The unit cell was simulated in Ansys HFSS with periodic boundaries, such that it was equivalent to an infinite-sized uniform MS. Using two angles of incidence $\left(\theta \in\left\{0^{\circ}, 60^{\circ}\right\}\right)$, the susceptibilities relevant for TE $\left(\chi_{\mathrm{ee}}^{y y}, \chi_{\mathrm{mm}}^{x x}, \chi_{\mathrm{mm}}^{z z}\right.$, and $\left.\chi_{\mathrm{em}}^{y x}\right)$ could be found from (2.8) and are shown in Figure 2.3. The extraction procedure revealed a strong normal magnetic polarization $\chi_{\mathrm{mm}}^{z z}$ along with tangential electric response $\chi_{\mathrm{ee}}^{y y}$, while $\chi_{\mathrm{mm}}^{x x} \approx 0$ and $\chi_{\mathrm{em}}^{y x} \approx 0$. The normal magnetic polarization is expected, since the loop supports rotating electric currents in the $x-z$ plane, which corresponds to a $z$-directed magnetic dipole. The negligible bi-anisotropic term results from the forwards illumination and backwards illumination producing the same scattering [i.e. $S_{11}^{\mathrm{TE}}(0)-S_{22}^{\mathrm{TE}}(0)=0$ in (2.8e)].

Next, the S-parameters can be predicted using (2.7). In this case, they reduce to

$$
\begin{aligned}
& S_{11,22}^{\mathrm{TE}}(\theta)=\frac{-j \frac{k_{0}}{2 \cos \theta}\left(\chi_{\mathrm{ee}}^{y y}+\chi_{\mathrm{mm}}^{z z} \sin ^{2} \theta\right)}{1+j \frac{k_{0}}{2 \cos \theta}\left(\chi_{\mathrm{ee}}^{y y}+\chi_{\mathrm{mm}}^{z z} \sin ^{2} \theta\right)} \\
& S_{21,12}^{\mathrm{TE}}(\theta)=\frac{1}{1+j \frac{k_{0}}{2 \cos \theta}\left(\chi_{\mathrm{ee}}^{y y}+\chi_{\mathrm{mm}}^{z z} \sin ^{2} \theta\right)}
\end{aligned}
$$

Fig. 2.4 shows very good agreement between (2.9) and the simulated S-parameters, which were used to extract the susceptibilities. This should obviously hold true for the angles at which the extraction was performed $\left(\theta \in\left\{0^{\circ}, 60^{\circ}\right\}\right)$, but more importantly the susceptibilities are able to 
model the MS regardless of the angle of incidence, making them constitutive parameters. In fact, the extracted surface susceptibilities can very accurately model the angular response of the surface to a near-grazing angle of incidence.
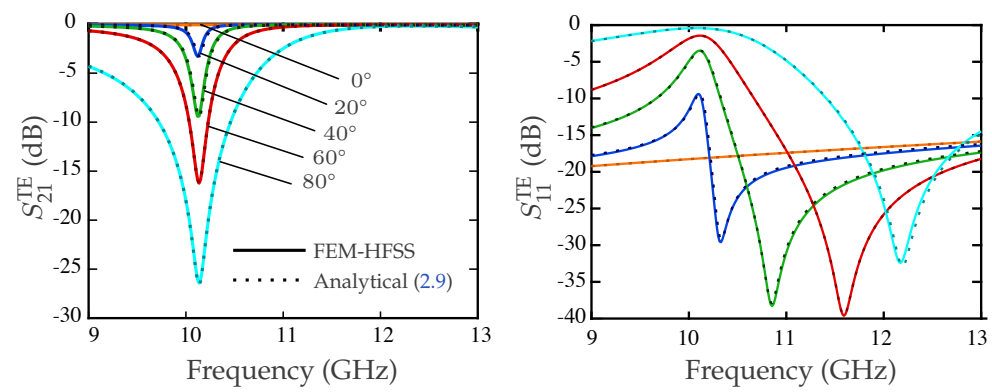

Figure 2.4 A comparison of the S-parameters predicted with the extracted susceptibilities in Figure 2.3b to the simulated values from Ansys HFSS.

\subsubsection{Parametric Study}

Having found the surface susceptibilities, $\chi_{\mathrm{mm}}^{z z}$ and $\chi_{\mathrm{ee}}^{y y}$, I now consider whether this representation can be used with the GSTCs to model a curvilinear MS. To systematically consider the curvature effect, I selected a semi-circular metasurface, which has a constant curvature, quantified by its radius, $r_{0}$, where $r_{0}=\infty$ corresponds to a flat metasurface. I constructed this semi-circular shaped metasurface using the unit cell of Fig. 2.3a for various values of $r_{0}$, thereby requiring different number of unit cells $N$ due to its finite size. It was then simulated in Ansys FEM-HFSS to obtain the total fields for a specified incident field. Next, an equivalent zero-thickness sheet model was constructed using the extracted surface susceptibilities from Fig. 2.3b, and analyzed using the BEM-GSTC solver of [18] using the same incident fields. The BEM code implements the GSTCs and the complete tensorial surface susceptibilities. By decreasing $r_{0}$, we can now observe how the curvature affects the agreement between BEM-GSTCs and the complete structure using FEMHFSS.

For the study, I start with a flat surface consisting of $N=12$, shown in Fig. 2.5a. To have a significant induced normal magnetic polarization, a plane wave is incident at $\theta=30^{\circ}$. An excellent agreement is seen between the fields generated by the two solvers, except for the microscopic fields around each resonator which as expected, cannot be captured by the homogenized susceptibilities. In particular, the BEM-GSTCs are able to successfully capture the diffraction around the ends of the finite flat surface. This agreement can further be seen more clearly in the top of Fig. 2.5e, where the total fields are compared along an observation circle around the metasurface. Next, the radius is gradually decreased for three cases $r_{0}=\left\{\lambda_{0} / 1.5, \lambda_{0} / 2, \lambda_{0} / 3\right\}$. With $r_{0}=\lambda_{0} / 1.5$ representing a significant surface curvature, the comparison is still close to perfect, which suggests that the GSTCs can be straight-forwardly applied to curvilinear surfaces. There is a slight discrepancy that gradually increases as curvature is made larger, although in all cases, a very good agreement still 


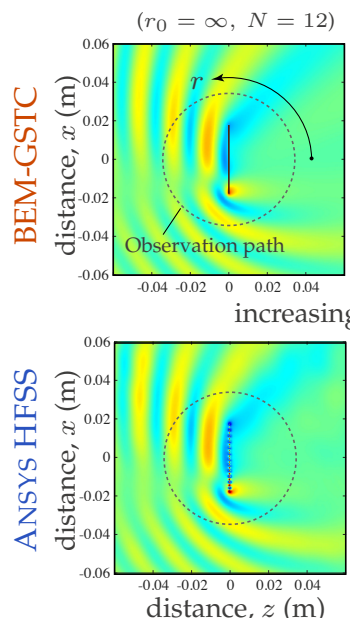

(a)
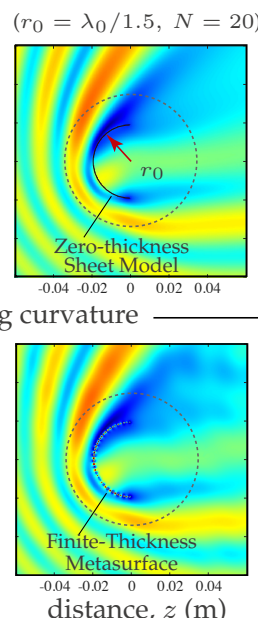

(b)

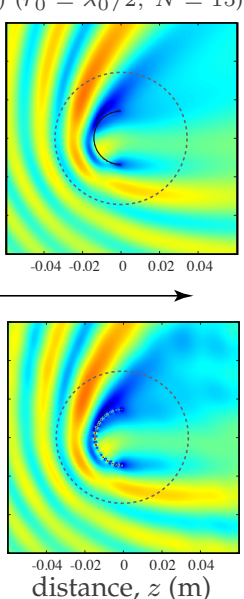

(c)

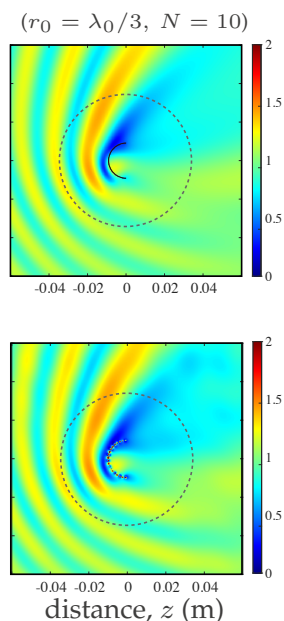

(d)

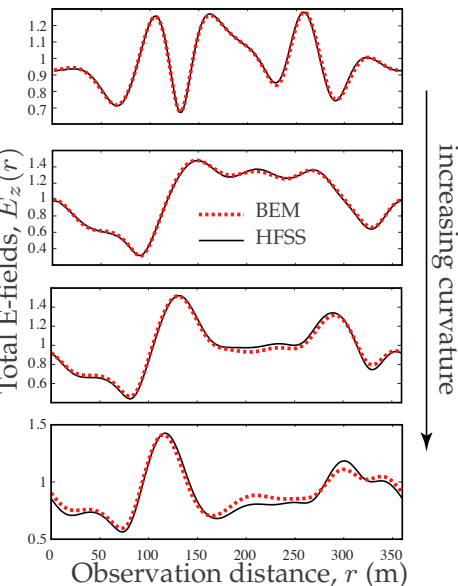

(e)

Figure 2.5 Comparison between the magnitude of the total E-fields calculated using BEM-GSTC and FEM-HFSS for a semi-circular metasurface as the curvature radius $r_{0}$ was decreased from $\infty$ (i.e. a flat surface), where $N$ is the number of cells. There is a plane wave incident at $\theta=30^{\circ}$ in the $x y$-plane, and the structure is uniform in the $z$ direction.

exists. To show this difference more clearly, the fields plotted along a circular observation line, are shown in Fig. 2.5e for various cases. I do note that for the last case, the surface is very small, with $r_{0}=\lambda_{0} / 3$ and only 10 cells. From this study, it is not clear whether the discrepancy is primarily due the diffraction around the ends of the finite surface, or due to the susceptibilities. I anticipate further studies in this direction.

\subsection{Conclusion}

The IBCs and GSTCs were presented as zero-thickness models for MSs, and the use of the latter was motivated by susceptibilities being true constitutive parameters, independent of the incident field. After simplifying the susceptibility tensors for a reciprocal and polarization-maintaining MS, equations were developed for predicting the S-parameters for plane wave excitation, and for extracting the susceptibilities. A simple example of modelling a curvilinear MS composed of metal loops was shown, with comparison to BEM showing that the susceptibilities provide an accurate compact model for full-wave simulations. This provides a starting point for performing susceptibility extractions in Chapter 3. 


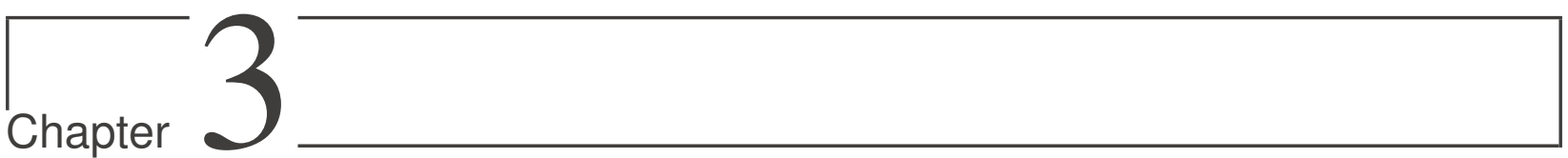

\section{SURFACE SUSCEPTIBILITY EXTRACTIONS OF CANONICAL STRUCTURES}

\subsection{Introduction}

In Section 2.4, a simple extraction was demonstrated, showing that surface susceptibilities are able to accurately model an single-layer array of resonators. ${ }^{1}$ This has been rigorously shown to be valid in numerous publications over the last two decades, e.g [9, 21, 10, 22, 23, 24, 13]. The mapping of susceptibilities for a single dielectric layer has also been shown [25, 26, 27]. On the other hand, one may wonder: is it possible to apply the GSTCs and the HK model to model a cascaded structure, such as dielectric layer, backed by a PEC ground-plane? While this has been considered using single-sided boundary conditions, which involve the fields which interact with the slab and reflect from the ground-plane [28, 29, 30], in this chapter I consider whether the two-sided GSTCs can be used to model such a structure. The model should have asymmetrical behaviour, behaving as a PEC with a cover layer on one side, and a PEC on the other side. The two sides are independent (electrically isolated), so it is not immediately clear that the HK model—which uses the average of the total fields as the acting fields-should work. Surprisingly, I will show that it is indeed possible with some limitations.

I will use (2.7) to determine the susceptibility models for several simple structures, progressively building up to a reflective metasurface, for which the susceptibilities correctly model the behaviour with illumination from either side. I follow a pedagogical approach, starting with simple structures such as a PEC sheet and a dielectric sheet to motivate the susceptibility terms in (2.5) and understand their role for the final reflective metasurface.

\footnotetext{
${ }^{1}$ While the loop cell in Figure 2.3a has two metal layers on either side of the substrate, in order to create the MIM capacitors, it is sufficiently thin with respect to the spacing between loops such that each loop structure behaves as a single resonator.
} 


\subsection{PEC Sheet}

Firstly, consider a perfect electric conductor (PEC) sheet, with $S_{\{21,12\}}^{\{\mathrm{TE}, \mathrm{TM}\}}=0$ and $S_{\{11,22\}}^{\{\mathrm{TE}, \mathrm{TM}\}}=-1$. Given the reflection symmetry, we must have $\chi_{\mathrm{em}}^{\{y x, x y\}}=0$. A PEC is the limiting case of a conductor with infinite conductivity $(\sigma \rightarrow \infty)$, which in fact, corresponds to a limiting case of $\chi_{\mathrm{ee}}^{x x}$ and $\chi_{\mathrm{ee}}^{y y}$. By eliminating all other susceptibility terms from (2.7) and taking a limit,

$$
\begin{gathered}
\lim _{\chi_{\mathrm{ee}}^{y y} \rightarrow \infty} S_{\{11,22\}}^{\mathrm{TE}}=\lim _{\chi_{\mathrm{ee}}^{y y} \rightarrow \infty}\left(\frac{k_{0}}{\frac{2 j \cos \theta}{\chi_{\mathrm{ee}}^{y y}}-k_{0}}\right)=-1 \\
\lim _{\chi_{\mathrm{ee}}^{y y} \rightarrow \infty} S_{\{21,12\}}^{\mathrm{TE}}=\lim _{\chi_{\mathrm{ee}}^{y y} \rightarrow \infty}\left(\frac{2 \cos \theta}{2 \cos \theta+j k_{0} \chi_{\mathrm{ee}}^{y y}}\right)=0 \\
\lim _{\chi_{\mathrm{ee}}^{x x} \rightarrow \infty} S_{\{11,22\}}^{\mathrm{TM}}=\lim _{\chi_{\mathrm{ee}}^{x x} \rightarrow \infty}\left(\frac{k_{0} \cos \theta}{\frac{2 j}{\chi_{\mathrm{ee}}^{x x}}-k_{0} \cos \theta}\right)=-1 \\
\lim _{\chi_{\mathrm{ee}}^{x x} \rightarrow \infty} S_{\{21,12\}}^{\mathrm{TM}}=\lim _{\chi_{\mathrm{ee}}^{x x} \rightarrow \infty}\left(\frac{2 j}{2 j-k_{0} \chi_{\mathrm{ee}}^{x x} \cos \theta}\right)=0
\end{gathered}
$$

Thus, $\chi_{\mathrm{ee}}^{\{x x, y y\}} \rightarrow \infty$ precisely models a PEC sheet. ${ }^{2}$ However, this is not suitable for numerical simulation, since it requires a limit.

Now, I consider if there is another susceptibility model, having finite susceptibilities, which could be used instead. To this end, I return to considering all 8 terms in (2.5). Of course, including the bianisotropic terms produces asymmetry with forwards/backwards illumination, and so here I seek possible compromise that may not rigorously model a PEC but is useful for numerical implementation. I will enforce $S_{11}^{\{\mathrm{TE}, \mathrm{TM}\}}(\theta)=-1$ and $S_{\{12,21\}}^{\{\mathrm{TE}, \mathrm{TM}\}}(\theta)=0$; these provide 6 equations from (2.7) since $S_{22}^{\{\mathrm{TE}, \mathrm{TM}\}}(\theta)$ is not enforced. After setting $\chi_{\{\mathrm{ee}, \mathrm{mm}\}}^{x x}=\chi_{\{\mathrm{ee}, \mathrm{mm}\}}^{y y}$ such that there is rotational symmetry around the $z$ axis, 6 unknown susceptibilities remain. Solving, the non-zero terms remaining are $\chi_{\mathrm{em}}^{y x}=+2 j / k_{0}$ and $\chi_{\mathrm{em}}^{x y}=-2 j / k_{0}$.

Now, what happens for backward illumination? Using $(2.7 \mathrm{a}, \mathrm{c})$ I find $S_{22}^{\{\mathrm{TE}, \mathrm{TM}\}}(\theta)=+1$. Thus, the model appears as a PEC with forward illumination and a PMC with backward illumination, independent of the angle of incidence. If $\chi_{\mathrm{em}}^{\{x y, y x\}}$ are negated, the structure is effectively reflected in the $x-y$ plane. The angular independence with these purely bi-anisotropic susceptibilities was studied in [31], where it was also noted that such a surface is only possible to fabricate with a physical unit cell at a single frequency, in the limit of zero loss. For this work, however, the inability to physically represent a PEC sheet (with correct behaviour on both sides) does not end up being critical in our our subsequent objective, when I later consider a dielectric cover layer added to the PMC side.

\footnotetext{
${ }^{2}$ Similarly, $\chi_{\mathrm{mm}}^{\{x x, y y\}} \rightarrow \infty$ would be a PMC.
} 


\subsection{Dielectric Sheet}

Building towards a resonator on top of a ground-plane with a cover layer, I next consider an isolated sheet of uniform permittivity $\epsilon_{r}=\left(\epsilon_{r}^{\prime}-j \epsilon_{r}^{\prime \prime}\right)$, having an imaginary part allowing for loss, with a thickness $d$. This mapping was considered in [27], where only the tangential \{ee, mm $\}$ susceptibilities were used and earlier in [26] where the normal components $\left(\chi_{\{\mathrm{ee}, \mathrm{mm}\}}^{z z}\right)$ were included. I will consider the extraction here for completeness, using a simple and accessible approach.

The analytical reflection and transmission through a dielectric slab, are well-known, given by

$$
\left[\begin{array}{c}
1 \\
S_{11}^{\mathrm{a}}(\theta)
\end{array}\right]=\underbrace{\frac{1}{\tau_{1}^{\mathrm{a}}(\theta)}\left[\begin{array}{cc}
1 & \rho_{1}^{\mathrm{a}}(\theta) \\
\rho_{1}^{\mathrm{a}}(\theta) & 1
\end{array}\right]}_{\text {air-dielectric interface }} \cdot \underbrace{\left[\begin{array}{cc}
e^{j \phi} & 0 \\
0 & e^{-j \phi}
\end{array}\right]}_{\begin{array}{c}
\text { propagation } \\
\text { in dielectric }
\end{array}} \cdot \underbrace{\frac{1}{\tau_{2}^{\mathrm{a}}(\theta)}\left[\begin{array}{cc}
1 & \rho_{2}^{\mathrm{a}}(\theta) \\
\rho_{2}^{\mathrm{a}}(\theta) & 1
\end{array}\right]}_{\text {dielectric-air interface }} \cdot\left[\begin{array}{c}
S_{21}^{\mathrm{a}}(\theta) \\
0
\end{array}\right]
$$

with $a \in\{\mathrm{TE}, \mathrm{TM}\}, \phi=k_{0} d \sqrt{\epsilon_{r}} \cos \theta$, and where $\rho_{\{1,2\}}^{\mathrm{a}}(\theta)$ and $\tau_{\{1,2\}}^{\mathrm{a}}(\theta)$ are the Fresnel coefficients for oblique incidence at the first and second interfaces, dependent on the polarization (TE/TM) [32]:

$$
\begin{aligned}
\tau_{1}^{\{\mathrm{TE}, \mathrm{TM}\}}(\theta) & =\frac{2 \eta_{\mathrm{T}, 1}^{\{\mathrm{TE}, \mathrm{TM}\}}(\theta)}{\eta_{\mathrm{T}, 0}^{\{\mathrm{TE}, \mathrm{TM}\}}(\theta)+\eta_{\mathrm{T}, 1}^{\{\mathrm{TE}, \mathrm{TM}\}}(\theta)} \\
\tau_{2}^{\{\mathrm{TE}, \mathrm{TM}\}}(\theta) & =\frac{2 \eta_{\mathrm{T}, 0}^{\{\mathrm{TE}, \mathrm{TM}\}}(\theta)}{\eta_{\mathrm{T}, 0}^{\{\mathrm{TE}, \mathrm{TM}\}}(\theta)+\eta_{\mathrm{T}, 1}^{\{\mathrm{TE}, \mathrm{TM}\}}(\theta)} \\
\rho_{1}^{\{\mathrm{TE}, \mathrm{TM}\}}(\theta) & =\frac{\eta_{\mathrm{T}, 1}^{\{\mathrm{TE}, \mathrm{TM}\}}(\theta)-\eta_{\mathrm{T}, 0}^{\{\mathrm{TE}, \mathrm{TM}\}}(\theta)}{\eta_{\mathrm{T}, 0}^{\{\mathrm{TE}, \mathrm{TM}\}}(\theta)+\eta_{\mathrm{T}, 1}^{\{\mathrm{TE}, \mathrm{TM}\}}(\theta)} \\
\rho_{1}^{\{\mathrm{TE}, \mathrm{TM}\}}(\theta) & =\frac{\eta_{\mathrm{T}, 0}^{\{\mathrm{TE}, \mathrm{TM}\}}(\theta)-\eta_{\mathrm{T}, 1}^{\{\mathrm{TE}, \mathrm{TM}\}}(\theta)}{\eta_{\mathrm{T}, 0}^{\{\mathrm{TE}, \mathrm{TM}\}}(\theta)+\eta_{\mathrm{T}, 1}^{\{\mathrm{TE}, \mathrm{TM}\}}(\theta)} \\
\eta_{\mathrm{T}, n}^{\{\mathrm{TE}, \mathrm{TM}\}}(\theta) & =\eta_{n}\left\{\begin{array}{l}
k_{n} / k_{z, n}, \text { for TE } \\
k_{z, n} / k_{n}, \text { for TM }
\end{array}\right. \\
k_{z, n} & =\sqrt{k_{n}^{2}-k_{0} \sin \theta}
\end{aligned}
$$

where $\eta_{n}=\eta_{0} / \sqrt{\epsilon_{r}}$ is the intrinsic impedance in the $n$th medium ( $n=0$ for air and $n=1$ for the dielectric), $\eta_{\mathrm{T}, n}$ is the transverse impedance, $k_{z, n}$ is the normal component of the wave vector, and $k_{n}=\sqrt{\epsilon_{r}} k_{0}$. The matrix equation (3.2) can be solved to provide a total for four expressions for $S_{\{11,21\}}^{\{\mathrm{TE}, \mathrm{TM}\}}(\theta)$.

With $\theta=0^{\circ}$, the normal susceptibility terms in (2.7f) are eliminated, and I set $\chi_{\mathrm{em}}^{\{y x, x y\}}=0$ so that there is symmetry between forwards and backwards illumination, I can substitute $S_{\{11,21\}}^{\text {TE,TM }\}}(0)$ 
from (3.2) into (2.7) to have four equations with the solution

$$
\begin{gathered}
\chi_{\mathrm{ee}}^{x x}=\chi_{\mathrm{ee}}^{y y}=\frac{2 \sqrt{\epsilon_{r}} \tan \left(\frac{k_{0} d \sqrt{\epsilon_{r}}}{2}\right)}{k_{0}} \\
\chi_{\mathrm{mm}}^{x x}=\chi_{\mathrm{mm}}^{y y}=\frac{2 \tan \left(\frac{k_{0} d \sqrt{\epsilon_{r}}}{2}\right)}{k_{0} \sqrt{\epsilon_{r}}}
\end{gathered}
$$

Repeating a similar procedure for $\theta \neq 0^{\circ}$, the normal susceptibility terms are found to be

$$
\begin{aligned}
\chi_{\mathrm{ee}}^{z z} & =\csc ^{2} \theta\left(\frac{2 \gamma \tan \left(\frac{k_{0} d \gamma}{2}\right)}{k_{0} \sqrt{\epsilon_{r}}}-\chi_{\mathrm{mm}}^{x x}\right) \\
\chi_{\mathrm{mm}}^{z z} & =\csc ^{2} \theta\left(\frac{2 \gamma \tan \left(\frac{k_{0} d \gamma}{2}\right)}{k_{0}}-\chi_{\mathrm{ee}}^{x x}\right)
\end{aligned}
$$

with $\gamma=\sqrt{\epsilon_{r}-\sin ^{2} \theta}$. As is, there is an angular dependence in (3.4c-d). This should not be the case, if $\chi_{\{\mathrm{ee}, \mathrm{mm}\}}^{z z}$ are to be true constitutive parameters, independent of the incident field [14]. However, I can only expect a zero-thickness model to apply for thin slabs $\left(k_{0} d \ll 1\right)$, and so I proceed by using the first few terms of the Taylor expansion around $k_{0} d=0$ :

$$
\begin{gathered}
\chi_{\mathrm{ee}}^{z z}=-\frac{d}{\epsilon_{r}}-\frac{k_{0}^{2} d^{3}}{6}+\frac{k_{0}^{2} d^{3} \sin ^{2} \theta^{-0}}{12 \epsilon_{r}}+O\left[\left(k_{0} d\right)^{4}\right] \\
\chi_{\mathrm{mm}}^{z z}=-d-\frac{k_{0}^{2} d^{3}}{6 \epsilon_{r}}+\frac{k_{0}^{2} d^{3} \sin ^{2} \theta^{-0}}{12}+O\left[\left(k_{0} d\right)^{4}\right]
\end{gathered}
$$

with the approximation that the third term and higher-order terms are negligible. Within this approximation, $\chi_{\{\mathrm{ee}, \mathrm{mm}\}}^{z z}$ only depends on the properties of the slab (at a given frequency), and can be considered constitutive parameters.

To validate the assumption and verify (3.4) and (3.5), I consider a numerical example, shown in Figure 3.1. The reflection and scattering for both TE and TM illuminations is shown, for increasing thickness $k_{0} d$ and at three angles, $\theta \in\left\{0^{\circ}, 30^{\circ}, 60^{\circ}\right\}$. Firstly, I see that even when the slab is very thin $\left(k_{0} d=0.2\right.$, i.e. $\left.d \approx \lambda / 30\right)$ the normal components $\chi_{\{\mathrm{ee}, \mathrm{mm}\}}^{z z}$ are needed to model the scattering at oblique incidence. Secondly, the modelling is very accurate up to about $k_{0} d=0.8(d \approx \lambda / 8)$, past which the analytical result diverges.

\subsection{PEC Sheet with Cover Layer}

Next, consider a PEC with a dielectric cover layer on one side. In this case, the forwards and backwards illuminations clearly have non-symmetrical behaviour. With the cover layer on the left 

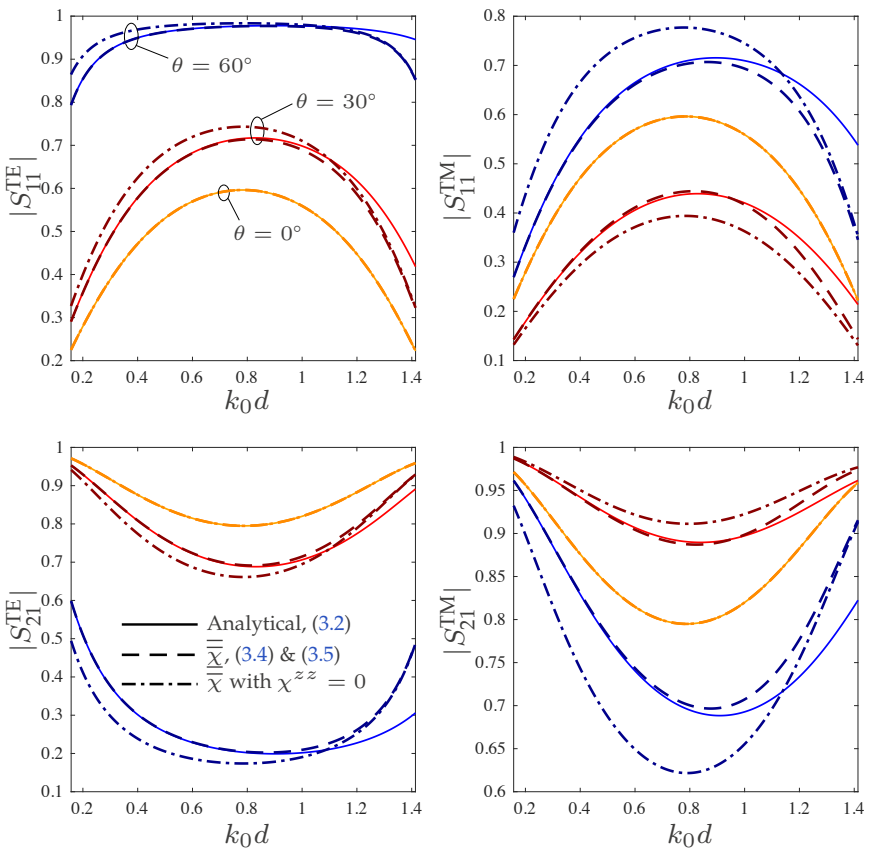

Figure 3.1 The reflection and transmission through a lossy dielectric sheet $\left(\epsilon_{r}=4-j 0.04\right)$ was calculated analytically using (3.2) to compare with the mapped susceptibilities (3.4), with and without the normal components.

side of the PEC sheet in Figure 2.2, the forwards illumination case is governed by

$$
\left[\begin{array}{c}
1 \\
S_{11}^{\mathrm{a}}(\theta)
\end{array}\right]=\underbrace{\frac{1}{\tau_{1}(\theta)}\left[\begin{array}{cc}
1 & \rho_{1}^{\mathrm{a}}(\theta) \\
\rho_{1}^{\mathrm{a}}(\theta) & 1
\end{array}\right]}_{\text {air-dielectric interface }} \cdot \underbrace{\left[\begin{array}{cc}
e^{j \phi} & 0 \\
0 & e^{-j \phi}
\end{array}\right]}_{\begin{array}{c}
\text { propagation } \\
\text { in dielectric }
\end{array}} \cdot\left[\begin{array}{c}
E_{\mathrm{PEC}}^{\mathrm{a}} \\
-E_{\mathrm{PEC}}^{\mathrm{a}}
\end{array}\right]
$$

where $E_{\mathrm{PEC}}^{\mathrm{a}}$ is the amplitude of the forwards-travelling wave at the PEC. Since $E_{\mathrm{PEC}}^{\mathrm{a}}$ is not of interest, this reduces to two expressions for $S_{11}^{\{\mathrm{TE}, \mathrm{TM}\}}(\theta)$, one for each polarization. Also, $S_{\{21,21\}}^{\{\mathrm{TE}, \mathrm{TM}\}}(\theta)=0$ and $S_{22}^{\{\mathrm{TE}, \mathrm{TM}\}}(0)=-1$. Substituting into (2.8),

$$
\begin{gathered}
\chi_{\mathrm{mm}}^{\{x x, y y\}}=0 \\
\chi_{\mathrm{em}}^{y x}=-\frac{2 j}{k_{0}} \\
\chi_{\mathrm{em}}^{x y}=+\frac{2 j}{k_{0}} \\
\chi_{\mathrm{ee}}^{\{x x, y y\}}=-\frac{4 \cot \left(k_{0} d \sqrt{\epsilon_{r}}\right)}{\sqrt{\epsilon_{r}}} \\
\chi_{\mathrm{ee}}^{z z} \approx-\frac{4 d}{\epsilon_{r}}-\frac{8 k^{2} d^{3}}{3} \\
\chi_{\mathrm{mm}}^{z z} \approx-\frac{4 d}{3}-\frac{8 k^{2} d^{3} \epsilon_{r}}{45}
\end{gathered}
$$



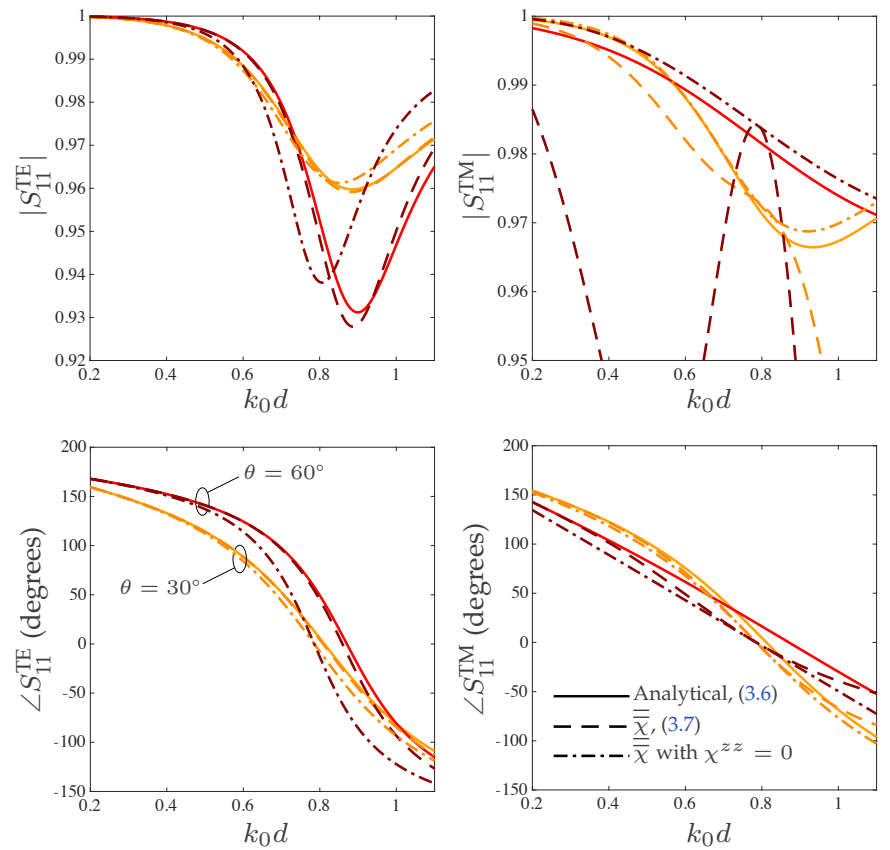

Figure 3.2 The reflection from a PEC sheet with a lossy dielectric cover layer $\left(\epsilon_{r}=4-j 0.04\right)$ was calculated analytically using (3.6) to compare with the mapped susceptibilities (3.6), with and without the normal components. Meanwhile, $S_{22}^{\{\mathrm{TE}, \mathrm{TM}\}}=-1$, and $S_{12,21}^{\{\mathrm{TE}, \mathrm{TM}\}}=0$ (not plotted).

after all but the leading angle-independent terms in the Taylor expansion are retained for (3.7e,f) (the complete expressions for $\chi_{\{\mathrm{ee}, \mathrm{mm}\}}^{z z}$ are cumbersome and not presented). It is interesting that $\chi_{\mathrm{em}}^{\{y x, x y\}}$ are in fact identical to those for a sheet that behaves as a PEC on the back side $\left(S_{22}^{\{\mathrm{TE}, \mathrm{TM}\}}=\right.$ $-1)$ and a PMC on the front side $\left(S_{11}^{\{\mathrm{TE}, \mathrm{TM}\}}=+1\right)$, as discussed in Section 3.2. However, in this case the other susceptibilities contribute such that the front does not appear as a PMC, but a PEC with a cover layer.

To check that this is is indeed the case, numerical results are shown in Figure 3.2. For TE polarization, (3.7) provides a good match for the forwards reflection past $k_{0} d>1$, in comparison to the analytical expression (3.6); the resonance at $k_{0} d \approx 0.9$ is correctly predicted. For TM polarization, there is reasonable agreement if $\chi_{\mathrm{ee}}^{z z}$ is neglected, but including causes a large disagreement. Thus, it seems that the truncation of the Taylor series in (3.7e) is a poor approximation, and this term should not be used. Meanwhile, $S_{\{21,12\}}^{\{\mathrm{TE}, \mathrm{TM}\}}=0$ and $S_{\{22\}}^{\{\mathrm{TE}, \mathrm{TM}\}}=-1$ (not plotted). That is, the model behaves precisely as a PEC with backwards illumination.

Now I consider: is (3.7) consistent for an incident plane wave propagating in the $y-z$ plane? The extraction for (3.7) [and (3.4) earlier] involved fields propagating in the $x-z$ plane, using (2.7). Meanwhile, a proper physical model of the surface should be accurate for an arbitrary incident field. Since the structures discussed thus far have rotational symmetry around the $z$ axis, the susceptibilities should remain unchanged with rotation about the $z$ axis. That is, $\overline{\bar{\chi}}=\overline{\bar{R}}_{z}(\phi) \cdot \overline{\bar{\chi}}$. 
$\overline{\bar{R}}_{z}(\phi)^{T}$ should hold true, where $\overline{\bar{R}}_{z}(\phi)$ is the transformation matrix which rotates by $\phi$ about the $z$ axis [15]. This is indeed the case, and so the susceptibilities presented work for an arbitrary incident field.

\subsection{Sub-wavelength Resonator on a Dielectric Slab}

Finally, I extend the structure by including an array of electric dipoles on top of the grounded slab; this can truly be called a metasurface. For simplicity, I will only consider TE polarization, since the unit cell was designed to resonate with TE polarization. Shown in Figure 3.3, the unit cell is deeply sub-wavelength $(\lambda / 10$ at $30 \mathrm{GHz})$. It has a "dogbone"-shaped copper dipole loaded with a lumped inductance $(L)$ at the center, placed on a Rogers RO4003C substrate ( $508 \mu \mathrm{m}$ thickness; i.e. $\left.k_{0} d=0.32\right)$, on top of a PEC ground-plane.

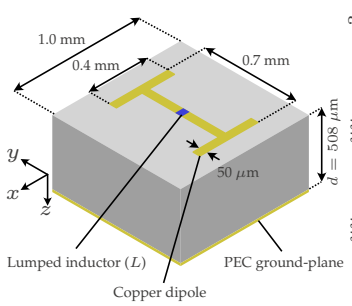

(a)

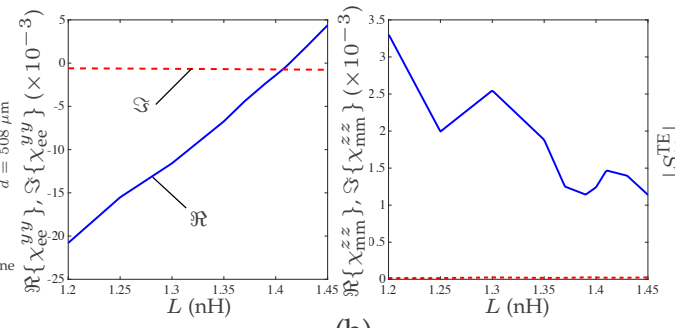

(b)

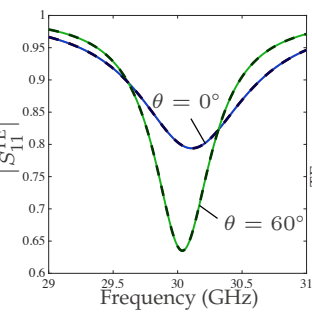

(c)

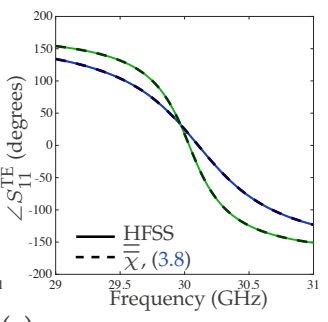

)

Figure 3.3 A deeply-subwavelength reflective unit cell was designed using an electric dipole, loaded with a lumped inductor $(L)$ on top of a Rogers RO4003C substrate $\left(\epsilon_{r}=3.55, \tan \delta_{d}=0.0027\right)$ that is on a PEC ground-plane. (a) Unit cell model. (b) $\overline{\bar{\chi}}$ extraction at $30 \mathrm{GHz}$ using (3.8), as a function of the loading inductance $L$. (c) $S_{11}^{\mathrm{TE}}$ with $L=1.4 \mathrm{nH}$ at two angles of incidence.

While there is no analytical expression for $S_{11}^{\mathrm{TE}}(\theta)$, it can be found through full-wave simulations of the unit cell $[13,12]$. In particular, two angles of incidence need to be simulated. Then, using (2.8) to solve the susceptibilities with $S_{\{21,21\}}^{\mathrm{TE}}(\theta)=0$ and $S_{22}^{\mathrm{TE}}(0)=-1$,

$$
\begin{gathered}
\chi_{\mathrm{mm}}^{x x}=0 \\
\chi_{\mathrm{em}}^{y x}=-\frac{2 j}{k_{0}} \\
\chi_{\mathrm{ee}}^{y y}=\frac{4 j\left(S_{11}^{\mathrm{TE}}(0)-1\right)}{k\left(S_{11}^{\mathrm{TE}}(0)+1\right)} \\
\chi_{\mathrm{mm}}^{z z}=\frac{4 j}{k} \csc \theta\left[\csc \theta\left(\frac{2}{S_{11}^{\mathrm{TE}}(0)+1}-1\right)+\cot \theta\left(1-\frac{2}{S_{11}^{\mathrm{TE}}(\theta)+1}\right)\right]
\end{gathered}
$$

with all other terms being irrelevant for TE polarization due to the lack of polarization conversion. This extraction was performed using full-wave simulations (Ansys HFSS) of the unit cell with periodic boundaries, using $\theta \in\left\{0^{\circ}, 60^{\circ}\right\}$, and with $1.2 \mathrm{nH} \leq L \leq 1.45 \mathrm{nH}$; the susceptibilities are

\footnotetext{
${ }^{3}$ This is similar to Section 3.4, but with the simulated S-parameters rather than analytical expressions
} 
plotted in Figure 3.3b. Both $\chi_{\mathrm{ee}}^{y y}$ and $\chi_{\mathrm{mm}}^{z z}$ are extracted, while $\chi_{\mathrm{em}}^{y x}$ only depends on frequency through (3.8b) (not plotted). I note that the inclusion of $\chi_{\mathrm{mm}}^{z z}$ has only a minor effect compared to $\chi_{\mathrm{ee}}^{y y}$, and so its profile in Fig $3.3 \mathrm{~b}$ is not smooth. Tightening the convergence criteria in HFSS and including more points for $L$ may help in a better extraction of $\chi_{\mathrm{mm}}^{z z}$. Nevertheless, the predicted reflection is plotted in Figure 3.3c for $L=1.4 \mathrm{nH}$, at which there is a resonance at $30 \mathrm{GHz}$. I observe nearly perfect agreement HFSS simulation for $S_{11}^{\mathrm{TE}}$ at the two different angles of incidence, while we also have $S_{22}^{\mathrm{TE}}=-1$ corresponding to an ideal PEC at all frequencies (not shown here) thanks to the inclusion of $\chi_{\mathrm{em}}^{y x}$. Thus, this combination of $\chi_{\mathrm{ee}}^{y y}, \chi_{\mathrm{em}}^{y x}$, and $\chi_{\mathrm{mm}}^{z z}$ produces a very accurate model for scattered TE-polarized fields, regardless of the incident field.

\subsection{Numerical Demontration: Parabolic Reflector}

Finally, I demonstrate the utility of the two-sided susceptibility model of the reflective unit cell from Section 3.5 by designing a parabolic reflector; i.e. a metasurface which reflects a plane wave when a cylindrical wave is present from a line source (forward illumination). The incident field is

$$
\begin{aligned}
& E_{\mathrm{i}, y}(\mathbf{r})=E_{0} \frac{H_{0}^{(2)}\left(k\left|\mathbf{r}-\mathbf{r}_{\mathrm{s}}\right|\right)}{H_{0}^{(2)}\left(k\left|\mathbf{r}_{\mathrm{s}}\right|\right)} \\
& H_{\mathrm{i}, x}(\mathbf{r})=E_{0} \frac{j\left(z-z_{\mathrm{s}}\right) H_{1}^{(2)}\left(k\left|\mathbf{r}-\mathbf{r}_{\mathrm{s}}\right|\right)}{\eta|\mathbf{r}| H_{0}^{(2)}\left(k\left|\mathbf{r}_{\mathrm{s}}\right|\right)} \\
& H_{\mathrm{i}, z}(\mathbf{r})=-E_{0} \frac{j\left(x-x_{\mathrm{s}}\right) H_{1}^{(2)}\left(k\left|\mathbf{r}-\mathbf{r}_{\mathrm{s}}\right|\right)}{\eta|\mathbf{r}| H_{0}^{(2)}\left(k\left|\mathbf{r}_{\mathrm{s}}\right|\right)}
\end{aligned}
$$

where $\mathbf{r}_{\mathrm{s}}=\left(x_{\mathrm{s}}, z_{\mathrm{s}}\right)$ is the location of the source, $H_{\{0,1\}}^{(2)}$ are Hankel functions of the second kind, and $E_{0}$ is the amplitude. For our example, $\mathbf{r}_{\mathrm{s}}=(0 \mathrm{~cm},-10 \mathrm{~cm})$, at $30 \mathrm{GHz}$. Meanwhile, the reflected field should have a uniform phase, while the amplitude is tapered so that the surface remains passive. Specifically,

$$
\begin{array}{r}
E_{\mathrm{r}, y}(x, 0)=0.9\left|E_{\mathrm{i}, y}(x, 0)\right| \\
H_{\mathrm{r}, x}(x, 0)=\frac{E_{\mathrm{r}, y}(x, 0)}{\eta}
\end{array}
$$

Here, the factor 0.9 was chosen arbitrarily to ensure passivity. Using (3.9) and (3.10), I calculate the ideal $\chi_{\text {ee }}^{y y}$ point-wise along $x$ using (3.8c), treating $\chi_{\mathrm{mm}}^{z z}$ as a perturbation. ${ }^{4}$ I do not aim to optimize the design, but rather wish to show how the two-sided susceptibility model is accurate for a finitesized and non-uniform surface. This produces the "desired" profile in Figure 3.4b. However, the unit cell extraction is limited in replicating this profile since there is only one parameter $L$, so that

\footnotetext{
${ }^{4}$ That is, I ignore $\chi_{\mathrm{mm}}^{z z}$ when designing the unit cell distribution, but will subsequently include it in the susceptibility model, for the prediction of fields.
} 
the real and imaginary parts of $\chi_{\mathrm{ee}}^{y y}$ cannot be tuned independently. For the sake of this demonstrate, the real part is realized, using the look-up plot from Figure 3.3b. Meanwhile, the $\Im\left(\chi_{\text {ee }}^{y y}\right)$ (and $\chi_{\mathrm{mm}}^{z z}$ ) are also included in the susceptibility model. As seen in Figure $3.4 \mathrm{~b}$, the imaginary part deviates from the desired profile. Finally, the profile is discretized into 50 cells, for a length of $50 \mathrm{~mm}$.

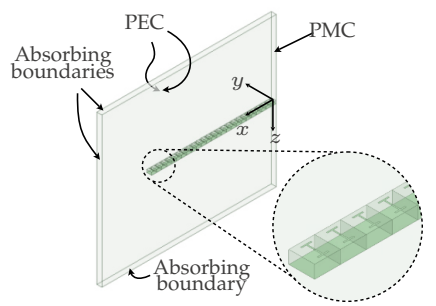

(a) HFSS Model

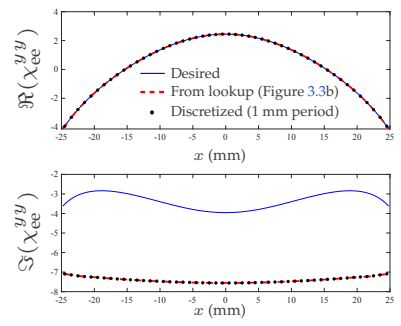

(b) $\chi_{\mathrm{ee}}^{y y}(x)$

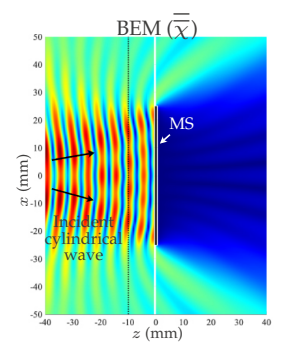

(c) $\left|\mathbf{E}_{\text {total }}(x, z)\right|$, Forwards illumination

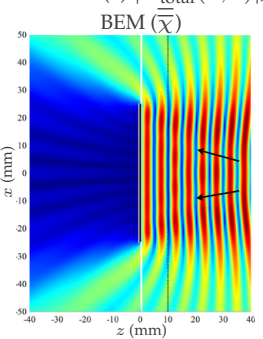

(e) $\left|\mathbf{E}_{\text {total }}(x, z)\right|$, Backwards illumination

HFSS
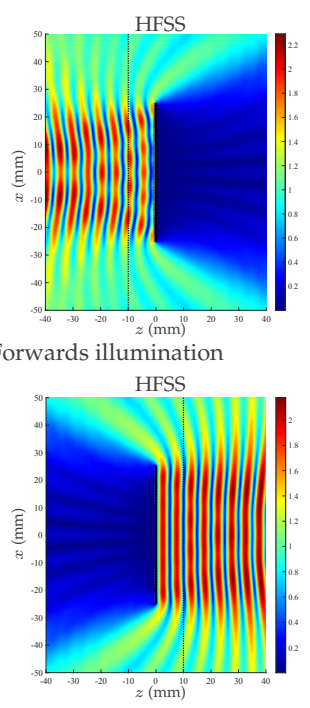

(f) $\mathbf{E}_{\mathrm{r}}(x, z)$ at $z=+10 \mathrm{~mm}$, backwards illumination

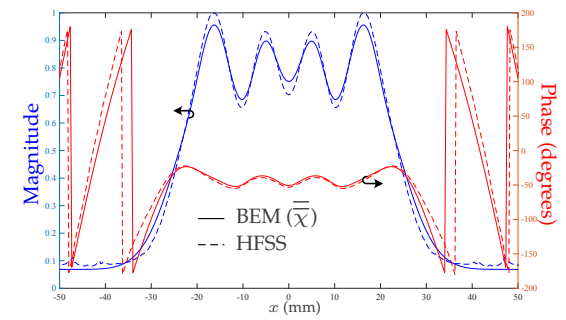

(d) $\mathbf{E}_{\mathrm{r}}(x, z)$ at $z=-10 \mathrm{~mm}$, forwards illumination

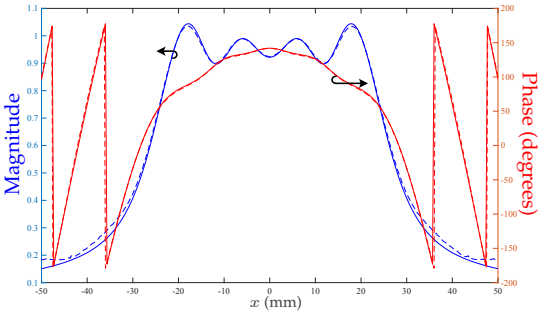

Figure 3.4 A parabolic reflector was designed using the unit cell from Figure 3.3, having a focal length of $10 \mathrm{~cm}$ with forwards illumination. (a) HFSS simulation model of half of the structure, with symmetry in the $y-z$ plane, and a total of $2 \times 25$ cells; i.e., total length of $5 \mathrm{~cm}$. (b) Desired and realized susceptibilities for reflecting a plane wave. (c) Comparison of the total field magnitude, using BEM (implementing the susceptibilities) and HFSS (full-wave), with an incident cylindrical wave originating from $\mathbf{r}_{\mathrm{s}}=(0 \mathrm{~cm},-10 \mathrm{~cm})$. (d) Comparison of reflected fields at $z=-10 \mathrm{~mm}$. (e-f) Same as (c-d) but with an incident cylindrical wave originating from $\mathbf{r}_{\mathrm{s}}=(0 \mathrm{~cm},+10 \mathrm{~cm})$

To check the accuracy of the susceptibility model for this finite non-uniform MS, I use an integralequation simulator which implements the complete dipolar GSTCs [18]. Meanwhile, the structure is simulated in HFSS using the model in Figure 3.4a, where I leverage symmetry in the $y-z$ plane to reduce the size of the model by half. To be precise, a PMC boundary at the $y-z$ plane effectively reflects the structure and fields across this plane. The magnitude of the total fields are compared in Figure 3.4c, for a cylindrical wave originating from $\mathbf{r}_{\mathrm{s}}=(0 \mathrm{~cm},-10 \mathrm{~cm})$, i.e., forwards illumination. We see a good match between the predicted total fields based on the non-uniform susceptibilities and the full-wave simulation, which takes orders of magnitude longer in time to simulate. Moreover, we see that the susceptibility model correctly models the diffraction around the edges of the finite surface, which is where "backward illumination" behavior is critical. The reflected fields at the $z=-10 \mathrm{~mm}$ plane are plotted for closer examination in Figure 3.4d. While an ideal parabolic reflector would produce a uniform amplitude and phase, there are fluctuations, which may be explained by the finite size of the reflector and edge diffraction, and the fact that the unit cell had only a single parameter of control so that only $\Re\left(\chi_{\mathrm{ee}}^{y y}\right)$ was implemented. However, it is not our purpose to optimize the design, which would require a more complex unit cell with 
loss, but rather to show the accuracy of the susceptibility model. To this end, we see an excellent match between BEM and HFSS.

Lastly to exemplify the two-sided GSTCs, in Figure 3.4e I repeat the simulations with a cylindrical wave originating from $\mathbf{r}_{\mathrm{s}}=(0 \mathrm{~cm}, 10 \mathrm{~cm})$, i.e., backward illumination. As expected, we observe a good match for the total fields, but the interference obscures the difference between the forwards and backwards illumination cases. By plotting the reflected fields alone (Figure 3.4e), we see that there is a curved phase, which is to be expected for a PEC sheet with an incident cylindrical wave. Thus, the PEC behavior with backwards illumination is correctly modeled and confirmed.

\subsection{Conclusions \& Future Work}

While it is not obvious a priori, I have shown that it is possible to use the conventional two-sided GSTCs and the HK model for the surface susceptibilities to predict the fields scattered from a fullyreflective metasurface. The susceptibilities work for both forwards and backwards illumination, behaving as a PEC for the latter, which is important if the surface is finite such that the fields may interact with the reverse side. This may be surprising, the HK model defines the acting fields as the average of the fields on either side, but the two sides are in fact electrically isolated and independent. I have also shown the mapping of the geometrical and electrical properties of a dielectric slab (and a PEC sheet with a dielectric cover layer) to susceptibilities, showing the role of the normal and bi-anisotropic terms. These equivalent surface susceptibilities thus act as compact models for these practically volumetric structures and be easily integrated into variety of simulation platforms to enable an efficient computation of scattered field from finite-sized volumetric structure for a faster iterative design flow.

As future work, the cell geometry could be made more complex, such as adding a normal polarizability (e.g. vertical dipole on a ground plane), or polarization conversion. Then, is it still possible to have a surface susceptibility representation that works for both forwards and backwards illumination? 


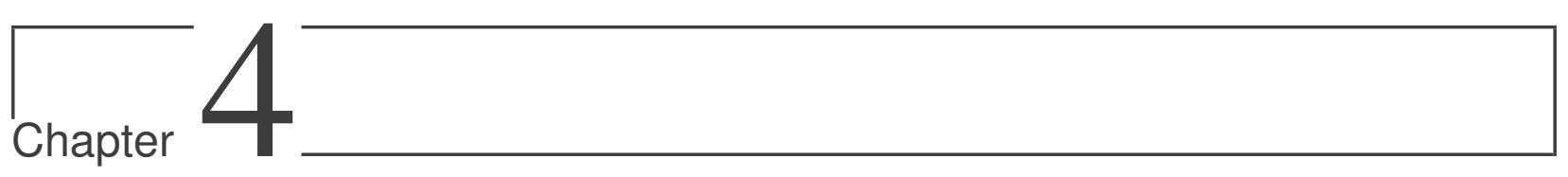

\section{FLOQUeT ANALYSis OF SPACE-Time Modulated Metasurfaces}

\subsection{Introduction}

Space-time modulated materials were studied in the context of parametric amplification [33, 34] in the 1950s, and have received renewed interest recently in the context of metamaterials. While static metamaterials have provided a plethora of wave manipulation devices [35, 36], they are typically limited by Lorentz reciprocity. While this can be overcome through the use of magneticoptic materials [37] or nonlinear materials [38], these methods require bulky implementations or provide weak non-reciprocity, respectively. For this reason, space-time modulated metamaterials have emerged as an appealing alternative: by modulating the constitutive parameters of a linear medium in space and time, it is possible to achieve strong reciprocity. This has been explored in 3-D bulk metamaterials [39, 40], 2-D MSs [41, 42, 43] and 1-D transmission lines [44, 45], with applications including isolators, circulators, and frequency mixers, and possibilities like using space-time diffraction patterns as channels for wireless communications [7] and non-reciprocal beam steering for full-duplex communications [8]. In experimental works, the surface properties have generally been manipulated using active components such as PIN diodes or varactors controlled by biasing circuitry with discrete states, which practically results in quantization error but nevertheless has demonstrated the practicality of the space-time approach $[6,46,47]$. Thus MSs, in particular, have recently gathered lot of attraction due to rich control over the scattered fields while offering practical simplicity. They are electromagnetically thin, consisting of a generally non-uniform array of sub-wavelength resonant particles.

To investigate into the properties of space-time modulated MSs, finite-difference time-domain (FDTD) techniques have recently been proposed to analyze a zero-thickness model of MSs [48, $49,50]$, based on the generalized sheet transition conditions (GSTCs) [9]. In this zero-thickness model, the resonant behavior of the scattering particles is described in terms of tensorial surface 
susceptibilities, $\overline{\bar{\chi}}$ accounting for both equivalent tangential and normal surface currents on the MS. Unlike frequency domain techniques that are typically used for static MSs like the finitedifference frequency-domain (FDFD) method [51] and the boundary element method (BEM) [52], FDTD lends itself naturally when the surface is time-varying, as demonstrated recently in [53]. However, if the modulation and incident field are periodic, then a steady-state will be achieved that is inefficient to compute with FDTD. In this case, an analytical approach would be desirable for efficiently computing the steady-state scattered fields; this can be done with the aid of Floquet's theorem which states that the fields in a periodic environment have a complex phase shift across each period [54]. ${ }^{1}$ Floquet's theorem can be used to show that the fields can be expressed in the form of (Floquet) harmonics.

Several analytical approaches have recently been presented to model general space-time varying electromagnetic systems. For instance, methods to compute fields scattered from bulk dielectric slabs whose permittivity $\epsilon_{\mathrm{r}}$ is periodically modulated in both space and time have been presented in [7] and [55]; these naturally involve a multiple interface problem, i.e. solving the Helmholtz wave equation. In these works, structures consist of dielectric interfaces only and no sub-wavelength resonating particles. While the explicit modelling of a finite thickness may seem to be a practical advantage, this is not appropriate for a MS where it has been shown that it is fundamentally incorrect and impractical to model a sheet of resonators with complex tensorial polarizabilities as an effective medium with a modulated effective permittivity and permeability $[10,27]$. Such a structure is meanwhile naturally treated with surface susceptibilities in a singleinterface problem using the GSTCs. If the structure is electromagnetically thin, the assumption of zero-thickness is a good approximation that yields a physical and tractable model that can take into account both normal and tangential surface polarization densities, along with bianisotropy. Consequently, calculations for a spatially-uniform time-varying MS are elegantly presented in [53], using a surface susceptibility model. It presents an analytical method to solve fields and polarization densities for the case of time modulation (1-D problem) while the general case of a 2D space-time varying surface is analyzed using FDTD. A more general treatment for space-time periodic surfaces was presented in [42], but this work was applicable for weak modulations. More recently, a method using a modulated electric surface conductivity was shown [56], but this does not allow for magnetic polarizability. In the same year, [41] presented another treatment using surface impedances, but these were taken to be frequency-independent, and thus do not take temporal dispersion into account. Moreover, none of these works explicitly considered the normal polarizabilities of the MSs, which are in general necessary to correctly model their angular scattering behavior.

Given MSs are constructed using sub-wavelength resonators which are inherently dispersive, their corresponding surface susceptibilities are naturally frequency-dependent. Moreover, quite often the surface is operated near resonance for maximal interaction of the waves with the surface. If the time modulation of the MS is slow compared to the incident field, it is reasonable to neglect

\footnotetext{
${ }^{1}$ For readers with a background in crystal structure, Bloch's theorem is mathematically the same [11].
} 
the frequency dependence, since the generated frequencies will be close to the incident frequency. However, for modulation frequencies that are not negligible compared to the excitation frequency, incorporating MS dispersion in the space-time analysis is important for obtaining correct field solutions. Keeping causality of the surface in mind, the frequency-dependent surface susceptibility distribution may not be arbitrarily applied and must be chosen with care.

In this chapter, I model the surface as having zero thickness and rigorously take into account the temporal dispersion using physically-motivated Lorentzian profiles to describe the susceptibilities (both tangential and normal). The Lorentzian parameters (e.g. resonant frequency) are parametrized to emulate a space-time modulation of the MS. Combined with GSTCs, the Floquet harmonic amplitudes are computed by solving a set of linear equations. The proposed semianalytical method thus efficiently computes the steady-state response of a general thin space-time modulated MS that is excited by a plane wave, whose surface susceptibilities are modulated with arbitrary periodicities in both space and time. This chapter focuses on the "diffraction regime" where the modulation is on a scale that is large enough to produce multiple propagating space and/or time harmonic plane waves (diffraction orders) ${ }^{2}$. I also show how the method can be extended to arbitrary excitations, such as Gaussian beams, by decomposing such fields into plane waves using Fourier decomposition. Furthermore, I demonstrate with a practical unit cell how a finite-thickness modulated MS is accurately and conveniently modelled with a zero-thickness sheet.

\subsection{Background}

Consider the problem in Fig. 4.1, where a MS placed at $z=0$ acts as a scatterer, producing reflected and transmitted fields, $\mathbf{E}_{\mathrm{r}}$ and $\mathbf{E}_{\mathrm{t}}$, when a incident field $\mathbf{E}_{\mathrm{i}}$ is present. ${ }^{3}$

\subsubsection{Linear Time Invariant (LTI) MSs}

If there is no time modulation, the constitutive relations can be written in the frequency domain as given in (2.4), but with the frequency and spatial dependence explicitly denoted here:

$$
\begin{aligned}
& \mathbf{P}(x, \omega)=\epsilon_{0} \overline{\bar{\chi}}_{\mathrm{ee}}(x, \omega) \mathbf{E}_{\mathrm{av}}(x, \omega)+\overline{\bar{\chi}}_{\mathrm{em}}(x, \omega) \sqrt{\mu_{0} \epsilon_{0}} \mathbf{H}_{\mathrm{av}}(x, \omega), \\
& \mathbf{M}(x, \omega)=\overline{\bar{\chi}}_{\mathrm{mm}}(x, \omega) \mathbf{H}_{\mathrm{av}}(x, \omega)+\overline{\bar{\chi}}_{\mathrm{me}}(x, \omega) \sqrt{\frac{\epsilon_{0}}{\mu_{0}}} \mathbf{E}_{\mathrm{av}}(x, \omega),
\end{aligned}
$$

The component of the susceptibility tensors $\overline{\bar{\chi}}$ normal to the surface are often zero or negligible. $[57,58]$, and are often discarded, for simplicity [14], especially in cases of field propagation normal to the surface. However, this is not the case in general when oblique wave incidence and

\footnotetext{
${ }^{2}$ Explicitly, I consider spatial modulations with a period greater than a wavelength and temporal modulations with a lower frequency than the incident field.

${ }^{3}$ This is the "forwards illumination" case from Figure 2.2.
} 


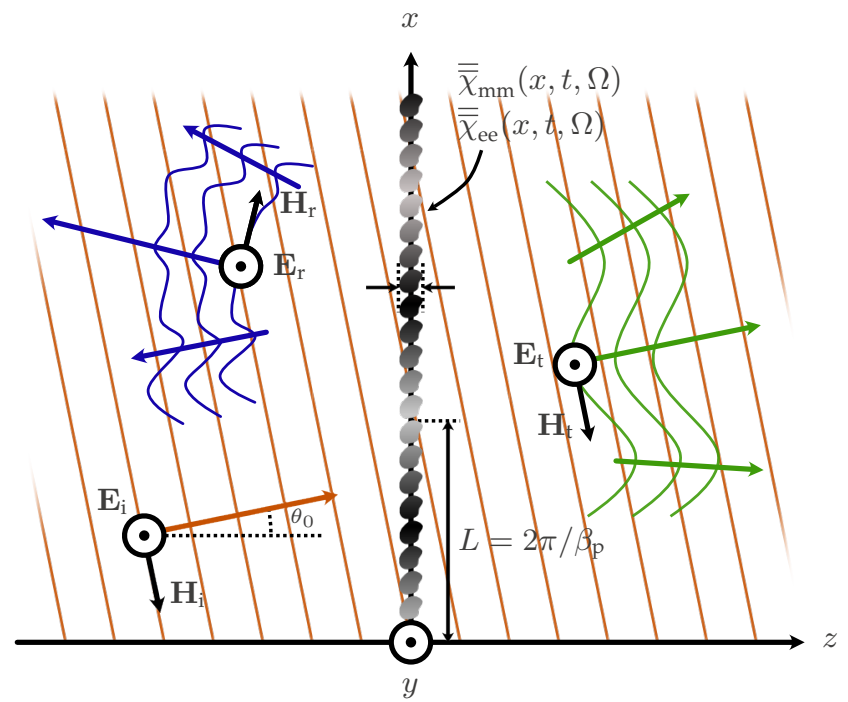

Figure 4.1 An illustration of scattering from from a space-time modulated MS with a spatial period $L$, where a TE plane wave incident at an angle $\theta_{0}$ produces reflected and transmitted fields. Surface susceptibilities model the structure as having zero thickness $d$, while the surface extends infinitely along $x$ and $y$.

general angular field scattering is considered. In this work, I will consider TE illumination as in Fig. 4.1, and include the normal susceptibility $\chi_{\mathrm{mm}}^{z z}$ in addition to $\chi_{\mathrm{mm}}^{y y}$ and $\chi_{\mathrm{ee}}^{x x}$, to demonstrate the generality of this approach. These terms are selected as they are the only ones involved for a reciprocal, polarization-maintaining MS with TE incidence in the $x z$-plane; this includes many practical surfaces of interest. The method is scalable to include other terms, such as those resulting in bianisotropy, $\overline{\bar{\chi}}_{\mathrm{em}}$ and $\overline{\bar{\chi}}_{\mathrm{me}}$. In the aforementioned TE case, after eliminating the bianisotropic terms in (4.1) it can be written in the time domain as $[59,60,61]$

$$
\begin{gathered}
Q^{y}(x, t)=\int_{-\infty}^{\infty} \chi_{\mathrm{ee}}^{y y}(x, \tau) E_{\mathrm{av}}^{y}(x, t-\tau) d \tau \\
M^{\{x, z\}}(x, t)=\int_{-\infty}^{\infty} \chi_{\mathrm{mm}}^{\{x x, z z\}}(x, \tau) H_{\mathrm{av}}^{\{x, z\}}(x, t-\tau) d \tau
\end{gathered}
$$

where the electric polarization has been normalized so $Q^{y}=P^{y} / \epsilon_{0}$, for a more symmetric formulation. The averages of the fields at $z=0$ are

$$
\begin{aligned}
E_{\mathrm{av}}^{y} & =\frac{E_{\mathrm{i}}^{y}+E_{\mathrm{r}}^{y}+E_{\mathrm{t}}^{y}}{2}, \\
H_{\mathrm{av}}^{\{x, z\}} & =\frac{H_{\mathrm{i}}^{\{x, z\}}+H_{\mathrm{r}}^{\{x, z\}}+H_{\mathrm{t}}^{\{x, z\}}}{2} .
\end{aligned}
$$


In the time domain, the GSTCs from (2.3) become

$$
\begin{aligned}
\hat{\mathbf{z}} \times \Delta \mathbf{H}(x, t) & =\epsilon_{0} \frac{d \mathbf{Q}^{\|}(x, t)}{d t}-\hat{\mathbf{z}} \times \nabla M^{z}, \\
\Delta \mathbf{E}(x, t) \times \hat{\mathbf{z}} & =\mu_{0} \frac{d \mathbf{M}^{\|}(x, t)}{d t}-\nabla Q^{z} \times \hat{\mathbf{z}},
\end{aligned}
$$

which in the TE case simplify to

$$
\begin{aligned}
E_{\mathrm{t}}^{y}-\left(E_{\mathrm{r}}^{y}+E_{\mathrm{i}}^{y}\right) & =\mu_{0} \frac{d M^{x}(x, t)}{d t}, \\
H_{\mathrm{t}}^{x}-\left(H_{\mathrm{r}}^{x}+E_{\mathrm{i}}^{x}\right) & =\epsilon_{0} \frac{d Q^{y}(x, t)}{d t}-\frac{d M^{z}(x, t)}{d x} .
\end{aligned}
$$

Together, (4.2) and (4.5) are sufficient to uniquely solve for the fields and polarizations, given the susceptibilities and an incident field. The interest is in solving this problem, in the case that $\chi_{\mathrm{ee}}^{y y}$ and $\chi_{\mathrm{mm}}^{\{x x, z z\}}$ are modulated in time in addition to space. Specifically, I will consider the form of the fields when the spatial variation is periodic (with spatial angular frequency $\beta_{\mathrm{p}}=2 \pi / L$ as in Fig. 4.1) and the time variation is periodic (with "pumping" frequency $\omega_{p}=2 \pi / T$ ). The resulting fields will be described as a summation of infinite space-time harmonics, which I wish to determine, assuming a temporally dispersive MS.

\subsubsection{Linear Time-Variant (LTV) MSs}

I now consider how (4.2) generalizes for time-dependent susceptibilities. This convolution can be viewed as an input (field)-output (polarization) system, which is linear and time-invariant (LTI). A time-varying susceptibility on the other hand represents a linear time-variant (LTV) system, where the electric polarization density as an example can be written [61,62]

$$
Q^{y}(x, t)=\int_{-\infty}^{\infty} \chi_{\mathrm{ee}}^{y y}(x, t, \tau) E_{\mathrm{av}}^{y}(x, t-\tau) d \tau \text {. }
$$

This is a generalized convolution, where the impulse response $\chi_{\mathrm{ee}}^{y y}(x, t, \tau)$ gives the polarization observed at time $t$ due to an input field applied $\tau$ time units earlier (i.e. at $t-\tau$ ). ${ }^{4}$ It is worth emphasizing that although there is time variation, this should not be confused with a nonlinear system; $\chi_{\mathrm{ee}}^{y y}$ does not depend on the magnitude of $E_{\mathrm{av}}^{y}$.

In previous works where temporal dispersion is neglected, this implicitly means $\chi_{\mathrm{ee}}^{y y}(x, t, \tau)=$ $\chi_{\mathrm{ee}}^{y y}(x, t) \delta(t-\tau)$. With such an assumption, and furthermore specifically for periodic modulations,

\footnotetext{
${ }^{4}$ The system satisfies causality if $\chi_{\mathrm{ee}}^{y y}=0$ for $\tau<0$; that is, there cannot be a response before the excitation. The same applies for (4.2). If the system is causal, the integration limits simplify to $\int_{0}^{\infty} d \tau$ [60].
} 
the susceptibilities can be expressed as a Fourier series:

$$
\chi_{\{\mathrm{ee}, \mathrm{mm}\}}^{\{x x, z y\}}(x, t)=\sum_{r=-\infty}^{\infty} \sum_{s=-\infty}^{\infty} \tilde{\chi}_{\{\mathrm{ee}, \mathrm{mm}\}}^{\{x x, y y, z z\}}(r, s) e^{j\left(s \omega_{\mathrm{p}} t-r \beta_{\mathrm{p}} x\right)}
$$

with a tilde added to denote the frequency domain. This "temporally dispersionless" assumption may be acceptable when the static (non-modulated) susceptibility is a weak function of frequency, but this is not always the case. To consider more carefully, I use the Fourier transform of $\chi_{\mathrm{ee}}^{y y}(x, t, \tau)$ with respect to $\tau$, so that (4.6) can be written for an arbitrary signal $E_{\mathrm{av}}^{y}$ in an equivalent relation $[61,63]$

$$
Q^{y}(x, t)=\frac{1}{2 \pi} \int_{-\infty}^{\infty} \chi_{\mathrm{ee}}^{y y}(x, t, \Omega) E_{\mathrm{av}}^{y}(x, \Omega) e^{j \Omega t} d \Omega
$$

If the dependence of $\chi_{\mathrm{ee}}^{y y}$ on $\Omega$ is weak, such as when operating far from any resonances, then it can be safe to assume $\chi_{\mathrm{ee}}^{y y}(x, t, \Omega)=\chi_{\mathrm{ee}}^{y y}(x, t)$, or equivalently, $\chi_{\mathrm{ee}}^{y y}(x, t, \tau)=\chi_{\mathrm{ee}}^{y y}(x, t) \delta(t-\tau)$. Alternatively, I observe that if the surface is time-invariant (no dependency on $t$ ), then neglecting the temporal dispersion is reasonable if $E_{\mathrm{av}}^{y}(x, \Omega)$ is monochromatic. However, even with a monochromatic incident field $E_{\mathrm{i}}^{y}$, introducing time-dependence of the susceptibility will result in $Q^{y}(x, t)$ that is not monochromatic, and subsequently fields that are not monochromatic via (4.5). Thus, the frequency dispersion inherent to a static MS must in general be considered when time modulation is added, this being particularly critical near to resonances of the static susceptibility.

\subsubsection{Lorentzian Susceptibilities}

To model the temporal dispersion inherent to a static MS, a Lorentzian distribution provides a physically-motivated response that can be used to model MSs [48][54, pp. 317-318]. In the timedomain, this is a damped oscillator model that can be expressed as

$$
\begin{gathered}
\left(\left[\zeta_{\mathrm{ee}}^{y y}(x, t)\right]^{2}+\frac{d^{2}}{d t^{2}}+\alpha_{\mathrm{ee}}^{y y}(x, t) \frac{d}{d t}\right) Q^{y}(x, t)=\left[\xi_{\mathrm{ee}}^{y y}(x, t)\right]^{2} E_{\mathrm{av}}^{y}(x, t), \\
\left(\left[\zeta_{\mathrm{mm}}^{\{x x, z z\}}(x, t)\right]^{2}+\frac{d^{2}}{d t^{2}}+\alpha_{\mathrm{mm}}^{\{x x, z z\}}(x, t) \frac{d}{d t}\right) M^{\{x, z\}}(x, t)=\left[\xi_{\mathrm{mm}}^{\{x x, z z\}}(x, t)\right]^{2} H_{\mathrm{av}}^{\{x, z\}}(x, t),
\end{gathered}
$$

where $\zeta$ is the resonant frequency of the oscillator, $\alpha$ corresponds to damping (loss), and $\xi$ is the plasma frequency, where their explicit space-time dependence is included, for generality. Notice that the resonator is driven by the average fields at the surface (4.3) as in the constitutive relation (4.6). I could solve the differential equations (4.9) to obtain the impulse response for use in (4.6) [63]. However, I will use (4.9) directly, which takes into account the temporally dispersive nature of the surface [39]. 
As mentioned above, all six parameters in (4.9) are time-variant in general for a time-varying surface. Since the Lorentzian parameters are periodic in space and time, they can be written as Fourier series'. ${ }^{5}$ Using $\chi_{\mathrm{ee}}^{y y}$ as an example,

$$
\left\{\left[\zeta_{\mathrm{ee}}^{y y}(x, t)\right]^{2}, \alpha_{\mathrm{ee}}^{y y}(x, t),\left[\xi_{\mathrm{ee}}^{y y}(x, t)\right]^{2}\right\}=\sum_{r=-\infty}^{\infty} \sum_{s=-\infty}^{\infty}\left\{\tilde{\zeta}_{\mathrm{ee}}^{y y}(r, s), \tilde{\alpha}_{\mathrm{ee}}^{y y}(r, s), \tilde{\xi}_{\mathrm{ee}}^{y y}(r, s)\right\} e^{j\left(s \omega_{\mathrm{p}} t-r \beta_{\mathrm{p}} x\right)}
$$

Note that in general, several resonators governed by (4.9) may be required to have an accurate model of the MS, in which case responses of each of the resonators can be summed following the superposition principle, as the system is linear (e.g. $M^{x}=M_{1}^{x}+M_{2}^{x}+\cdots$ where each $M_{n}^{x}$ is due to a resonator with unique parameters given by (4.10)). Also note that while I consider TE fields in our analysis to demonstrate the method, for conciseness and simplicity, it can be straightforwardly extended to TM fields as well.

\subsection{Harmonic Expansion of Equations and Fields}

As alluded, I express the fields as

$$
\mathbf{E}_{\mathrm{a}}(x, z, t)=\hat{\mathbf{y}} \sum_{m=-\infty}^{\infty} \sum_{n=-\infty}^{\infty} \tilde{E}_{\mathrm{a}}(m, n) e^{j \Theta_{m n}} e^{ \pm j k_{z}(m, n) z},
$$

where $\mathrm{a}=(\mathrm{i}, \mathrm{r}, \mathrm{t})$ for the incident, reflected, and transmitted fields, ${ }^{6}$ respectively, and where $\Theta_{m n}=\left[\omega(n) t-k_{x}(m) x\right]$. Only a single harmonic is present for the incident field, $(m, n)=\left(m_{\mathrm{i}}, n_{\mathrm{i}}\right)$, corresponding to a plane wave.

Floquet's theorem prescribes that the transverse part of the wavevector $\left(k_{x}\right)$ takes on discrete values determined by spatial periodicity, and the normal component $\left(k_{z}\right)$ then follows from having a total magnitude $k(n)$ :

$$
\begin{gathered}
k_{x}(m)=k_{0} \sin \theta_{0}+m \beta_{\mathrm{p}}, m \in \text { integers } \\
k_{z}(m, n)=\sqrt{k^{2}(n)-k_{x}^{2}(m, n)},
\end{gathered}
$$

This idea can be extended for the time harmonics, where the frequency can also only change by multiples of the pumping frequency, and the wavenumber changes accordingly:

$$
\begin{gathered}
\omega(n)=\omega_{0}+n \omega_{\mathrm{p}}, n \in \text { integers, } \\
k(n)=k_{0}+n \frac{\omega_{\mathrm{p}}}{c_{0}}=k_{0}\left(1+n \frac{\omega_{\mathrm{p}}}{\omega_{0}}\right) .
\end{gathered}
$$

\footnotetext{
${ }^{5}$ It is important to note that the squares of the resonant and plasma frequencies in (4.9) are Fourier-decomposed.

${ }^{6}$ The sign on $\pm j k_{z, m n} z$ is $(-)$ for incident and transmitted harmonics and $(+)$ for reflected harmonics.
} 
Note that this allows for harmonics with negative frequencies, as well as potentially a dc "harmonic" if $\omega_{0}$ is an integer multiple of $\omega_{\mathrm{p}}$. The harmonics with $\omega(n)<0$ are not a cause for alarm, as $k(n)<0$ for these harmonics and so the direction of propagation is physical (e.g. still $+z$ in the transmission region). The special case of $\omega(n)=0$ is more questionable, but in the results, I show that this peculiarity also poses no problem.

The angles of scattering for the harmonics are found from $\sin [\theta(m, n)]=k_{x}(m) / k(n)$, using (4.12a) and (4.12d), which yields

$$
\sin [\theta(m, n)]=\left(\frac{\sin \theta_{0}+m \beta_{\mathrm{p}} / k_{0}}{1+n \omega_{\mathrm{p}} / \omega_{0}}\right) .
$$

where $\theta_{0}=\theta(0,0)$ is the angle of the fundamental harmonic. Each harmonic $(m, n)$ represents either an oblique propagating plane wave $\left(k_{z} \in \mathbb{R}\right)$ or a surface wave $\left(k_{z} \in \mathbb{I}\right)$, as illustrated in Fig. 4.2, where the real parts of the corresponding wavevectors are plotted. Using (4.12d), I plot circles with constant $k(n)$ (and $\omega(n)$ ), while (4.12a) yields the horizontal lines that represent the allowed values $k_{x}(m)$ (which is always purely real). The intersection of the circles and lines represents possible propagating space-time harmonics, also called diffraction orders. Surface waves on the other hand lie on the vertical axis and not necessarily on a $k(n)$ circle.

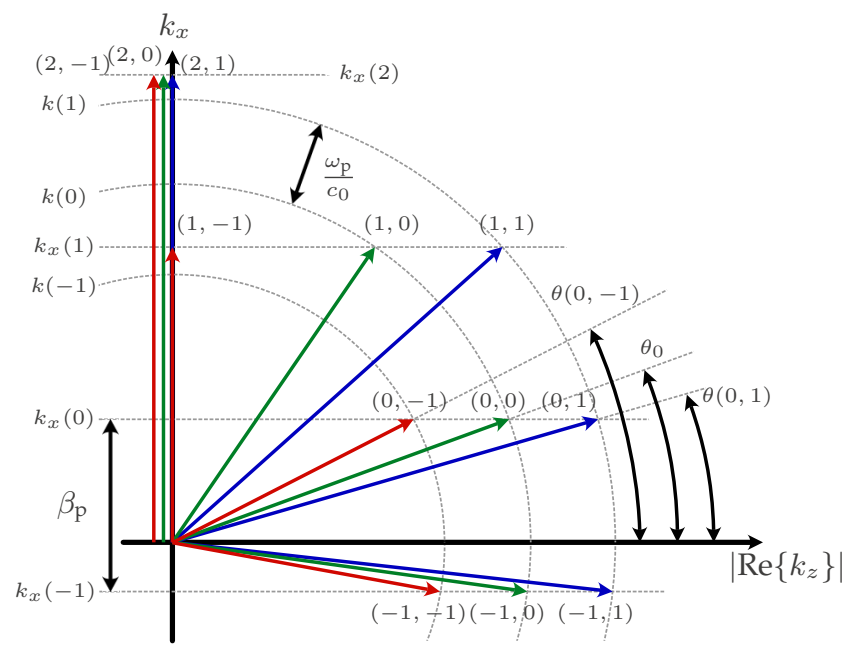

Figure 4.2 Space-time modulation produces scattered harmonics $(m, n)$ that exist at discrete frequencies $\omega(n)$ (hence the $k(n)$ circles) and discrete transverse spatial frequencies $k_{x}(m)$ (horizontal lines). At each $k_{x}(m)$, there are an infinite number of time harmonics with different $k(n)$ and thus different angles of scattering $\theta(m, n)$. This figure may be seen as a limiting case of Fig. 3 in [7], where $d \rightarrow 0$.

Finally, the magnetic field can also be expanded,

$$
\mathbf{H}_{a}(x, z, t)=\frac{1}{\eta_{0}} \sum_{m=-\infty}^{\infty} \sum_{n=-\infty}^{\infty}\left[\begin{array}{l}
\left(\frac{k_{x}(m)}{k(n)} \hat{\mathbf{z}} \pm \frac{k_{z}(m, n)}{k(n)} \hat{\mathbf{x}}\right) \\
\times \tilde{E}_{a}(m, n) e^{j \Theta_{m n}} e^{ \pm j k_{z}(m, n) z}
\end{array}\right],
$$


along with the polarization densities, which are

$$
\begin{aligned}
Q^{y}(x, t) & =\sum_{m=-\infty}^{\infty} \sum_{n=-\infty}^{\infty} \tilde{Q}^{y}(m, n) e^{j \Theta_{m n}}, \\
M^{\{x, z\}}(x, t) & =\sum_{m=-\infty}^{\infty} \sum_{n=-\infty}^{\infty} \tilde{M}^{\{x, z\}}(m, n) e^{j \Theta_{m n}} .
\end{aligned}
$$

\subsection{Matrix Formulation}

There are five sets of unknown harmonic amplitudes, $\tilde{E}_{\mathrm{r}}, \tilde{E}_{\mathrm{t}}, \tilde{Q}^{y}, \tilde{M}^{x}$, and $\tilde{M}^{z}$, for the TE illumination in consideration. To write out the formulation compactly and for ease of software implementation, each of these is written as an (initially infinite) column vector using a bijective (one-to-one) mapping $q \mapsto[m(q), n(q)]$. That is, each value of $q$ corresponds to a unique $(m, n)$ harmonic, with transmission coefficients from (4.11) written

$$
\tilde{\mathbf{E}}_{\mathrm{t}}=\left[\cdots, \tilde{E}_{\mathrm{t}}(q-1), \tilde{E}_{\mathrm{t}}\left(q_{0}\right), \tilde{E}_{\mathrm{t}}\left(q_{1}\right), \cdots\right]^{T}
$$

and likewise for $\tilde{\mathbf{E}}_{\mathrm{r}}, \tilde{\mathbf{Q}}_{y}, \tilde{\mathbf{M}}_{x}$, and $\tilde{\mathbf{M}}_{z}$. For example, consider a simple case with with $M=1$ and $N=1$. Then, the spatial indices are $m \in[-1,0,1]$ while the temporal indices are $n \in[-1,0,1]$, for a total of 9 space-time harmonics. Note that this few harmonics would generally be insufficient for an accurate solution, but is selected merely to show the matrices explicitly. The mapping $q \mapsto$ $[m(q), n(q)]$ is arbitrary but needs to be bijective. For example, one could use Table 4.1. For this example, each of the five column vectors (4.16) of unknown field and polarization densities has 9 entries.

Table 4.1 Example $q \mapsto[m(q), n(q)]$ Mapping

\begin{tabular}{cccccccccc}
\hline$q$ & 0 & 1 & 2 & 3 & 4 & 5 & 6 & 7 & 8 \\
\hline$m(q)$ & -1 & -1 & -1 & 0 & 0 & 0 & 1 & 1 & 1 \\
$n(q)$ & -1 & 0 & 1 & -1 & 0 & 1 & -1 & 0 & 1 \\
\hline
\end{tabular}

\subsubsection{Lorentzian Formulation}

I define matrices with the diagonal terms given by $\mathbb{\Omega}(q, q)=\omega(n(q))$, and $\mathbb{K}(q, q)=k(n(q))$, $\mathbb{K}_{x}(q, q)=k_{x}(m(q))$, and $\mathbb{K}_{z}(q, q)=k_{z}(m(q), n(q))$. Now substituting the field expansions (4.11), 
(4.14), and (4.15) into the constitutive relations (4.9), I find three sets of equations ${ }^{7}$

$$
\begin{gathered}
\left(\mathbb{Z}_{\mathrm{e}}^{y}-\Omega^{\odot 2}+j \mathbb{A}_{\mathrm{e}}^{y} \Omega\right) \tilde{\mathbf{Q}}_{y}=\frac{1}{2} \mathbb{X}_{\mathrm{e}}^{y}\left(\tilde{\mathbf{E}}_{\mathrm{r}}+\tilde{\mathbf{E}}_{\mathrm{t}}+\tilde{\mathbf{E}}_{\mathrm{i}}\right) \\
\left(\mathbb{Z}_{\mathrm{m}}^{x}-\Omega^{\odot 2}+j \mathbb{A}_{\mathrm{m}}^{x} \Omega\right) \tilde{\mathbf{M}}_{x}=\frac{1}{2 \eta_{0}} \mathbb{X}_{\mathrm{m}}^{x}\left(\mathbb{K}_{z} \oslash \mathbb{K}\right)\left(\tilde{\mathbf{E}}_{\mathrm{r}}-\tilde{\mathbf{E}}_{\mathrm{t}}-\tilde{\mathbf{E}}_{\mathrm{i}}\right) \\
\left(\mathbb{Z}_{\mathrm{m}}^{z}-\Omega^{\odot 2}+j \mathbb{A}_{\mathrm{m}}^{z} \Omega\right) \tilde{\mathbf{M}}_{z}=\frac{1}{2 \eta_{0}} \mathbb{X}_{\mathrm{m}}^{z}\left(\mathbb{K}_{x} \oslash \mathbb{K}\right)\left(\tilde{\mathbf{E}}_{\mathrm{r}}+\tilde{\mathbf{E}}_{\mathrm{t}}+\tilde{\mathbf{E}}_{\mathrm{i}}\right)
\end{gathered}
$$

where $\tilde{\mathbf{E}}_{\mathrm{i}}$ contains zeros expect for the incident harmonic $\tilde{E}_{\mathrm{i}}\left(m_{\mathrm{i}}, n_{\mathrm{i}}\right)=E_{0}$ (in our results, I use $E_{0}=1 \mathrm{~V} / \mathrm{m}$ ). The matrices $\mathbb{Z}, \mathbb{A}$, and $\mathbb{X}$ depend on the Lorentzian parameters from (4.10), and have the element in row $q_{1}$ and column $q_{2}$ given by

$$
\{\mathbb{Z}, \mathbb{A}, \mathbb{X}\}_{\{\mathrm{e}, \mathrm{m}\}}^{\{x, z\}}\left(q_{1}, q_{2}\right)=\{\tilde{\zeta}, \tilde{\alpha}, \tilde{\xi}\}_{\{\mathrm{ee}, \mathrm{mm}\}}^{\{x x, y y, z z\}}\left[m\left(q_{1}\right)-m\left(q_{2}\right), n\left(q_{1}\right)-n\left(q_{2}\right)\right]
$$

Continuing the example from Table 4.1, each of the matrices from (4.17) are $9 \times 9$ matrices, e.g.

$$
\mathbb{Z}_{\mathrm{e}}^{y}=\left[\begin{array}{cccc}
\tilde{\zeta}_{\mathrm{ee}}^{y y}(0,0) & \tilde{\zeta}_{\mathrm{ee}}^{y y}\left(\begin{array}{c}
m(0)-m(1), \\
n(0)-n(1)
\end{array}\right) & \ldots & \tilde{\zeta}\left(\begin{array}{l}
m(0)-m(8), \\
n(0)-n(8)
\end{array}\right) \\
\tilde{\zeta}_{\mathrm{ee}}^{y y}\left(\begin{array}{l}
m(1)-m(0), \\
n(1)-n(0)
\end{array}\right) & \tilde{\zeta}_{\mathrm{ee}}^{y y}(0,0) & & \tilde{\zeta}\left(\begin{array}{l}
m(1)-m(8), \\
n(1)-n(8)
\end{array}\right) \\
\vdots & \ddots & \vdots \\
\tilde{\zeta}_{\mathrm{ee}}^{y y}\left(\begin{array}{l}
m(8)-m(0), \\
n(8)-n(0)
\end{array}\right) & \tilde{\zeta}_{\mathrm{ee}}^{y y}\left(\begin{array}{l}
m(8)-m(1), \\
n(8)-n(1)
\end{array}\right) & \ldots & \tilde{\zeta}_{\mathrm{ee}}^{y y}(0,0)
\end{array}\right]
$$

In the absence of modulation, these matrices are diagonal, and so the space-time harmonics corresponding to different rows/columns in (4.17a-c) are uncoupled. When space or time modulation is introduced, they couple to each other. Either way, these equations account for temporal dispersion inherent to a non-modulated surface.

Next, the expansions of the fields and polarization densities are substituted into the GSTC equations (4.5):

$$
\begin{gathered}
j \mu_{0} \Omega \tilde{\mathbf{M}}_{x}=\tilde{\mathbf{E}}_{\mathrm{t}}-\tilde{\mathbf{E}}_{\mathrm{r}}-\tilde{\mathbf{E}}_{\mathrm{i}} \\
j \epsilon_{0} \Omega \tilde{\mathbf{Q}}_{y}-j \mathbb{K}_{x} \tilde{\mathbf{M}}_{z}=\frac{1}{\eta_{0}}\left(\mathbb{K}_{z} \oslash \mathbb{K}\right)\left(-\tilde{\mathbf{E}}_{\mathrm{r}}-\tilde{\mathbf{E}}_{\mathrm{t}}+\tilde{\mathbf{E}}_{\mathrm{i}}\right)
\end{gathered}
$$

Together, (4.17a-e) provide five sets of equations to solve for the unknown fields and polarization densities, given $\tilde{\mathbf{E}}_{\mathrm{i}}$. However, each of these sets contains infinite equations, which can be truncated to $m \in[-M, M]$ and $n \in[-N, N]$ to make the problem numerically tractable. This leaves $(2 M+1)$ spatial harmonics to solve for, each of which has $(2 N+1)$ temporal harmonics. The truncation assumes that the selected number of harmonics is sufficient, and this assumption must be verified

\footnotetext{
${ }^{7} a^{\odot b}$ is the Hadamard (element-wise) power, while $a \oslash b$ is Hadamard division.
} 
after computation in the form of a series convergence. Nevertheless, each of the $(2 M+1) \times(2 N+1)$ field and polarization harmonics can be determined.

\subsection{2 "Dispersionless" Formulation}

If the temporal dispersion of the static susceptibilities is ignored, using (4.7) in the constitutive relations provides three alternative sets of equations, which replace $(4.17 \mathrm{a}-\mathrm{c})$. These are

$$
\begin{aligned}
\tilde{\mathbf{Q}}_{y} & =\frac{1}{2} \mathbb{C}_{\mathrm{e}}^{y}\left(\tilde{\mathbf{E}}_{\mathrm{r}}+\tilde{\mathbf{E}}_{\mathrm{t}}+\tilde{\mathbf{E}}_{\mathrm{i}}\right) \\
\tilde{\mathbf{M}}_{x} & =\frac{1}{2 \eta_{0}} \mathbb{C}_{\mathrm{m}}^{x}\left(\mathbb{K}_{z} \oslash \mathbb{K}\right)\left(\tilde{\mathbf{E}}_{\mathrm{r}}-\tilde{\mathbf{E}}_{\mathrm{t}}-\tilde{\mathbf{E}}_{\mathrm{i}}\right) \\
\tilde{\mathbf{M}}_{z} & =\frac{1}{2 \eta_{0}} \mathbb{C}_{\mathrm{m}}^{z}\left(\mathbb{K}_{x} \oslash \mathbb{K}\right)\left(\tilde{\mathbf{E}}_{\mathrm{r}}+\tilde{\mathbf{E}}_{\mathrm{t}}+\tilde{\mathbf{E}}_{\mathrm{i}}\right)
\end{aligned}
$$

with

$$
\mathbb{C}_{\{\mathrm{e}, \mathrm{m}\}}^{\{x, z\}}\left(q_{1}, q_{2}\right)=\tilde{\chi}_{\{\mathrm{ee}, \mathrm{mm}\}}^{\{x x, y, z z\}}\left[m\left(q_{1}\right)-m\left(q_{2}\right), n\left(q_{1}\right)-n\left(q_{2}\right)\right] .
$$

\subsubsection{Extension to an Arbitrary Incident Field}

The method presented in Sections 4.4.1 and 4.4.2 allow solving the scattering due to a plane wave excitation at an angle $\theta_{0}$, but can also be extended to arbitrary excitations, such as a single Gaussian beam (spatial distribution) or a Gaussian pulse (temporal shape). Let us denote the arbitrary incident field as $E_{\mathrm{i} \text {,tot }}^{y}(x, z, t)$. The MS responds to the field at $z=0$, where I can decompose $E_{\mathrm{i}, \text { tot }}^{y}$ into plane waves, using a Fourier transform,

$$
E_{\mathrm{i}, p q}^{y}=\frac{1}{(2 \pi)^{2}} \int_{-\infty}^{\infty} \int_{-\infty}^{\infty} E_{\mathrm{i}, \mathrm{tot}}^{y}(x, 0, t) e^{-j\left(p \omega_{\mathrm{s}} t-q k_{x s} x\right)} d x d \omega
$$

which is densely sampled (small $\omega_{\mathrm{s}}$ and $k_{x s}$ ) to yield a good approximation of the finite signal. Following this, (4.17) (with possible modification from (4.18)) can be solved for each of the plane waves with $\omega_{0}=p \omega_{\mathrm{s}}, \theta_{0}=\sin ^{-1}\left(q k_{x s} c_{0} / \omega_{0}\right)$, and $E_{0}=E_{\mathrm{i}, p q^{\prime}}^{y}$ yielding $\mathbf{E}_{a, p q}(x, z, t)$. These are simply summed to produce the scattered fields:

$$
\mathbf{E}_{\mathrm{a}, \mathrm{tot}}(x, z, t) \approx \sum_{p, q} \mathbf{E}_{\mathrm{a}, p q}(x, z, t)
$$

where $\mathrm{a}=(\mathrm{t}, \mathrm{r})$. Of course, the 2D Fourier transform (4.20) can be simplified to a 1D transform if the input signal is monochromatic or spatially uniform. 


\subsection{Results}

To demonstrate the proposed method, I consider three cases of periodically modulated surfaces: space-only modulation $\left(\omega_{\mathrm{p}}=0\right)$, time-only modulation $\left(\beta_{\mathrm{p}}=0\right)$, and general space-time modulation $\left(\omega_{\mathrm{p}} \neq 0, \beta_{\mathrm{p}} \neq 0\right)$.

\subsubsection{Space-Only Modulation}

First, I consider a spatial modulation of the electric and magnetic resonant frequencies, $\zeta_{\mathrm{ee}}^{y y}$ and $\zeta_{\mathrm{mm}}^{x x}$, of a surface with only tangential susceptibilities (from [50]), using a sawtooth profile (Fig. 4.3ab). The system of equations (4.17) is solved for a normally-incident plane wave with $M=100$ and $N=0$, for a total of 201 harmonics, producing the magnitude plotted in Fig. $4.3 \mathrm{c} .{ }^{8}$ Since the scattered fields are monochromatic, a frequency domain simulator can be used to verify the Floquet result; a finite-difference frequency-domain (FDFD) simulation was run, producing a field magnitude in agreement with the Floquet solution (Fig. 4.3d).

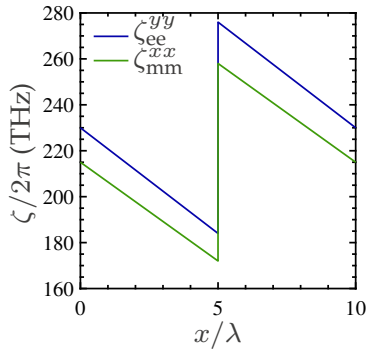

(a) Modulation

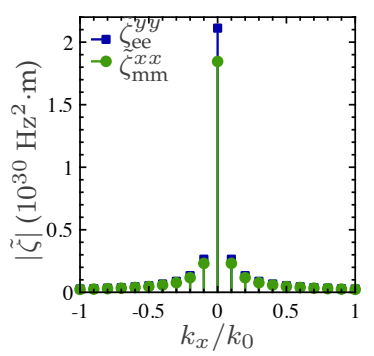

(b) Mod. harmonics

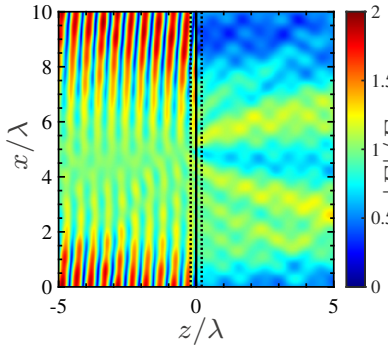

(c) $|E|$ (Floquet)

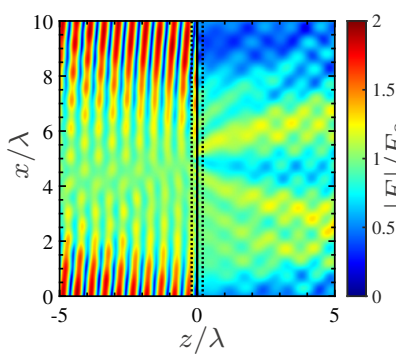

(d) $|E|($ FDFD)

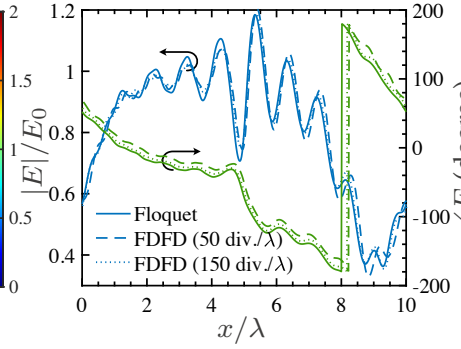

(e) Total field, $z=+\lambda / 5$

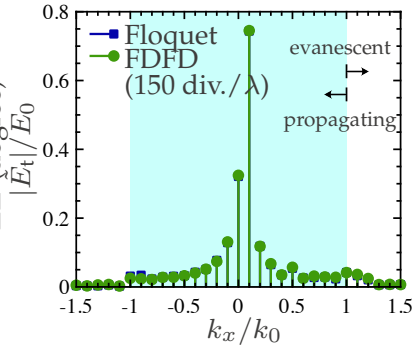

(g) Transmitted harmonics

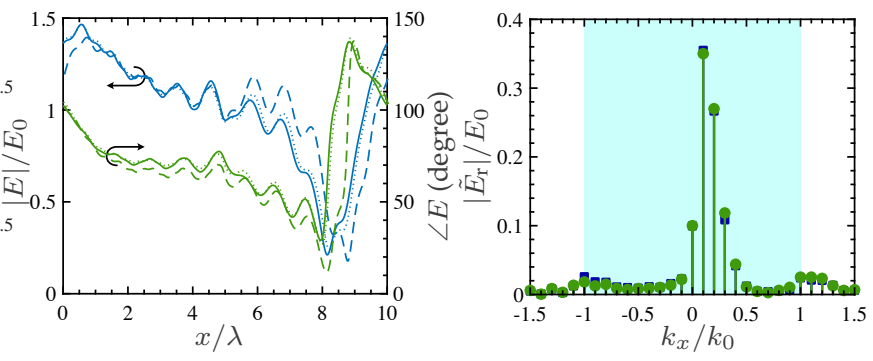

(f) Total field, $z=-\lambda / 5$

Figure 4.3 A plane wave with $\omega_{0}=2 \pi(230 \mathrm{THz})$ is normally incident on a MS where the Lorentzian resonant frequencies are spatially modulated as $\zeta_{\{\mathrm{ee}, \mathrm{mm}\}}^{\{y y, x x\}}(x)=\zeta_{\{\mathrm{ee}, \mathrm{mm}\}}^{\{y y, x x}\left[1+\Delta_{\mathrm{e}, \mathrm{m} \text { sawtooth }}\left(\beta_{\mathrm{p}} x\right)\right]$, with the modulation plotted in (a) and the $\tilde{\zeta}$ harmonics in (b). ${ }^{10}$ The total field $|E|$ over one period $0<x<L$ is shown in (c) and (d). The fields are observed along planes at $z= \pm \lambda / 5$ (dashed lines) in (e) and (f). Taking a Fourier transform produces the scattered harmonic spectra plotted in $(\mathrm{g})$ and $(\mathrm{h})$, where the incident harmonic has been subtracted from the total field. The parameters of the modulation are $\Delta_{\mathrm{e}, \mathrm{m}}=0.2$ and $\beta_{\mathrm{p}}=k_{0} / 10$, and the Lorentzian susceptibilities have nominal values $\zeta^{\prime y y}=$ $2 \pi(230 \mathrm{THz}), \zeta_{\mathrm{mm}}^{\prime x x}=2 \pi(215 \mathrm{THz}), \xi_{\mathrm{ee}}^{y y}=\xi_{\mathrm{mm}}^{x x}=301 \times 10^{9} \mathrm{rad} / \mathrm{s}$ and $\alpha_{\mathrm{ee}}^{y y}=\alpha_{\mathrm{mm}}^{x x}=7.54 \times 10^{12} \mathrm{~s}^{-1}$ as in [50].

${ }^{8}(4.17)$ also provides the amplitudes $Q^{y}(m, n), M^{x}(m, n)$, and $M^{z}(m, n)$, but usually it is the fields that are of interest and which are presented here. 
For a closer examination, the fields at observation planes at $z= \pm \lambda / 5$ are plotted in Fig. 4.3e-f. Starting with 50 divisions per wavelength, the FDFD results are seen to converge to the Floquet result as the grid is made more dense $\left(150 \mathrm{div} . / \lambda_{0}\right)$. The denser grid took 14 minutes to solve for FDFD, while the Floquet method took $0.9 \mathrm{~s}$ to compute the harmonic amplitudes, and then $7.9 \mathrm{~s}$ to calculate the fields on the same grid as for FDFD. ${ }^{11}$ Of course, the spacing of the grid does not affect the accuracy of the Floquet method which only depends on the number of harmonics. Next, a Fourier transform at the observation planes provides the harmonic amplitudes (Fig. 4.3g-h) with good agreement for both propagating harmonics $\left(\left|k_{x}\right|<k_{0}\right)$ and evanescent harmonics $\left(\left|k_{x}\right|>k_{0}\right)$, albeit the latter being small in this case. Note that even though $\theta_{0}=0^{\circ}$, the scattering primarily occurs towards $+x$ with $k_{x} \geq 0$ harmonics being dominant, which can be expected for this surface which imparts an asymmetric phase variation [64].

Next, Fig. 4.4 shows an example of a Gaussian beam incident on a surface with a cosine modulation profile. In this case, the angle of incidence is $\theta_{0}=10^{\circ}$, and the surface is designed so that the $m=1$ harmonic is scattered normally $\left(\beta_{\mathrm{p}}=k_{0} / 5.76\right.$ from (4.13)). I use a beam waist of $5 \lambda$ and decompose the field using (4.20) into 17 plane waves (found to be sufficient in representing the spatial Gaussian profile). After computing the fields for each of these plane waves and summing the total fields, the Floquet method shows good agreement with the FDFD result. The slight discrepancy between the two methods becomes smaller as the number of harmonics is increased for the Floquet method, and the FDFD mesh is made more dense.

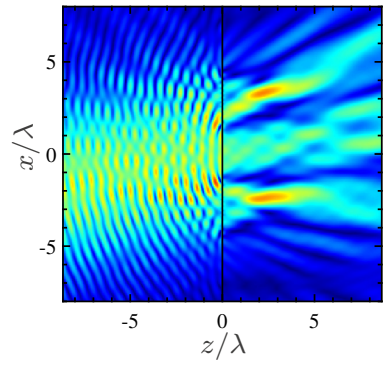

(a) $|E|$ (Floquet)

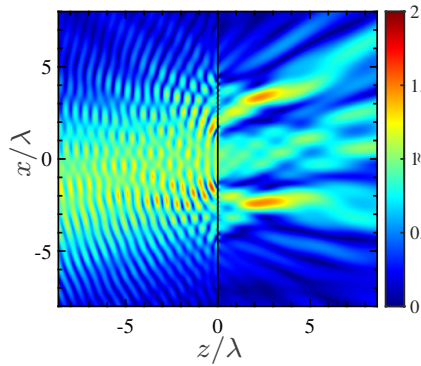

(b) $|E|($ FDFD)

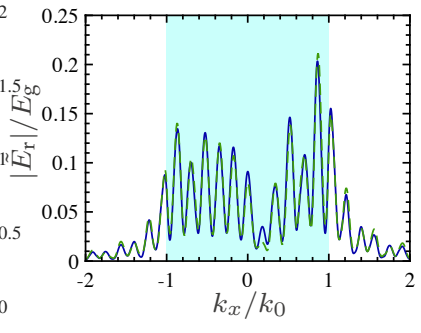

(c) Harmonic spectrum, $z=(d)$ $-\lambda / 5$

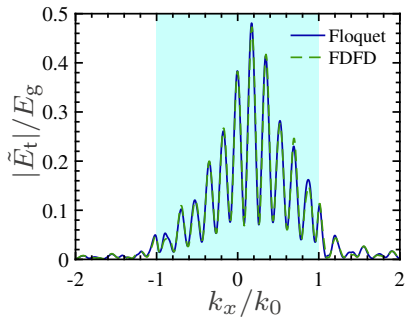

(d) Harmonic spectrum, $z=$ $+\lambda / 5$

Figure 4.4 A Gaussian beam with waist $5 \lambda$ and amplitude $E_{\mathrm{g}}$ is incident at $\theta_{0}=10^{\circ}$ onto a modulated surface with $\zeta_{\{\mathrm{ee}, \mathrm{mm}\}}^{\{y y, x x\}}(x)=\zeta_{\{\mathrm{ee}, \mathrm{mm}\}}^{\prime\{y y, x x\}}\left[1+\Delta_{\mathrm{e}, \mathrm{m}} \cos \left(\beta_{\mathrm{p}} x\right)\right]$ with $\beta_{\mathrm{p}}=k_{0} / 5.76$ and $\Delta_{\mathrm{e}, \mathrm{m}}=0.2$. The total field magnitude is plotted in (a) using the Floquet method (with $M=100$ ) and (b) using the FDFD method (with $100 \mathrm{div}$. $/ \lambda$ ). (c) shows a comparison of the scattered harmonic spectra at observation planes at $z= \pm \lambda / 5$. The nominal parameters are the same as in Fig. 4.3.

While these two examples used somewhat arbitrarily chosen parameters for the sake of demonstrating the method, let us consider next a practical implementation of a spatially-modulated MS, consisting of split metallic loops on a polyimide film (Fig. 4.5a). This provides an opportunity to test the applicability of the method for a structure with finite thickness. The loops are loaded with metal-insulator-metal (MIM) capacitors, and exhibit a strong magnetic resonance $\left(\chi_{\mathrm{mm}}^{z z}\right)$ which can be tuned around $10 \mathrm{GHz}$ by varying the side length $l$ of the capacitors, as shown in Fig. $4.5 \mathrm{~b}$. The

\footnotetext{
${ }^{11}$ These benchmarks are performed using a laptop computer with a $2.6 \mathrm{GHz} 6$-core processor, using MATLAB.
} 


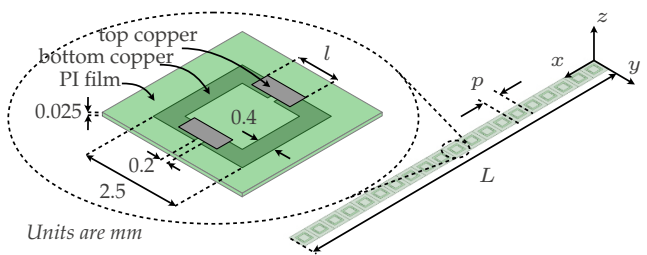

(a) Unit cell and one period of modulated MS]
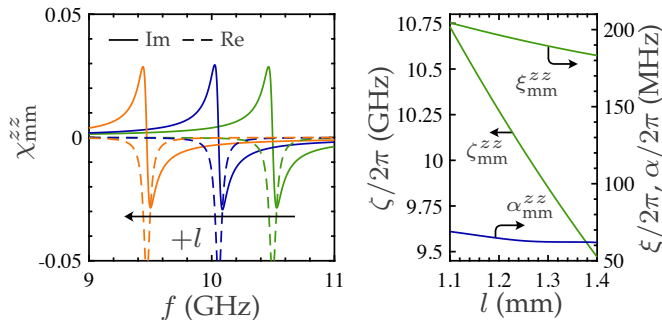

(b) Susceptibility extraction

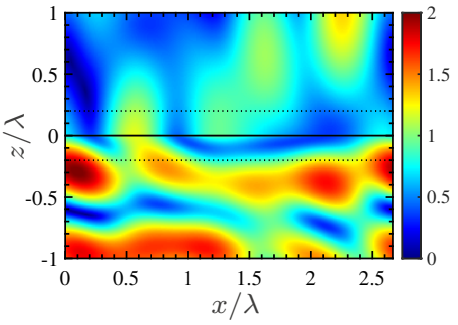

(c) $|E|$ (Floquet)

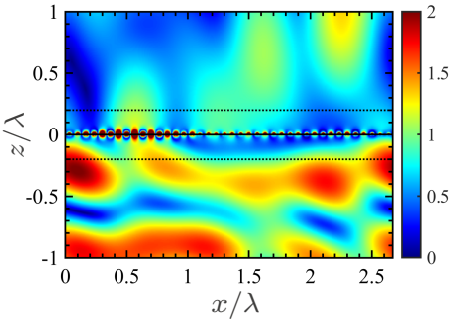

(d) $|E|$ (HFSS)

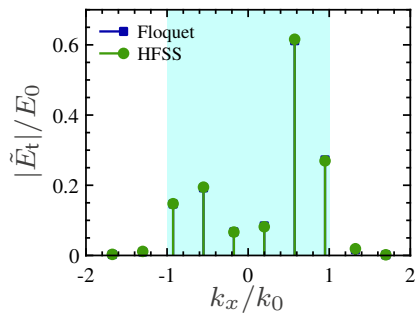

(e) Trans. harmonics $(z=+\lambda / 5)$

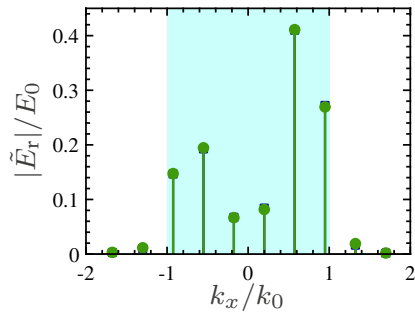

(f) Refl. harmonics $(z=-\lambda / 5)$

Figure 4.5 A surface composed of capacitively-loaded loops on a polyimide (PI) film $\left(\epsilon_{r}=3.4\right.$, loss tangent 0.002$)$ was modelled in HFSS, where in each cell the side length of a MIM capacitor $l$ was modulated. The modulated surface in (a) was composed of 20 cells having a side length $p=4 \mathrm{~mm}$ and a total modulation period $L=20 p$, using periodic boundaries to emulate an infinite surface. The susceptibility of an individual cell was extracted for different $l$ with the dominant $\chi_{\mathrm{mm}}^{z z}$ plotted in (b) along with the corresponding Lorentzian parameters. Meanwhile, $\chi_{\mathrm{mm}}^{x x}=0$ and $\chi_{\mathrm{ee}}^{y y}$ is constant: $\zeta_{\mathrm{ee}}^{y y}=2 \pi(54.5 \mathrm{GHz}), \xi_{\mathrm{ee}}^{y y}=2 \pi(1.90 \mathrm{GHz})$, and $\alpha_{\mathrm{ee}}^{y y}=2.71 \times 10^{9} \mathrm{~s}^{-1}$. Having the extracted parameters, a modulation of $l(x)=\left[1.25 \mathrm{~mm}+(0.05 \mathrm{~mm}) \cos \left(\beta_{\mathrm{p}} x\right)\right]$ was applied and a $10 \mathrm{GHz}$ incident plane wave was launched at $\theta_{0}=35^{\circ}$, with the total field magnitude with the Floquet method ( $\left.M=100\right)$ plotted in (c) and the full-wave HFSS fields in (d). (e) and (f) show a comparison of the scattered harmonics calculated at observation planes at $z= \pm \lambda / 5$.

susceptibilities were extracted for different lengths $l$ using full-wave HFSS simulations of the unit cells with periodic boundary conditions and using plane-wave incidence at two different angles as prescribed in [12], also revealing a small $\chi_{\mathrm{ee}}^{y y}$ in addition to $\chi_{\mathrm{mm}}^{z z}$. By curve-fitting, the Lorentzian parameters were extracted as a function of $l$, which provided a look-up table when this parameter was modulated with a period consisting of 20 cells. Varying the parameter $l$ results in a change of all three parameters (resonant frequency, plasma frequency, and loss), taken into account in the proposed method. A full-wave HFSS simulation of the complete modulated surface was performed, taking 6 hours, while the Floquet computation by itself required $1.3 \mathrm{~s}$ using $M=100$, on the same desktop computer. For a fair comparison, note that the extraction procedure takes about 1.5 hours for three different $l$ values, which in this case is found to be sufficient to capture the smooth variation of the Lorentzian parameters with $l$. Thus, the Floquet method is still faster including this one-time computation cost of extraction. More importantly, the primary benefit of the Floquet method would be that once the extraction is complete, any arbitrary modulation can be rapidly computed on-demand, while a time-intensive full-wave simulation of new physical structure is needed otherwise.

The HFSS fields (Fig. 4.5c) show a rapid variation close to the surface; this is due to the HFSS model 
capturing the unit cell in detail, and therefore the fields varying strongly within the unit cell. The surface characterization using susceptibilities does not capture this as it is a homogenization that predicts the average fields over the scale length of a unit-cell, also called the "macroscopic" fields in the literature [10]. It does this accurately, with nearly perfect agreement when considering the harmonic spectra in Fig. 4.5e-f. This example thus clearly demonstrates that the zero-thickness sheet model with space-varying Lorentzian parameters goes beyond just a theoretical analogue of a practical MS, and in fact represents an efficient and an accurate field analysis tool.

\subsubsection{Time-Only Modulation}

Now, I turn to the temporal modulation of the MS. Since the surface is uniform and the incident field is a normal plane wave, this reduces to a 1D problem. To validate the Floquet solution, I used a FDTD technique where the susceptibility is time-variant [49], and ran the simulation until a steadystate was achieved. Then, a Fourier transform provided the time harmonics that were generated. Fig. 4.6 shows the time-domain waveforms obtained using the Floquet and FDTD methods, for an example of a weak modulation (cosine profile with $\Delta_{\mathrm{e}, \mathrm{m}}=0.2$ ), where the waveforms are recorded once the steady-state is reached in FDTD. In this case, the incident field at $\omega_{0}=2 \pi(130 \mathrm{THz})$ is far away from the resonances (i.e. $215 \mathrm{THz}$ and $230 \mathrm{THz}$ ) so it might be assumed that the static temporal dispersion may possibly be neglected. The waveform using the "dispersionless" method (4.18) is plotted, showing slight agreement but nevertheless deviating significantly from the Lorentzian Floquet (4.17a-c) and the baseline FDTD results (Fig. 4.7a), which themselves are nearly identical.

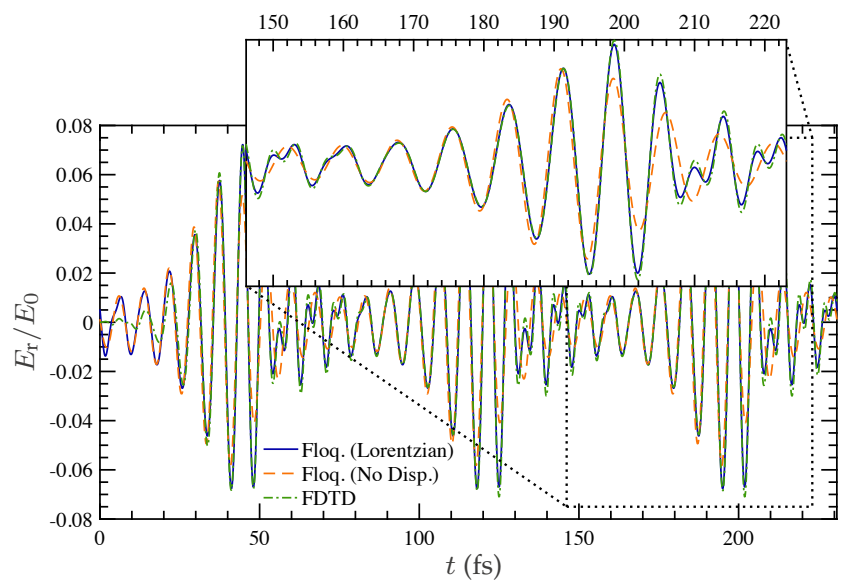

Figure 4.6 Shows the reflected field at the surface $\left(z=0^{-}\right)$for a uniform surface that is time-modulated with an incident plane wave at $\omega_{0}=2 \pi(130 \mathrm{THz})$ that is far-away from the resonant frequencies of the surface susceptibilities. The results for Floquet method incorporating Lorentzian dispersion (4.17a-c) and the "disperionless" method (4.18) are plotted, both using $N=100$. For validation, an FDTD simulation is run until it reaches a steady state (inset), ending after $250 \mathrm{fs}$, and using 1000 divisions/period. The transmitted field is treated likewise. The resonant frequencies are modulated as $\zeta_{\{\mathrm{ee}, \mathrm{mm}\}}^{\{y y, x x\}}(t)=\zeta_{\{\mathrm{ee}, \mathrm{mm}\}}^{\{y y, x x\}}\left[1+\Delta_{\mathrm{e}, \mathrm{m}} \cos \left(\omega_{\mathrm{p}} t\right)\right]$, with $\omega_{\mathrm{p}}=\omega_{0} / 10$ and $\Delta_{\mathrm{e}, \mathrm{m}}=0.2$.

Operating so far away from resonance produces insignificant scattering in this case, but this result is included to demonstrate that the "dispersionless" method works only if the susceptibility has a 
weak frequency-dependence, generally requiring operation away from resonances. This naturally means weaker interaction of the incident fields with the MSs resulting in small scattered fields. Next, the incident frequency is shifted to $230 \mathrm{THz}$, in which case the "dispersionless" method completely fails and is thus not plotted. However, the Lorentzian Floquet method shows agreement with an FDTD simulation. The latter took $3.1 \mathrm{~s}$ while the Floquet method took $1.3 \mathrm{~s}$ to calculate the harmonic amplitudes followed by $0.5 \mathrm{~s}$ to calculate the fields, and so for a simple $1 \mathrm{D}$ case, both methods were quick and neither was clearly faster.

Finally, I consider a stronger modulation $\left(\Delta_{\mathrm{e}, \mathrm{m}}=0.5\right)$ while also increasing the pumping frequency to $\omega_{\mathrm{p}}=\omega_{0} / 2$. In this case, harmonics at negative frequencies are excited in the Floquet solution ("raw harmonics" in Fig. 4.7c). By taking a Fourier transform of the time-domain Floquet waveform (orange diamonds), these can be "flipped" to positive frequencies; in this case, they combine with positive frequency harmonics because $\omega_{\mathrm{p}}$ is an integer multiple of $\omega_{0}$. However, even with this taken into account, there is a discrepancy that is observed with the FDTD result.

To determine which result is more accurate, consider the equations they should satisfy, i.e. (4.5) and (4.9). I can numerically compute the derivatives $d Q^{y} / d t$ and $d^{2} Q^{y} / d t^{2}$ using the time domain waveforms of FDTD and Floquet methods and substitute into (4.9a) to find a new expression $E_{\mathrm{av}}^{y}$ '. Similarly, (4.5a) yields a new expression $\Delta E^{y^{\prime}}$. Finally, I solve

$$
\begin{aligned}
& E_{\mathrm{t}}^{\prime}-\left(E_{\mathrm{r}}^{\prime}+E_{\mathrm{i}}\right)=\Delta E^{y \prime}, \\
& \frac{1}{2}\left(E_{\mathrm{t}}^{\prime}+E_{\mathrm{r}}^{\prime}+E_{\mathrm{i}}\right)=E_{\mathrm{av}}^{y \prime},
\end{aligned}
$$

to find new values $E_{\mathrm{r}}^{\prime}$ and $E_{\mathrm{t}}^{\prime}$. If the solution is exact, then I should have $E_{\mathrm{r}}^{\prime}=E_{\mathrm{r}}$ and $E_{\mathrm{t}}^{\prime}=E_{\mathrm{t}}$. I carry out this procedure for both the Floquet and FDTD methods, with the disprency $\left|E_{\mathrm{r}}-E_{\mathrm{r}}^{\prime}\right|$ shown in Fig. 4.8. While the FDTD discrepancy changes slowly as time-stepping becomes more fine, the Floquet solution shows convergence as the number of harmonics increases. The lack of improvement in the FDTD result may be due to the computational challenges of the system, which is essentially two coupled resonators, and there may be alternative FDTD implementations which work better. Compared to this FDTD implementation, the Floquet solution has a much smaller discrepancy, indicating that it is the more accurate solution among the two, of the original field equations.

Also, see that for both methods, the DC electric field harmonics at $\omega(n)=0$ are zero (the magnetic field, not shown, is likewise zero). From a physical perspective, a DC $H$ field (or $E$ field) difference can be generated across a boundary due to a static electric current (magnetic current), with the well-known boundary condition derived from Ampere's law (Faraday's law) [54, p. 76]. In the right hand side of (4.5), this corresponds to polarizations that are linearly changing over time, which is in contradiction with a periodic solution. Mathematically, this manifests itself in (4.17d), which requires $\tilde{E}_{\mathrm{t}}(m, n)-\tilde{E}_{\mathrm{r}}(m, n)=0$ for $\omega(n)=0$, while $(4.17 \mathrm{e})$ requires $\tilde{E}_{\mathrm{t}}(m, n)+\tilde{E}_{\mathrm{r}}(m, n)=0$. The solution, of course, is that the DC fields are zero. 

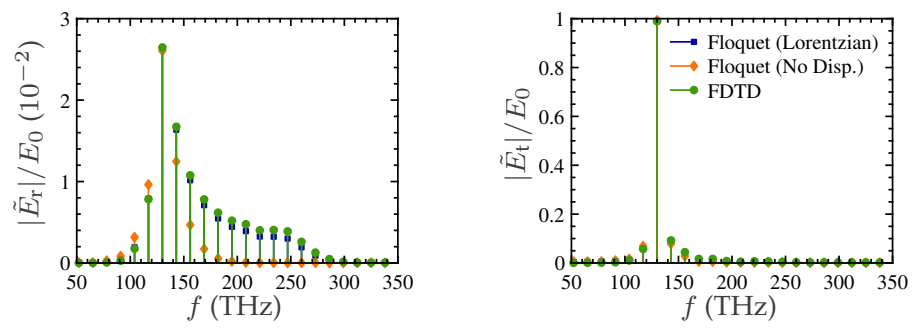

(a) $\omega_{0}=2 \pi(130 \mathrm{THz}), \omega_{\mathrm{p}}=\omega_{0} / 10, \Delta_{\mathrm{e}, \mathrm{m}}=0.2$
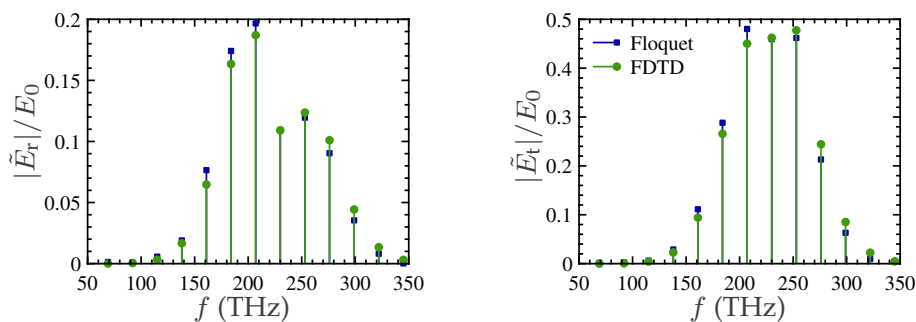

(b) $\omega_{0}=2 \pi(230 \mathrm{THz}), \omega_{\mathrm{p}}=\omega_{0} / 10, \Delta_{\mathrm{e}, \mathrm{m}}=0.2$
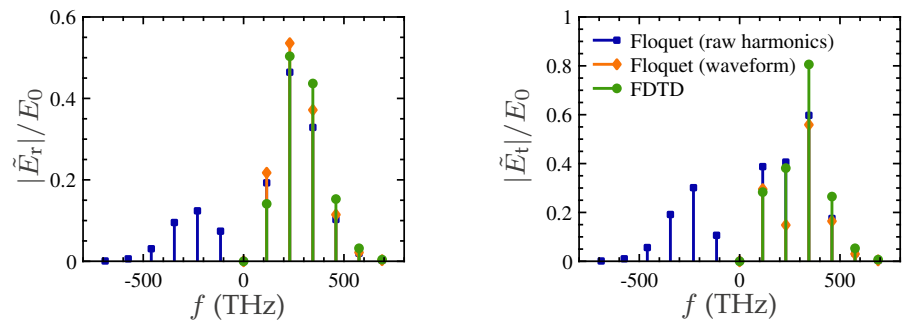

(c) $\omega_{0}=2 \pi(230 \mathrm{THz}), \omega_{\mathrm{p}}=\omega_{0} / 2, \Delta_{\mathrm{e}, \mathrm{m}}=0.5$

Figure 4.7 Shows three cases of uniform MSs that are time-modulated with $\zeta_{\{\mathrm{ee}, \mathrm{mm}\}}^{\{y y x\}}(t)=\zeta_{\{\mathrm{ee}, \mathrm{mm}\}}^{\{y y, x x\}}\left[1+\Delta_{\mathrm{e}, \mathrm{m}} \cos \left(\omega_{\mathrm{p}} t\right)\right]$. In each of the three cases (a-c), the incident field frequency and modulation parameters are varied. In (b), the Floquet solution produces negative frequency harmonics, which can be "folded" into the positive frequency components, producing the half-sided spectrum in orange diamonds. The form of the modulation is the same as in Fig 4.6. 


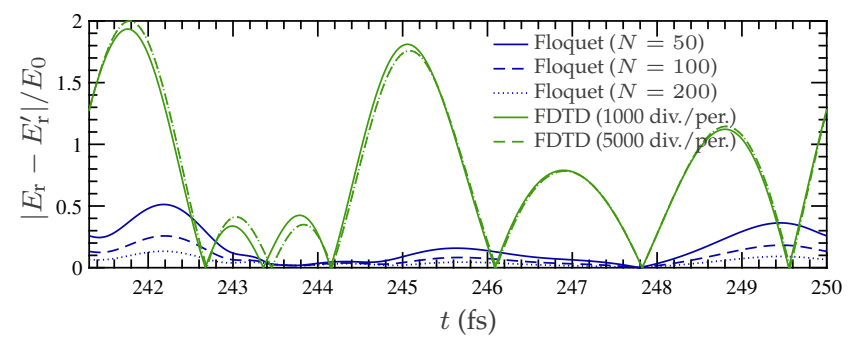

Figure 4.8 To examine the discrepancy between the FDTD and Floquet results in Fig. 4.7c, the consistency of the solutions was considered. The FDTD discrepancy is larger than the discrepancy of the Floquet result, while the latter improves as the number of harmonics $(2 N+1)$ is increased.

\subsubsection{Space-Time Modulation}

Finally, consider the general case of space-time modulation. Here, it is convenient to use generalized S-parameters [65] to describe the system, where each harmonic in the transmission and reflection regions can be considered a port, for a total of $2(2 N+1)(2 M+1)$ when the fundamental harmonic $(m, n)=(0,0)$ is normally incident. ${ }^{12}$ I will label the reflection parameter

$$
R_{m_{1} n_{1}}^{m_{2} n_{2}}=\left.\frac{\tilde{E}_{\mathrm{r}}\left(m_{2}, n_{2}\right)}{\tilde{E}_{\mathrm{i}}\left(m_{\mathrm{i}}, n_{\mathrm{i}}\right)}\right|_{\left(m_{\mathrm{i}}, n_{\mathrm{i}}\right)=\left(-m_{1}, n_{1}\right)}
$$

which is measured by evaluating (4.17) with the harmonic $\left(m_{\mathrm{i}}, n_{\mathrm{i}}\right)=\left(-m_{1}, n_{1}\right)$ excited ${ }^{13}$ with a plane wave and the $\left(m_{2}, n_{2}\right)$ port probed. That is, this represents the scattering from port $\left(m_{1}, n_{1}\right)$ to $\left(m_{2}, n_{2}\right)$, with $m$ as a spatial index and $n$ as a frequency index that can be used in (4.13) and (4.12c) to find the direction and frequency, respectively. Similarly, the transmission parameter is

$$
T_{m_{1} n_{1}}^{m_{2} n_{2}}=\left.\frac{\tilde{E}_{\mathrm{t}}\left(m_{2}, n_{2}\right)}{\tilde{E}_{\mathrm{i}}\left(m_{\mathrm{i}}, n_{\mathrm{i}}\right)}\right|_{\left(m_{\mathrm{i}}, n_{\mathrm{i}}\right)=\left(-m_{1}, n_{1}\right)}
$$

With this convention, Fig. 4.9 shows a case where the space and time dependencies are decoupled, and the modulation resembles a standing wave. Each pixel represents an S-parameter with port $\left(m_{1}, n_{1}\right)=(1,0)$ excited in (a) and $(-1,0)$ excited in (b). One primary interest is whether or not this represents a reciprocal system. One way to approach this is to evaluate if $R_{m_{1} n_{1}}^{m_{2} n_{2}}=R_{m_{2} n_{2}}^{m_{1} n_{1}}$ and $T_{m_{1} n_{1}}^{m_{2} n_{2}}=T_{m_{2} n_{2}}^{m_{1} n_{1}}$ for all ports combinations [65]. For example, see that $\left|T_{1,0}^{-1,0}\right|=\left|T_{-1,0}^{1,0}\right|=0.20$ from Fig. 4.9, so these ports are reciprocal.

Alternatively, I can consider the Onsager-Casimir relations, which place conditions on the constitutive relations of LTV systems for reciprocity $[65,66]$. In the case at hand, these require $\chi_{\{\text {ee,mm }\}}^{\{y y, x x\}}\left(v_{\mathrm{p}}\right)=$

\footnotetext{
${ }^{12}$ This also happens if the fundamental is at $\theta_{0}=\sin ^{-1}\left(m \beta_{\mathrm{p}}\right)$ with some integer $m$. Otherwise, there will in general be twice as many ports because of a lack of symmetry across the $z$-axis with the incident and reflected field propagation directions.

${ }^{13}$ I set $m_{\mathrm{i}}=-m_{1}$ so that the incident $\left(m_{\mathrm{i}}\right)$ and reflected $\left(m_{2}\right)$ wavevectors are parallel, but opposite in direction. Thus, they correspond to the same port in space and frequency.
} 

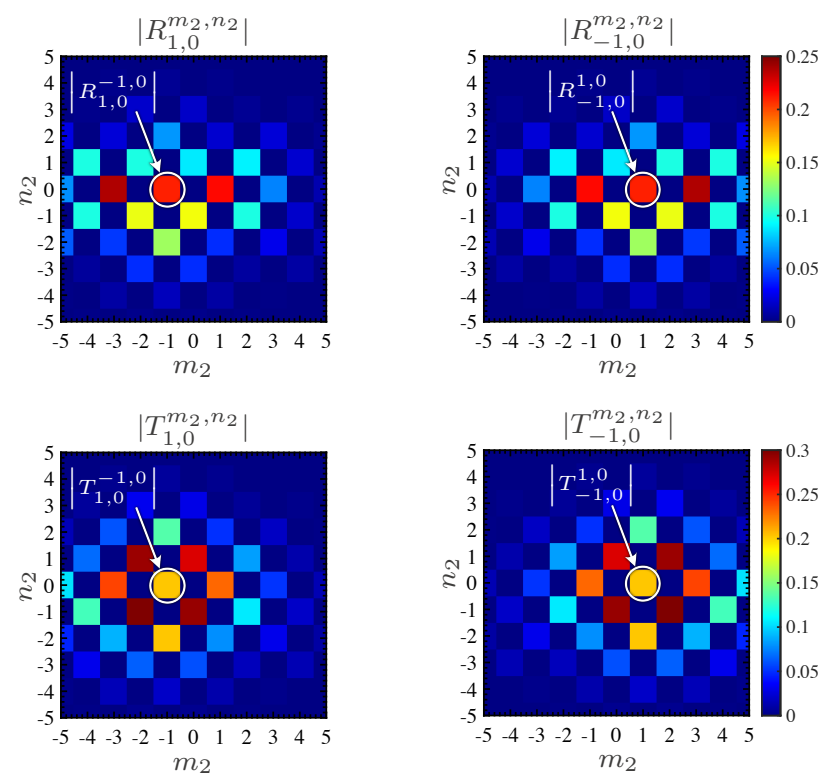

(a) $\left(m_{1}, n_{1}\right)=(1,0)$

(b) $\left(m_{1}, n_{1}\right)=(-1,0)$

Figure 4.9 Reciprocal space-time modulation where $\zeta_{\{\mathrm{ee}, \mathrm{mm}\}}^{\{y y, x x\}}(x, t)=\zeta_{\{\mathrm{ee}, \mathrm{mm}\}}^{\{y y, x x\}}\left[1+\Delta_{\mathrm{e}, \mathrm{m}} \cos \left(\omega_{\mathrm{p}} t\right) \cos \left(\beta_{\mathrm{p}} x\right)\right]$ and space and time dependencies are decoupled, with different ports $\left(m_{1}, n_{1}\right)$ excited in (a) and (b). Each pixel represents a space-time harmonic, calculated using the Floquet method. For both cases, a plane wave is incident with $f_{0}=230 \mathrm{THz}, \Delta_{\mathrm{e}, \mathrm{m}}=0.2$, $\omega_{\mathrm{p}}=\omega_{0} / 10$, and $\beta_{\mathrm{p}}=k_{0} / 5.76$ so that the first space harmonic is at $\theta(1,0)=10^{\circ}$. The nominal parameters are the same as in Fig. 4.3.

$\chi_{\{\mathrm{ee}, \mathrm{mm}\}}^{\{y y, x\}}\left(-v_{\mathrm{p}}\right)$ in order for the system to be reciprocal, where the susceptibilities are a function of $v_{\mathrm{p}}$, which is the velocity of the modulation. Hence, the modulated parameter must also be identical when the direction of modulation is reversed. This is indeed the case for the standing wave modulation, which can be written as the sum of two waves travelling in opposite directions, where switching this sign of velocity is inconsequential $\left(\chi_{\{\mathrm{ee}, \mathrm{mm}\}}^{\{y, x x\}}\left(x, t, v_{\mathrm{p}}\right)=\chi_{\{\mathrm{ee}, \mathrm{mm}\}}^{\{y y, x\}}\left(x, t,-v_{\mathrm{p}}\right)\right)$. This can be intuitively understood as follows: the surface "appears" the same to an incident wave regardless of which side of the surface it approaches from.

To break reciprocity, I consider a case where the space and time modulations are coupled in the form of a wave travelling along the surface in the $+x$ direction. The S-parameters are in Fig. 4.10, where in (a), I see that exciting the $(1,0)$ port I observe an up-converted transmitted harmonic at $(0,1)$ with $\left|T_{1,0}^{0,1}\right|=0.47$. Exciting this port in hand, I find $\left|T_{0,1}^{1,0}\right| \approx 0$ ! (Instead port $(-1,0)$ is excited.) Thus the system is non-reciprocal. Of course, the Onsager-Casimir relations are not satisfied in this case, since the direction of modulation is critical.

Extending the analysis from a plane wave to a more general incident field, and at the same time visually demonstrating the non-reciprocity, Fig. 4.10c-d show the total fields at several harmonic frequencies when a Gaussian beam is launched at the MS in the same two experiments. In the first case $(\mathrm{c})$, a beam incident on the $(1,0)$ port $\left(10^{\circ}\right.$ at $\left.1.0 \omega_{0}\right)$ has a normally transmitted harmonic that is up-converted $\left(1.1 \omega_{0}\right)$, corresponding to port $(0,1)$. If I in turn excite this port in (d), i.e. 

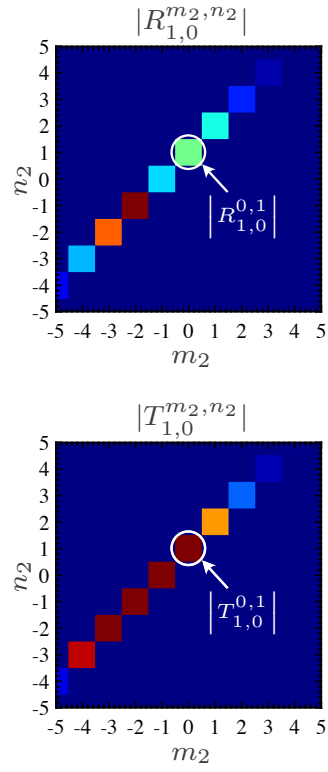

(a) $\left(m_{1}, n_{1}\right)=(1,0)$
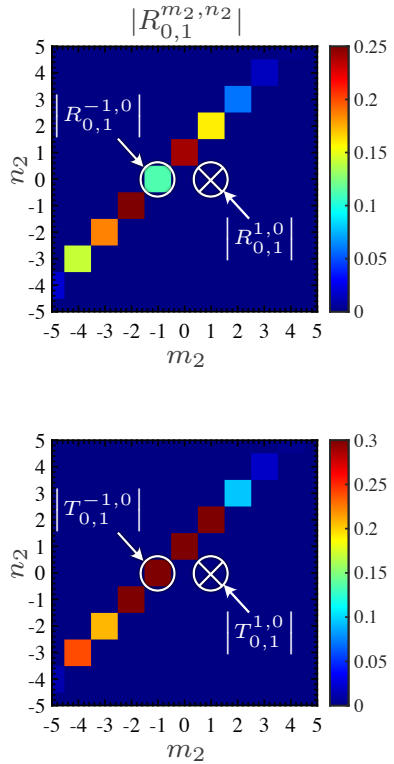

(b) $\left(m_{1}, n_{1}\right)=(0,1)$

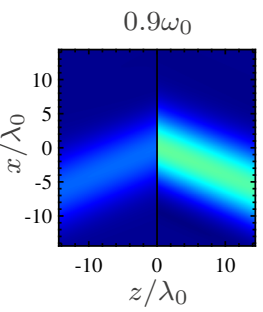

$1.0 \omega_{0}$

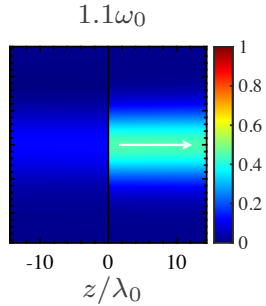

(c) Excitation from $(1,0)$ port on left side

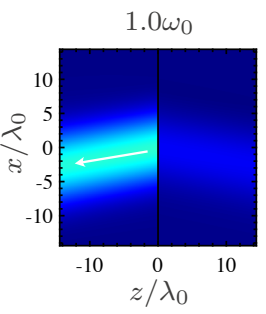

$1.1 \omega_{0}$
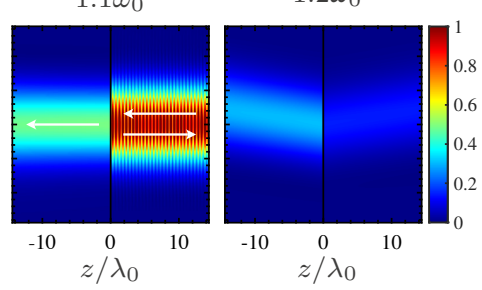

(d) Excitation from $(0,1)$ port on right side

Figure 4.10 Non-reciprocal space-time modulated MS where $\zeta_{\text {\{ee,mm\} }}^{\{y y, x x\}}(x, t)=\zeta_{\{\mathrm{ee}, \mathrm{mm}\}}^{\prime\{y, x x\}}\left[1+\Delta_{\mathrm{e}, \mathrm{m}} \cos \left(\omega_{\mathrm{p}} t-\beta_{\mathrm{p}} x\right)\right]$ and space and time dependencies are coupled. The harmonic amplitudes are plotted in (a) and (b) with different ports excited. In (c) and (d) the total fields at given frequencies, when the incident field is a Gaussian beam with a waist $5 \lambda_{0}$ $\left[\omega_{0}=2 \pi(230 \mathrm{THz})\right]$. Aside from the form of the modulation, the parameters are the same as in Fig. 4.9.

with an incident beam at $1.1 \omega_{0}$ and $0^{\circ}$, I do not find a transmitted harmonic in the direction of the first incident beam at $\omega_{0}$. This harmonic at $1.0 \omega_{0}$ is instead directed at an angle $-10^{\circ}$, clearly demonstrating the nonreciprocal nature of the surface.

\subsection{Conclusions \& Future Work}

A rigorous semi-analytical Floquet analysis has been presented for a zero-thickness space-time modulated MS using GSTCs to model and determine the strengths of the new harmonic components of the scattered fields. I have accounted for the dispersion inherent to the static MS using physically-motivated Lorentzian susceptibilities, with parameters that are modulated in space and time. To correctly account for the angular scattering of the MSs, both tangential and normal surface susceptibilities have been considered with their respective modulations. The Lorentzian parameters $(\zeta, \alpha$, and $\xi)$ can take on arbitrary periodic profiles in space and time, for both the electric and magnetic susceptibilities. The validity of the method has been established with comparison to FDFD simulations for pure-space modulation and FDTD simulations for pure-time modulation. Moreover, taking a practical MS with finite thickness as an example, the validity and the practical usefulness of the zero-thickness dispersive sheet model has been further established. In addition, it has been further demonstrated that a dispersion-less formulation in cases of temporal modulation, especially when the MS is operated near its resonance, is a gross approximation. Including 
dispersion effects of the static (non-modulated) susceptibility, as has been done in this work, is thus critical for correct calculation of the scattered fields. Finally, two cases of space time modulation were presented: a standing wave perturbation which was found to be reciprocal and a traveling wave perturbation that breaks Lorentz reciprocity.

The method was presented for TE-polarized fields propagating in the $x-z$ plane. Future work could include extending the approach to arbitrary polarization and propagation. Another direction for future work could be the realization of a unit cell which has temporal modulation at a frequency comparable to the incident field. Nevertheless, the proposed method is fast, simple, and versatile, and is expected to be a useful tool for designing general periodic and non-reciprocal MSs. 


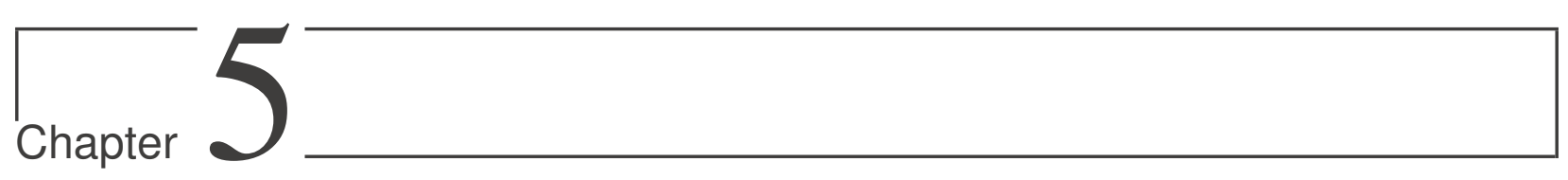

\section{EXPERIMENTAL NEAR-FIELD \\ CHARACTERIZATION OF METASURFACES}

MSs are generally designed with a combination of simulations and models such as equivalent circuits or surface susceptibilities. These allow the designer to quickly calculate the scattered electric fields, given an incident field. Following fabrication, there are multiple approaches to the characterization of the MS, depending on the quantities which are desired. If the scattered far-fields are desired, an angular scan can be performed in an anechoic chamber. On the other hand, if the complex electric field distribution next to the metsurface is desired, it can be probed using a near-field (NF) scanning system. Complementary to the theory presented in Chapters 2-4, this final chapter is a experimental demonstration of the Floquet method, using a NF system which was developed as a contribution of this thesis. While the method has been shown to be accurate through extensive comparison to alternative simulations (FDFD, FDTD, BEM, and full-wave HFSS), here I show a practical implementation of a simple metasurface, and the measurement of the near-fields.

The structure of this chapter is as follows. Section 5.1 provides an introduction to MS NF measurements, and is followed by a description of the system which was developed, in Section 5.2. Section 5.3 presents a simple modulated metasurface which was fabricated and measured, with the results compared to the Floquet method. While a single fixed transmitting horn antenna was used for the presented results in Section 5.3, Section 5.4 shows how it is possible to reconstruct arbitrary field incident fields using straight-forward but novel technique. Finally, conclusions are presented in Section 5.5.

\subsection{Introduction}

A typical NF scanning system is schematically depicted in Fig. 5.1a [67]. In the context of MSs, such a system works by illuminating the MS with an incident field $\mathrm{E}_{\mathrm{i}}(x, z)$ generated with a fixed antenna (Tx) with its aperture placed at $\left(x_{\mathrm{ap}}, z_{\mathrm{ap}}\right)$. The transmitted fields next to the MS, $\mathrm{E}_{\mathrm{t}}(x, z)$, 
are probed with a waveguide probe antenna $(\mathrm{Rx})$ which can perform a scan along a line, plane, or volume and record the field at a collection of points $\left(x_{\mathrm{rx}}, z_{\mathrm{rx}}\right)$. With this setup, it is not possible to probe the reflected fields. Absorbing material is installed to prevent unwanted reflections for an accurate characterization of the MS, and can also be installed adjacent to the surface to eliminate diffraction around the edges, as depicted in Fig. 5.1a. An example of such a system-the one developed as a contribution of this thesis for the Metamaterials and Antennas Research Squad (MARS) at Carleton University-is in Fig. 5.1b, operating from $26.5 \mathrm{GHz}$ to $40 \mathrm{GHz}$ with Eravent antenna models SAR-2013-28-S2 and SAP-28-R2 for Tx and Rx, respectively. Compared to the standard farfield characterization, the prime benefit of a near-field system is its compact size and capability of measuring the detailed complex transmittance of the surface. The near-fields nevertheless can be used to compute the far-fields using standard near-to-farfield transformation procedures, if they are desired [67].

An important caveat must be noted. The analysis presented in Chapter 4 considers invariance along the $y$-axis; i.e. a 2-D problem. Meanwhile, the measurement system is inherently 3-D, and so the 2-D analysis does not strictly apply. For rigorous modelling, the Floquet methodology would need to be extended to include spatial harmonics along $y$, and full 3-D HFSS simulations would be significantly more computationally expensive. However, if the MS which is uniform along the $y$-axis while the horn produces a 3-D incident field, a 2-D approximation can be useful if the fields are measured in the $x-z$ plane, with the illuminating horn also placed at this plane of reflection symmetry, as will be demonstrated in this chapter.

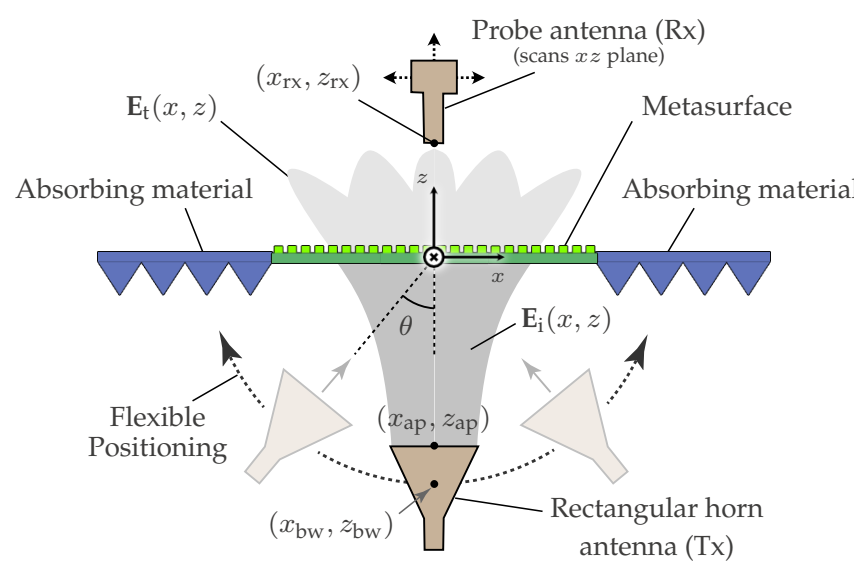

(a) Schematic

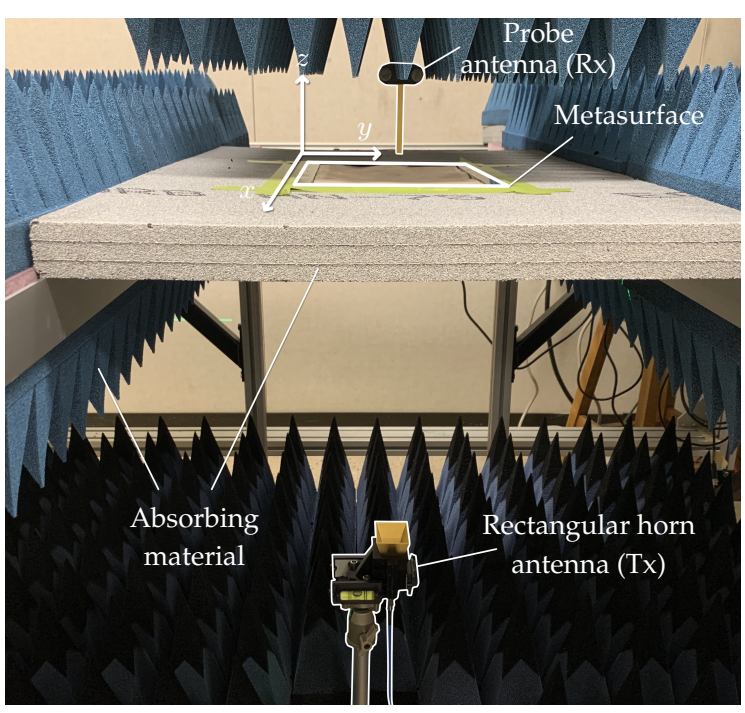

(b) Near-field system at Carleton University, configured for TE polarization.

Figure 5.1 Near-field system for transmissive metasurface characterization. 


\subsection{Development of a Custom NF System}

\subsubsection{System Overview}

The automated measurement of near fields requires two tasks to be performed:

1. Measurement of S-parameters of two ports, connected to the Tx and Rx antennas, with a vector network analyzer (VNA).

2. Movement of the Rx probe antenna along the $x, y$, and $z$ axes, while stopping at precise discrete points, where the S-parameters are recorded, with a digital motion controller (DMC).

The VNA used is a Keysight $8722 \mathrm{ES}$ ( $50 \mathrm{MHz}$ to $40 \mathrm{GHz}$ ), with a low-noise amplifier (LNA), Ervavent model SBL-2034433040-2F2F-S1, placed before port 2 to stay within the dynamic range of the VNA. Meanwhile, the DMC which was used is model DMC4163 from Galil. This is connected to four stepper motors, assigned to the three axes.

The VNA and DMC are controlled with custom Python code running on a PC, which automates the measurements and is connected to the VNA and DMC with USB (Figure 5.2). For the mechanical frame and moving stage, a re-purposed CNC machine was used, shown in Figure 5.3. It has magnetic end-stop sensors on each axis, which allow for precise calibration of the position.

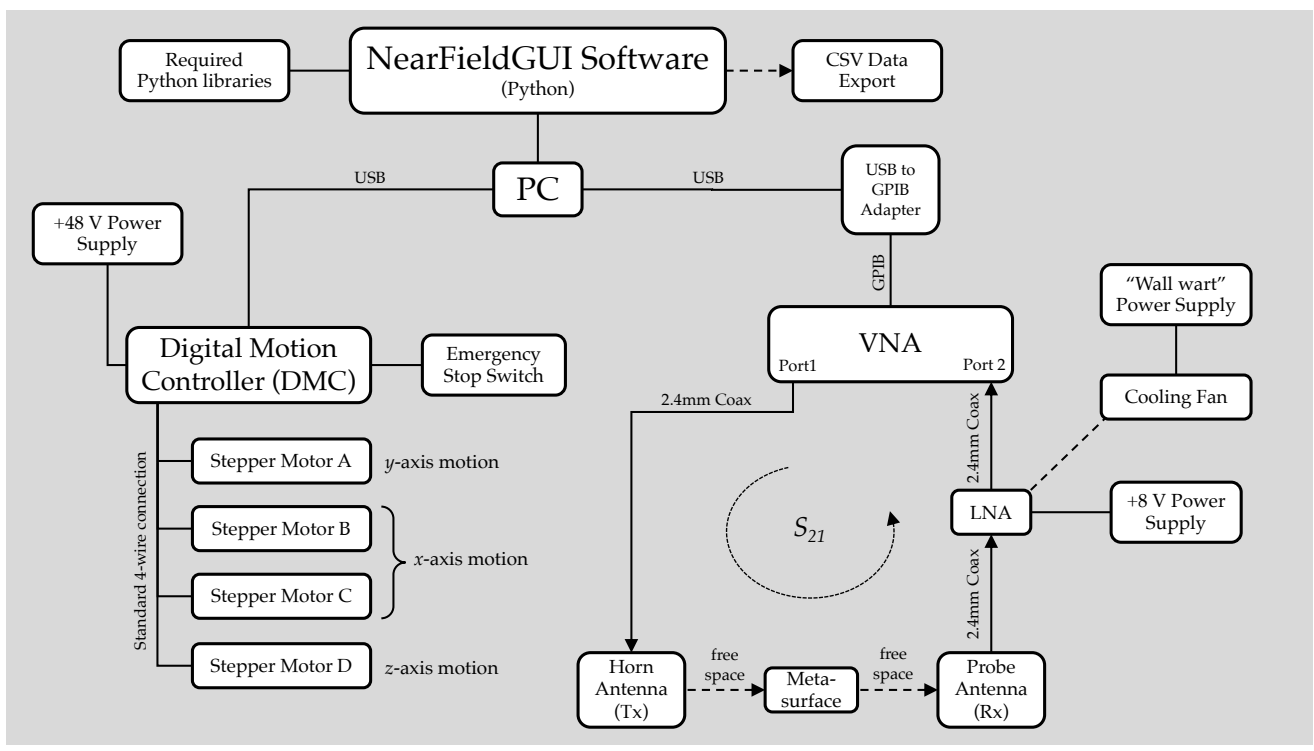

Figure 5.2 A block diagram of the developed NF system. 


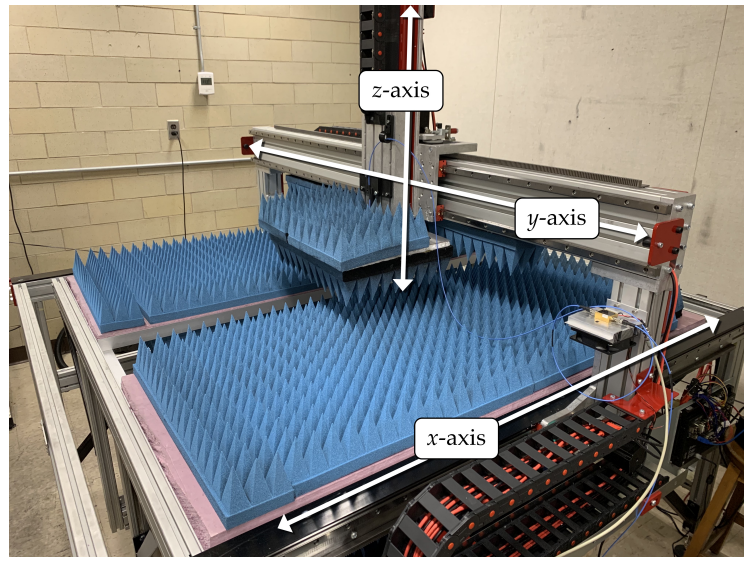

(a) CNC Frame Axes

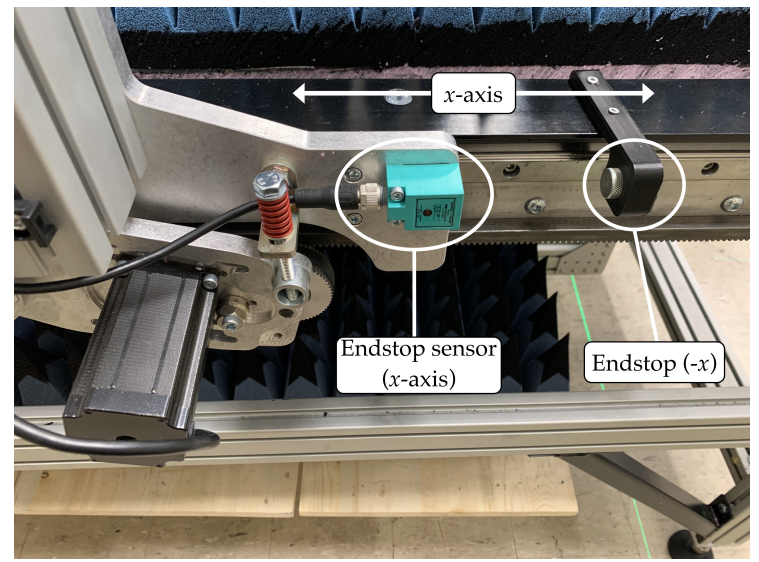

(b) $x$-axis end-stop mechanism

Figure 5.3 The CNC frame allows for scanning along three axes, controlled by stepper motors, while end-stop sensors allow for precise calibration of the position.

\subsubsection{Alignment}

When performing measurements, it is critical to align the antennas and MS as precisely as possible. To this end, a 3-plane laser was used, shown in Figure 5.4a. The alignment was performed relative to the CNC frame, placing the Tx antenna, Rx antenna, and midpoint of the metasurface, on the same $x-z$ plane. Furthermore, the Tx antenna is mounted on a tripod with a custom 3D-printed piece (5.4b), which has bubble levels to help with alignment, along with another laser.

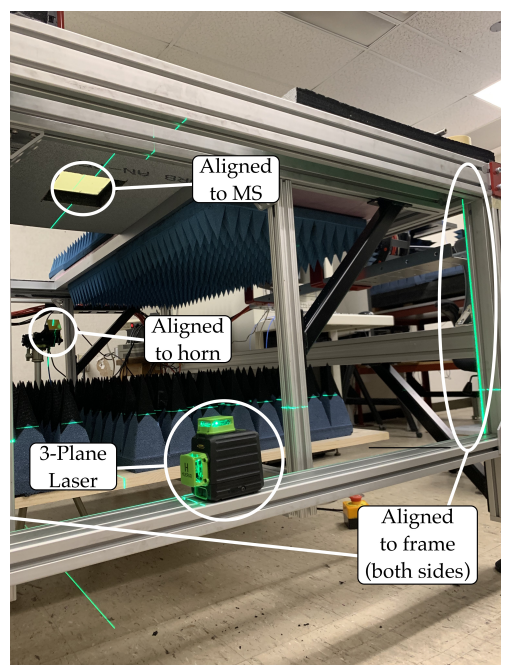

(a) Alignment

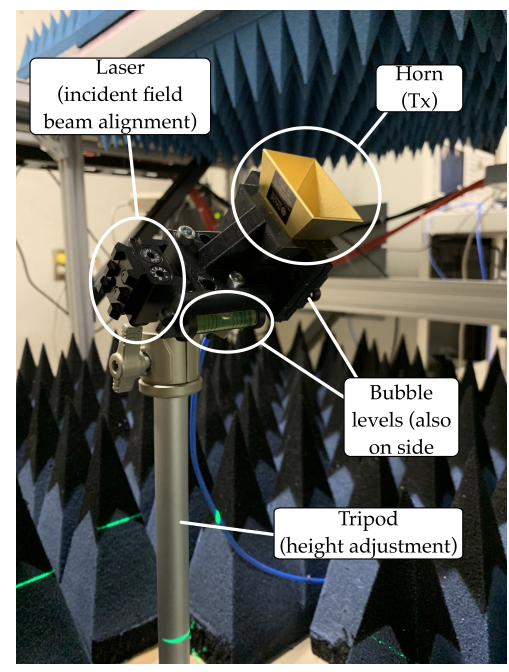

(b) Positioning of Fixed Tx Antenna

Figure 5.4 Alignment of the antennas and MS, primarily using a 3-plane laser. 


\subsubsection{Graphical User Interface (GUI)}

Finally, the custom Python software automates the measurements. I created a user-friendly graphical user interface (GUI), shown in Figure 5.5, which allows for configuring the VNA and DMC, and monitoring automated measurements as they run. When a measurement is complete, it is possible to export a CSV file which contains the S-parameters at each spatial coordinate and frequency configured. These can be then post-processed, e.g. with MATLAB.

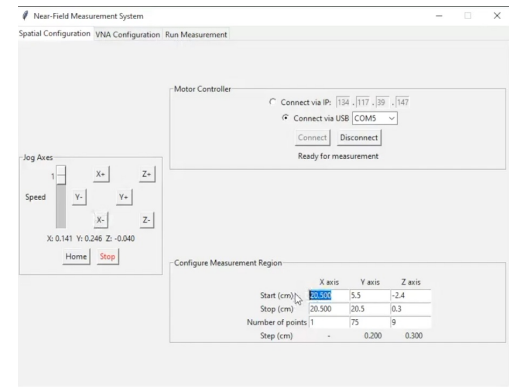

(a) Configuration of DMC

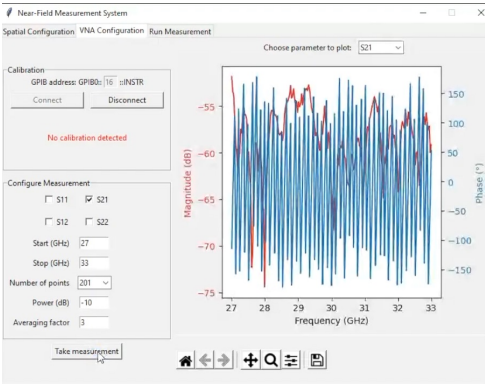

(b) Configuration of VNA

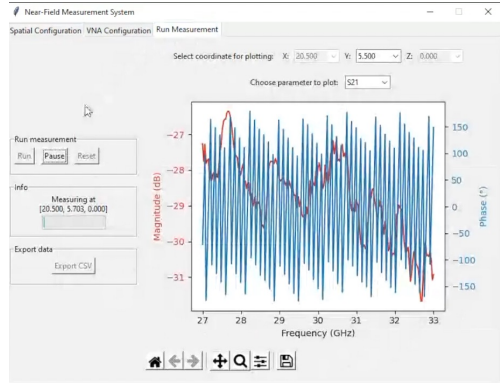

(c) Monitoring an ongoing measurement

Figure 5.5 Shows the three tabs in the custom Python GUI used to run automated NF scans.

\subsection{Fabrication and Measurement of a Periodic Ka-Band MS}

To experimentally demonstrate the Floquet method (Chapter 4), two simple metasurfaces were fabricated, analyzed, and measured using the developed NF system. The designed surfaces do not serve any particular application, but nevertheless provide an opportunity to demonstrate the measurement system. In keeping with the orientation of fields in Chapter 4, the fabricated metasurfaces are designed and anayzed for TE-polarized fields propagating in the $x-z$ plane.

\subsubsection{Unit Cell Design}

A simple unit cell was designed to support tangential electric and magnetic polarization densities, i.e. $\chi_{\mathrm{ee}}^{y y}$ and $\chi_{\mathrm{mm}}^{x x}$, shown in Figure 5.6a. Two identical dogbone-shaped copper dipoles (1/2 ounce copper) are placed on two sides of a thin Rogers RO3010 substrate having a high permittivity. One may expect a $y$-polarized electric dipole supported by symmetric currents flowing in the $\hat{\mathbf{y}}$ direction (producing $\chi_{\mathrm{ee}}^{y y}$ ). By "symmetric", I mean symmetry in the $x-y$ plane, such that the currents have the same direction on each dipole. On the other hand, a $x$-directed magnetic dipole $\left(\chi_{\mathrm{mm}}^{x x}\right)$ is produced with anti-symmetric currents as shown in Figure 5.6b.

Through iterative HFSS simulations of the unit cell, it was optimized to have a magnetic resonance in the Ka-band (around $30 \mathrm{GHz}$ ), with the possibility of tuning using the length of the dogbones, $l$. The extracted susceptibilities are shown in Figure 5.6c for three different values of $l$, with the mag- 
netic resonance clearly visible as a Lorentzian profile of $\chi_{\mathrm{mm}}^{x x}$. The cell is deeply sub-wavelength $\left(1.5 \mathrm{~mm}=\lambda_{0} / 6.66\right.$ at $30 \mathrm{GHz}$ where $\left.\lambda_{0}=10 \mathrm{~mm}\right)$, and is limited by fabrication tolerances which were considered when iteratively designing the dogbone dimensions. The thickness is also deeply sub-wavelength, with an effective thickness $\sqrt{\epsilon_{r}} d=0.43 \mathrm{~mm}$.

While Figure 5.6c shows the susceptibilities at three different values of $l$, the extraction was performed with a more dense sampling of $l$, at the design frequency of $30 \mathrm{GHz}$, to produce the look-up graph in Figure 5.6d. This could subsequently be used to determine the susceptibility at a given value of $l$, for a periodic MS where $l$ is modulated.

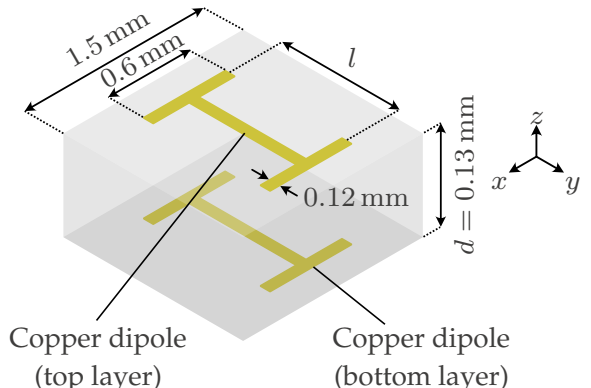

(a) Unit cell

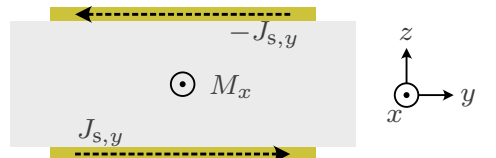

(b) Depiction of magnetic dipole
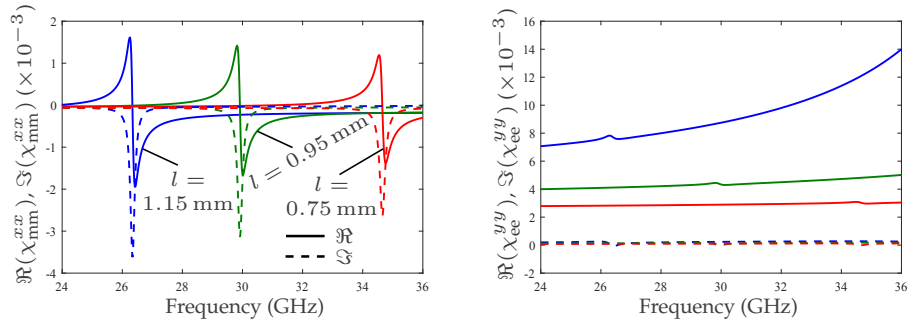

(c) Susceptibilities, with different $l$
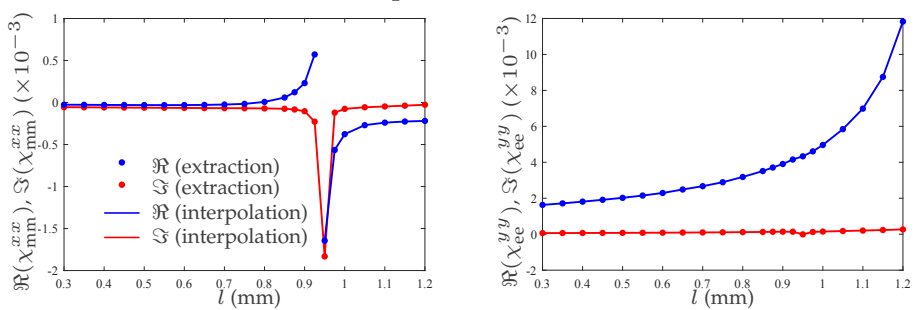

(d) Susceptibilities, at $30 \mathrm{GHz}$

Figure 5.6 A two-layer metasurface was designed to have a magnetic resonance in the Ka-band. (a) Unit cell with identical metal dog-bones on either side of a Rogers RO3010 substrate $\left(\epsilon_{r}=11.2, \tan \delta=0.0022\right)$. (b) Depiction of formation of $x$-directed magnetic dipole $\left(M_{x}\right)$, with antisymmetric $y$-directed surface currents on opposite dipoles $\left( \pm J_{\mathrm{s}, y}\right)$. (c) Extracted susceptibilities for three different values of $l$. (d) Susceptibilities for extractions at all $l$ values, at the design frequency.

\subsubsection{Periodic MS Design}

Two different periodic modulations of $l$ were considered. As the developed Floquet method assumes uniformity in the $y$-direction, the modulation is in the $x$-direction.

1. A "square wave" modulation where $l$ alternates between $l=0.3 \mathrm{~mm}$ and $l=0.95 \mathrm{~mm}$, as shown in Figure 5.7b

2. A "triangle wave" modulation where $l$ varies linearly between throughs and peaks at $l=$ $0.5 \mathrm{~mm}$ and $l=1.2 \mathrm{~mm}$, respectively, as shown in Figure $5.8 \mathrm{~b}$

These plots show the profile of $l$ over a single period $(45 \mathrm{~mm})$, which was selected arbitrarily and consists of 30 unit cells. The continuous profile (solid line) of $l$ was discretized (black points) for the realization using the designed unit cell. The fabricated metasurface for the "square wave" 
modulation is shown in Figure 5.7a, and it has finite dimensions $L_{x}=180 \mathrm{~mm}$ (4 periods) and $L_{y}=127.5 \mathrm{~mm}$ (85 cells). The fabricated metasurface for the "triangle wave" modulation has the same dimensions and is shown in Figure 5.8a.

For these modulations, the respective susceptibilities were found from Figure 5.6d, producing the susceptibility profiles in Figures 5.7c and 5.8c.

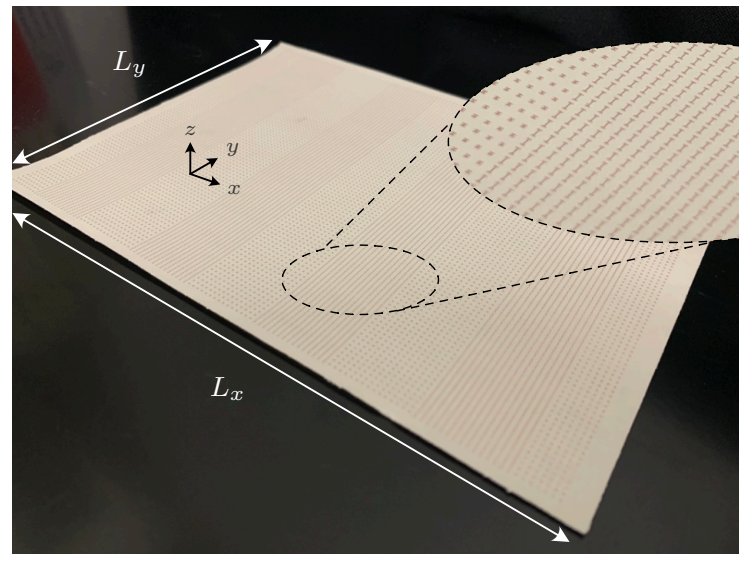

(a) Fabricated MS

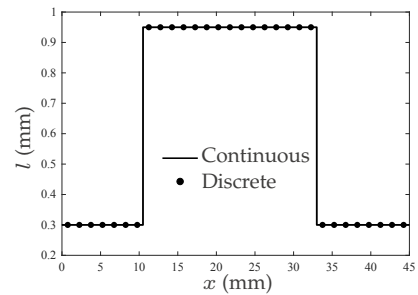

(b) Modulation of $l$

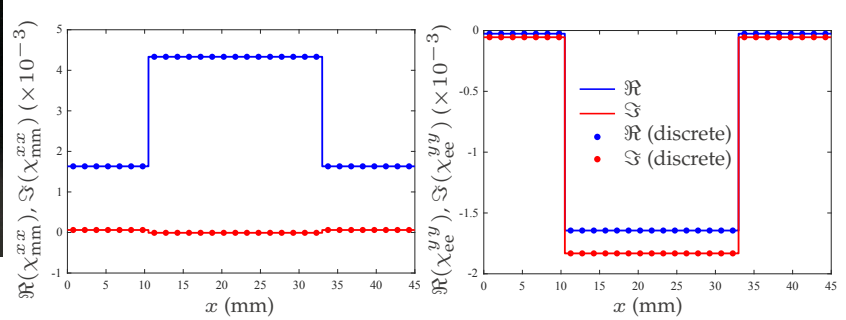

(c) Modulation of susceptibilities

Figure 5.7 A MS was fabricated using a periodic "square wave" modulation of $l$. (a) Fabricated MS. (b) Profile of $l$ over a single period. (c) Profile of susceptilibilities over a single period.

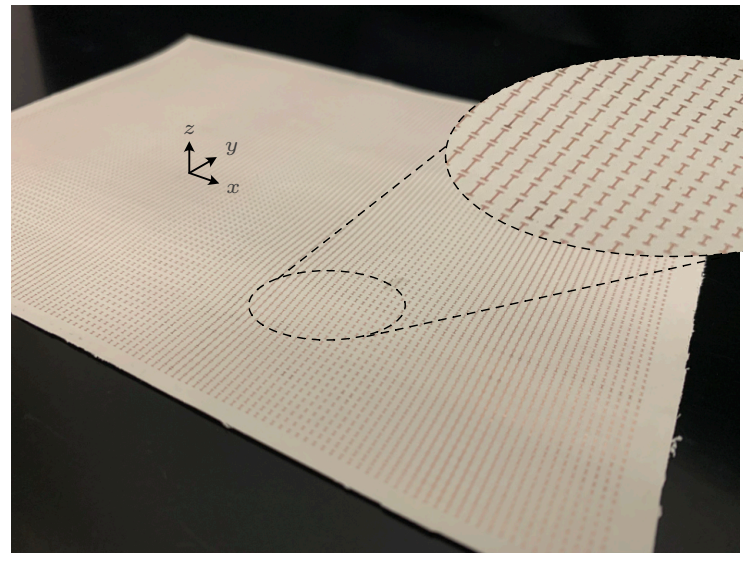

(a) Fabricated MS

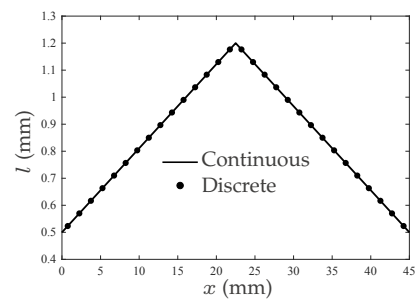

(b) Modulation of $l$

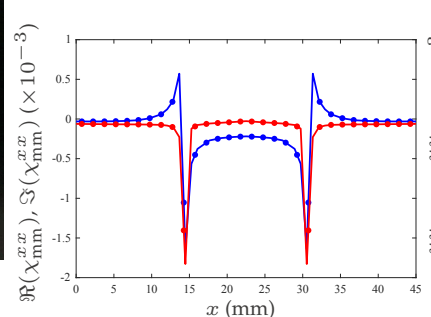

(c) Modulation of susceptibilities

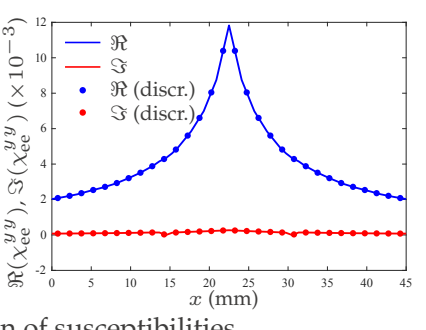

(a) Fabricated MS. (b) Profile of $l$ over Figure 5.8 A MS was fabricated using a periodic "triangle wave" mor
a single period. (c) Profile of susceptilibilities over a single period. 


\subsubsection{Measurement}

The NF measurements were carried out as depicted in Figure 5.1a, with the metasurface at $z=0$ and the horn aperture at $x_{\mathrm{ap}}=0$ and roughly $z_{\mathrm{ap}} \approx-35 \mathrm{~cm}$ with $\theta=0^{\circ}$. The exact position of the horn was not critical, since the actual incident field was measured and considered in the following prediction of the transmitted field, using the Floquet method. Thus, the measurements consisted of two steps:

1. The incident field was measured at $z=0$ with a 1D scan along $x$. For this measurement, the MS is not present so the incident field $\mathbf{E}_{\mathrm{i}}$ was directly measured.

2. Without disturbing the horn antenna, the MS was installed and the transmitted fields $\mathbf{E}_{\mathrm{t}}$ were measured in the $x-z$ plane with a $2 \mathrm{D}$ scan.

In doing these measurements, there were no calibrations or corrections applied to the VNA; it was essentially used as a source and power meter.

First, the incident fields from Step \#1 are shown in Figure 5.9, after normalization, and probed at intervals of $\Delta x=2 \mathrm{~mm}$ (black points). As expected for a normally-oriented horn, the pattern is nearly symmetrical and can be modelled as a Gaussian beam [68]. ${ }^{1}$ There is some noise in the measured points, and I have fitted a Gaussian profile (red line) for use in the Floquet analysis (and for a BEM simulation). The plotted red line is in fact the summation of the plane wave expansion of the Gaussian profile, using the method from Section 4.4.3, which allows the Floquet method to be applied for this aperiodic incident field.
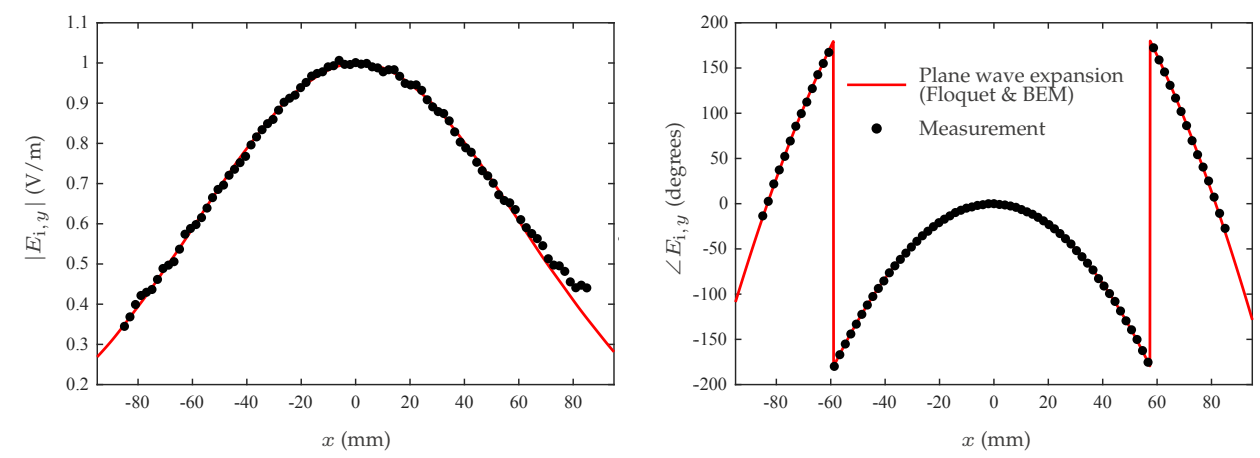

Figure 5.9 Incident field.

Next, the 2D measured transmitted fields for the square-wave modulation are in Figure 5.10a. The field probing intervals were $\Delta x=2 \mathrm{~mm}$ and $\Delta z=4.8 \mathrm{~mm}$, for the $x$ and $z$ directions, respectively. The plotted fields are scaled by the same factor as for in the incident fields in Figure 5.9; that is, the two plots are consistent.

\footnotetext{
${ }^{1}$ This is discussed in Section 5.4.1
} 
The transmitted fields were also predicted using the Floquet method and BEM, and also shown in Figure 5.10a. These show very good agreement, and reasonable agreement with the measurements. The fields are also plotted at an observation plane at $z=20 \mathrm{~mm}$ in Figure 5.10b, with reasonable agreement for the phase as well. It is worth reiterating that no magnitude or phase normalizations have been performed to achieve this agreement, other than setting the peak amplitude of the incident field to $1 \mathrm{~V} / \mathrm{m}$ in Figure 5.9.

However, there is clearly some error between the predicted fields (Floquet/BEM) and the measured fields. In particular, it appears that the measured fields "smooth out" the peaks of the predicted fields. There are three main sources of error, which can account for this. Firstly, the Rx waveguide probe antenna has a finite width of $9.1 \mathrm{~mm}$ in the $x$-direction, which is expected to produce an averaging of the fields. ${ }^{2}$ Secondly, no compensation has been performed for the Rx antenna, which may have coupling to the MS, which disturbs its behaviour. Lastly, the analysis is 2-D, while the measurements in fact are 3-D even though the MS is approximately uniform in the $y$-direction. That is, the fields generated by the horn antenna are 3-D Gaussian beams rather that 2-D Gaussian beams, which propagate differently. Nevertheless, even with these approximations, there is reasonable agreement for this demonstration of the NF system.

\footnotetext{
${ }^{2}$ The waveguide probe, Eravent model SAP-28-R2, was selected as a standard available model.
} 

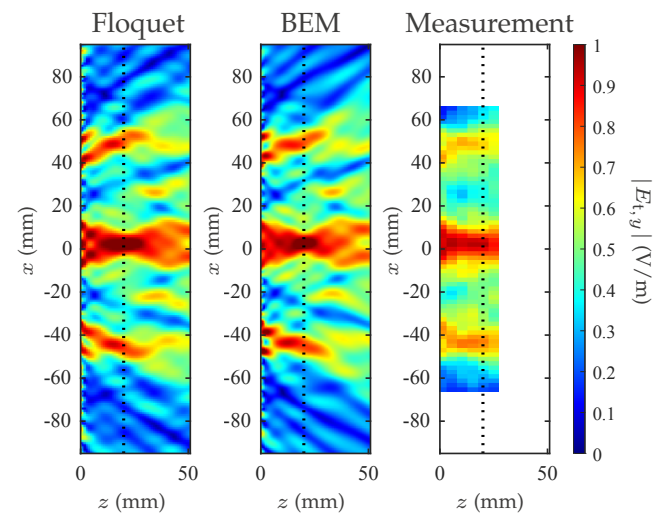

(a) Transmitted field magnitude in $x-z$ plane
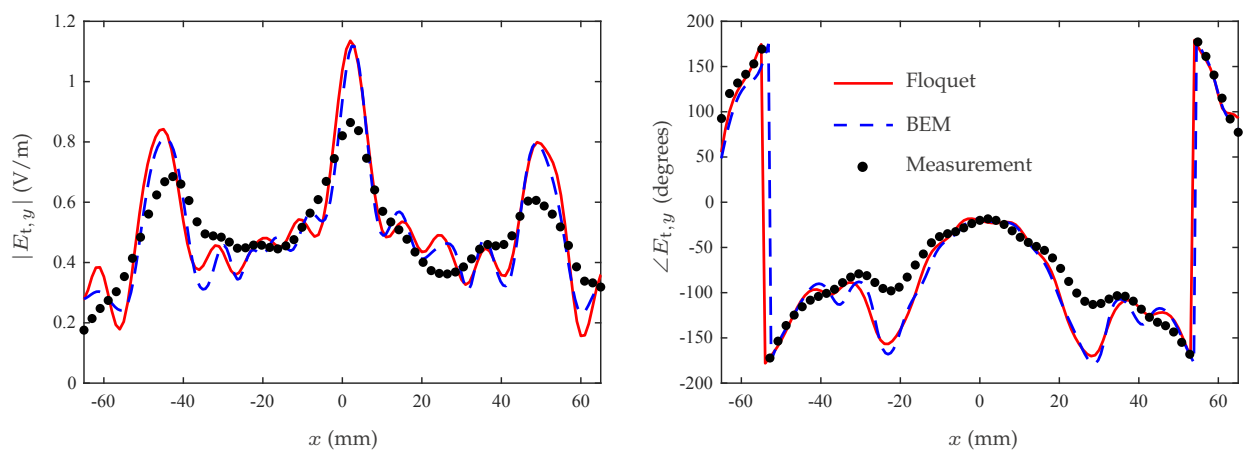

(b) Transmitted field at $z=20 \mathrm{~mm}$ [dashed line in (a)]

Figure 5.10 Transmitted fields from the MS shown in Figure 5.7 with square wave modulation, using the incident field from Figure 5.9.

Next, the MS with triangular modulation was measured and analyzed in a similar fashion with the results in Figure 5.11. The same incident field was used (i.e. the Tx antenna was not disturbed).

Since the modulation is smooth in this case, we might expect that the aforementioned Rx probe averaging might have less effect in the agreement between the Floquet analysis and the measurements. This is in fact the case, and better agreement is observed in the amplitude in Figure 5.11b, while the phase has very good agreement. 


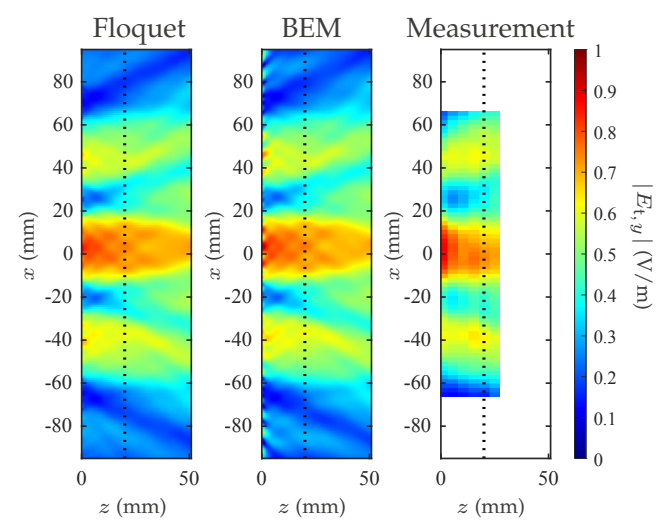

(a) Transmitted field magnitude in $x-z$ plane
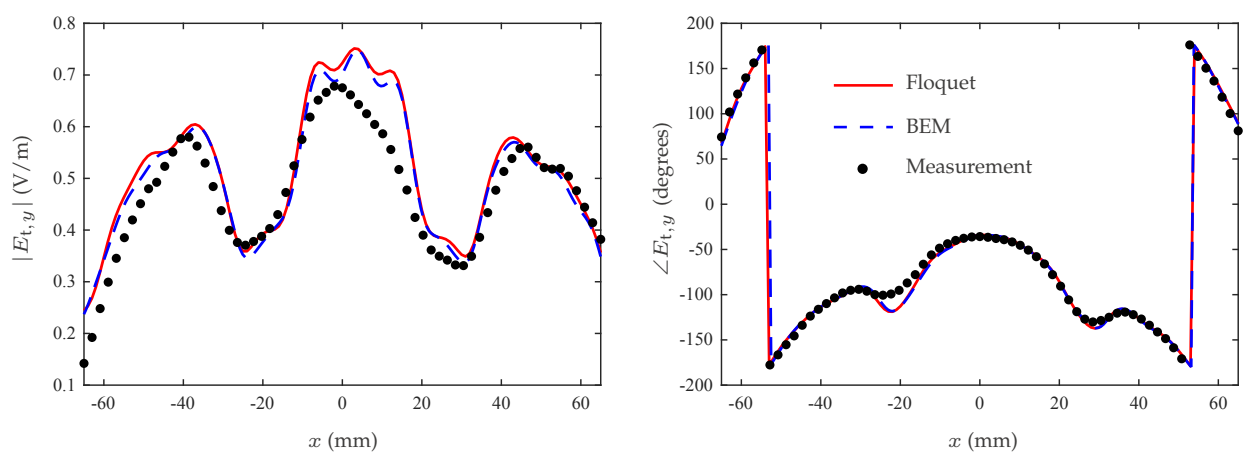

(b) Transmitted field at $z=20 \mathrm{~mm}$ [dashed line in (a)]

Figure 5.11 Transmitted fields from the MS shown in Figure 5.8 with triangle wave modulation, using the incident field from Figure 5.9.

\subsection{Incident Field Reconstruction using a Single Horn Antenna}

It is quickly apparent that the system in Figure 5.1a does not provide much flexibility for controlling the incident field. The designer is limited to using the specific Tx antennas which are available to them, and configuring the orientation and position of the Tx antenna. This is dominantly due to expensive horn antennas used in these systems in practice, where usage of multiple Tx antennas is not always possible and is not cost-effective. Thus, it may be that it is not possible to experimentally produce the exact incident fields that were used in simulation, and for which the MS is originally designed. For example, consider a MS that was designed for a normally incident plane wave. Using a rectangular horn antenna for illumination, it is not possible to produce an ideal plane wave, with both uniform phase and amplitude, as I will show. While it is possible to approximate a plane wave by moving the horn antenna far away from the MS, this comes at the expense of losing much of the incident power, affecting the signal-to-noise ratio and introducing undesired effects due to non-uniform phase distribution across the MS. One approach which has been taken specifically to generate a flat phase is the use of a lens placed between the illuminating 
horn and the MS $[69,70,58]$. This is based on the quasi-optical approximation of the field generated by the horn being a Gaussian beam [68]. In this case, the system can be analyzed within the framework of paraxial optics which can be used to design a lens which produces a beam waist (and hence a constant phase) at the location of the MS [71]. However, the typical spot-size generated using this lensed system is small, of the order of few centimeters for a typical Ka-band system, for instance, which is not sufficient to characterize larger sized MSs (typically several tens of wavelengths), beyond which the phase flatness is significantly degraded. This greatly limits the physical area that can be field scanned. In addition, a quasi-optical approach of modeling the horn field as a Gaussian beam reveals the inherent trade-off present - as the phase and the amplitude uniformity cannot be optimized at the same time. One can form this conclusion directly from the formulation for the Gaussian beam which has a uniform phase profile at the waist where the spot size is smallest and the magnitude variation sharpest.

In this section, I propose a novel technique based on just a single Tx antenna, which does not require additional components such as lenses, and provides flexibility in shaping the incident field including a flat uniform phase across a large physical area. The method involves multiple separate experiments with different incident and scattered fields, which are subsequently combined using superposition to produce the desired incident and scattered fields. The application of the superposition principle assumes a linear system, which is the case for most MSs, including both linear time-invariant (LTI) and linear time-variant (LTV) MSs (non-linear MSs are notably excepted). I will numerically demonstrate the method using an integral equation (IE) simulator [18], while the same procedure can be carried out in a laboratory setting.

First in Section 5.4.1, I show how the field generated by a rectangular horn can be modelled as a Gaussian beam, which provides a convenient analytical model. Using this model, in Section 5.4.2 I show how a particular incident field-I use an example of a normally-incident plane wave-can be generated by the fields from multiple horns in different configurations. Finally, in Section 5.4.3 I apply this incident field to a MS which has been designed for a normally-incident plane wave, showing that it produces the correct scattered field while illumination with a single horn does not.

\subsubsection{Practical Metasurface Illumination}

I will consider the incident fields generated by the Eravant model SAR-2013-28-S2 rectangular horn antenna, which functions in the $26.5 \mathrm{GHz}$ to $40 \mathrm{GHz}$ band. Fig. 5.12a shows the electric field profile in the $H$ plane $(x-z$ plane), simulated using the full-wave Ansys HFSS simulator at $f=30 \mathrm{GHz}$ (simulation model inset in top right). For the simulation, the aperture of the horn is placed at $\left(x_{\mathrm{ap}}, z_{\mathrm{ap}}\right)=(0,0)$. I see that the horn produces curved phase-fronts, with the fields plotted along an observation line at $z=40 \mathrm{~cm}$ in Fig. 5.12c for closer examination. There is significant curvature of the phase: along the observation line at a distance of $x=7.5 \mathrm{~cm}$ from the peak, the phase has decreased by $230^{\circ}$ while the amplitude has reduced to $72 \%$. This is a significant deviation from a flat phase front of a uniform plane-wave, for instance, which is typically used in various MS 
designs.

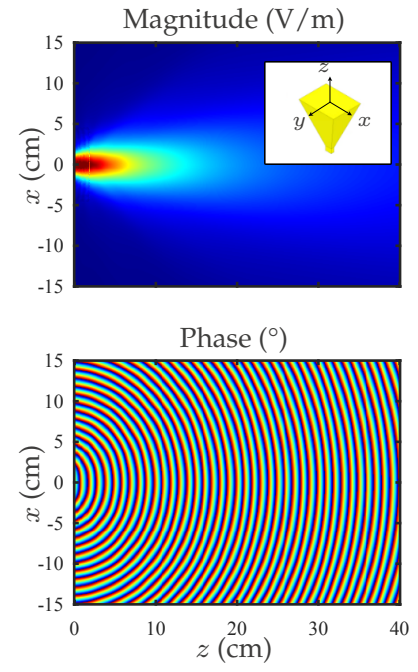

(a) Full-wave horn simulation (HFSS)

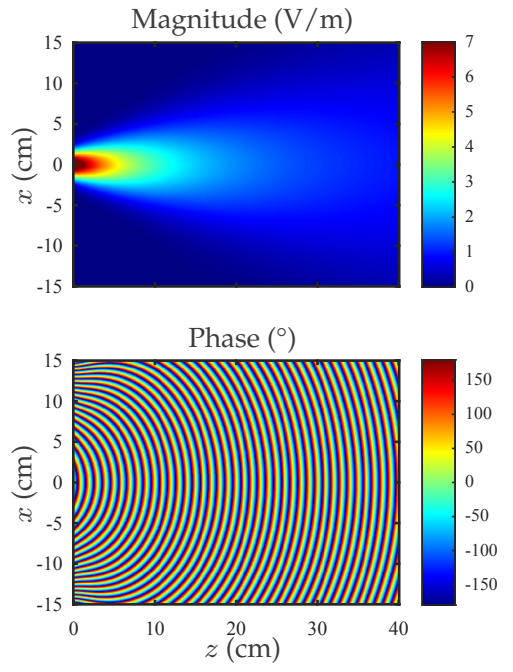

(b) Gaussian beam approximation

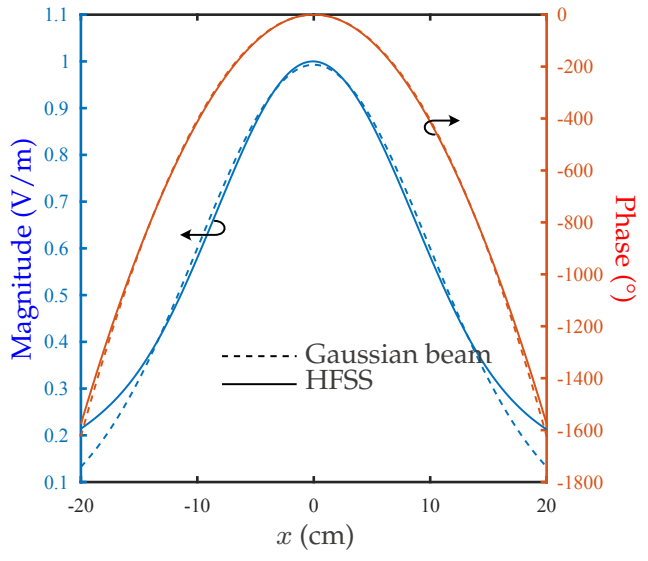

(c) Comparison at $z=40 \mathrm{~cm}$

Figure 5.12 The Eravant SAR-2013-28-S2 horn antenna was simulated using HFSS, and is well-approximated using a Gaussian beam having the parameters $w_{0}=1.00 \mathrm{~mm}, x_{\mathrm{bw}}=0 \mathrm{~cm}$, and $z_{\mathrm{bw}}=-4.14 \mathrm{~cm}$.

For the method I will present, in order to generate desired incident fields using a specific horn antenna fields, it is useful to prepare an approximate analytical form for the field generated by the horn. One possibility is using a Gaussian beam, which has been called quasi-optical [68]. A Gaussian beam is a solution to the paraxial-wave equation, which assumes a slowly-varying amplitude in the direction of propagation. In the $H$-plane $(y=0)$, a Gaussian beam with TE polarization has the form [72]

$$
\mathbf{E}(x, z)=A \frac{w_{0}}{w\left(z^{\prime}\right)} \exp \left[-\frac{\left(x^{\prime}\right)^{2}}{w^{2}\left(z^{\prime}\right)}-j k z^{\prime}+\frac{-j k\left(x^{\prime}\right)^{2}}{2\left(z^{\prime}+\frac{z_{R}^{2}}{z^{\prime}}\right)}-j \arctan \left(\frac{z^{\prime}}{z_{R}}\right)\right] \hat{\mathbf{y}}
$$

where $w\left(z^{\prime}\right)=w_{0} \sqrt{1+\left(z^{\prime}\right)^{2} / z_{R}^{2}}$ is the radius of the beam spot (at which the amplitude is $A / e$ ), $w_{0}$ is beam waist, $z_{R}=\pi w_{0}^{2} / \lambda$ is known as the Raleigh length, $k=2 \pi / \lambda$, and $\lambda$ is the wavelength. Furthermore,

$$
\left[\begin{array}{l}
x^{\prime} \\
z^{\prime}
\end{array}\right]=\left[\begin{array}{cc}
\cos \theta & -\sin \theta \\
\sin \theta & \cos \theta
\end{array}\right]\left[\begin{array}{l}
x-x_{\mathrm{bw}} \\
z-z_{\mathrm{bw}}
\end{array}\right]
$$

allows for displacing beam waist $\left(x_{\mathrm{bw}}, z_{\mathrm{bw}}\right)$ and rotating the beam by an angle $\theta$ about the beam waist.

To determine appropriate parameters to model the horn using (5.1), a numerical fitting was performed, from which it was found that $w_{0}=1.00 \mathrm{~mm}$ and $z_{\mathrm{bw}}=z_{\mathrm{ap}}-4.14 \mathrm{~cm}$ (obviously, $x_{\mathrm{bw}}=$ $0 \mathrm{~cm}$ ). In other words, the beam waist is $4.14 \mathrm{~cm}$ behind the aperture of the horn. The correspond- 
ing field is plotted in Fig. 5.12b, showing a good match to the horn field and substantiating the quasi-optical approach. From Fig. 5.12c, I note a discrepancy past $|x|>13.5 \mathrm{~cm}$. This error could be reduced by including higher-order Hermite-Gaussian modes in the model [72]. Alternatively, it is also possible to use a circular horn instead of a rectangular one, as the former provides greater coupling to the fundamental Gaussian mode $[70,68]$. However, the accuracy within the noted region for the rectangular horn is sufficient for our demonstration. Thus, a Gaussian beam provides a simple, yet accurate model for the horn, which will be used to construct desired incident fields.

\subsubsection{Construction of an Arbitrary Incident Field}

To generate an arbitrary incident field, I can use a superposition of the fields generated by the horn for $N$ different configurations of the horn, corresponding to $N$ near field scans to be performed. In each of these experiments, the position of the beam waist $\left(x_{\mathrm{bw}, n}, z_{\mathrm{bw}, n}\right)$ and rotation $\theta_{n}$ can be adjusted, producing a different incident $\mathbf{E}_{\mathrm{i}, n}$ field and corresponding transmitted field $\mathbf{E}_{\mathrm{t}, n}$. With the surface representing a linear time-invariant (LTI) system, the fields can be superimposed with arbitrary complex weights $A_{n}$ to produce the fields

$$
\mathbf{E}_{\mathrm{a}}\left(\left\{p_{n}\right\}, x, z\right)=\sum_{n=1}^{N} A_{n} \mathbf{E}_{\mathrm{a}, n}\left(p_{n}, x, z\right)
$$

with $\mathrm{a}=(\mathrm{i}, \mathrm{t}), p_{n}=\left\{x_{\mathrm{bw}, n}, z_{\mathrm{bw}, n}, \theta_{n}, A_{n}\right\}$ corresponding to a configuration of the horn, and $\left\{p_{n}\right\}$ $(n=[1, N])$ being the set of configurations. Experimentally, this can be realized by performing a near-field scan $N$ times with the given horn orientations, and subsequently numerically postprocessing to find the total fields (incident or transmitted).

Now, the question is: how to select $\left\{p_{n}\right\}$ such that $\mathbf{E}_{\mathrm{i}}$ is the desired incident field at the plane of the MS, which I take to be at $z=z_{\text {ms }}$ perpendicular to the $z$ axis? It has been shown in the literature that certain fields can be rigorously expanded as Gaussian beams. For example, in [73] a method is presented for expanding a plane wave into a set of Gaussian beams. I will however use numerical optimization using a genetic algorithm. Along with simplicity, this also allows an arbitrary incident field $\mathbf{E}_{\mathrm{i}}\left(x, z_{\mathrm{ms}}\right)$ to be approximated, using an appropriate cost function.

To carry out the optimization, the cost function can be defined as

$$
\operatorname{cost}\left(\left\{p_{n}\right\}\right)=\left[\frac{1}{\Delta x} \int_{x_{0}}^{x_{0}+\Delta x}\left|\mathbf{E}_{\mathrm{i}}\left(\left\{p_{n}\right\}, x, z_{\mathrm{ms}}\right)-\mathbf{E}_{\mathrm{i}, \text { spec }}\left(x, z_{\mathrm{ms}}\right)\right|^{2} d x\right]^{1 / 2}
$$

where the error from the desired field $\mathbf{E}_{\mathrm{i}, \mathrm{spec}}\left(x, z_{\mathrm{ms}}\right)$ is integrated over the region of interest where the MS is to be placed, $x=\left[x_{0}, x_{0}+\Delta x\right]$. Note that this ensures both the amplitude and phase are as desired.

As an example, I will generate $\left\{p_{n}\right\}$ such that the desired field has a flat phase and amplitude over $x=[-7.5 \mathrm{~cm}, 7.5 \mathrm{~cm}]$; i.e, a plane wave. I will set $\theta_{n}=0^{\circ}, z_{\mathrm{ms}}=0 \mathrm{~cm}$ and $z_{\mathrm{ap}, n}=-40 \mathrm{~cm}$ (i.e. 
$\left.z_{\mathrm{bw}, n}=-44.14 \mathrm{~cm}\right)$. In addition, the positions, $x_{\mathrm{bw}, n}$, are set at uniform intervals, resembling an antenna array. This leaves $A_{n}$ as parameters for the optimization, which was performed using a genetic algorithm in MATLAB. Using $N=8$, I am able to produce the incident field in Fig. 5.13, using the parameters in Table 5.1. The amplitude has less than $4 \%$ amplitude error and $20^{\circ}$ phase error over the specified $15 \mathrm{~cm}$ span. If a better approximation is required, the number of configurations $N$ can be increased, at the cost of more near-field scans to complete. Note that while this field uses $N=8$ horn configurations, only four need to be experimentally performed if the MS is also symmetrical.

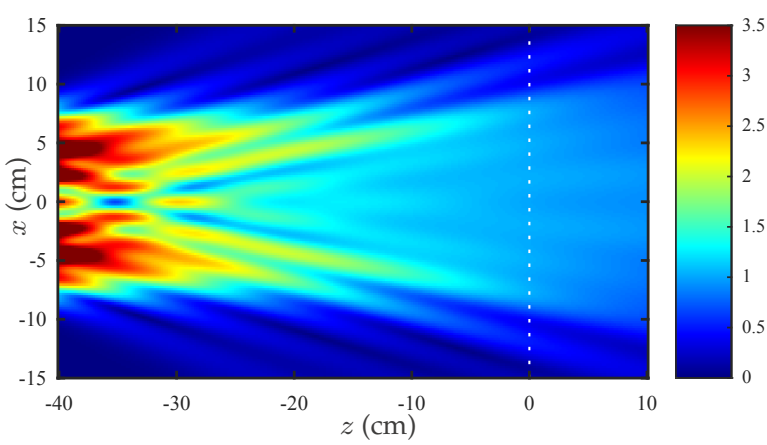

(a) Magnitude of total fields $(\mathrm{V} / \mathrm{m})$

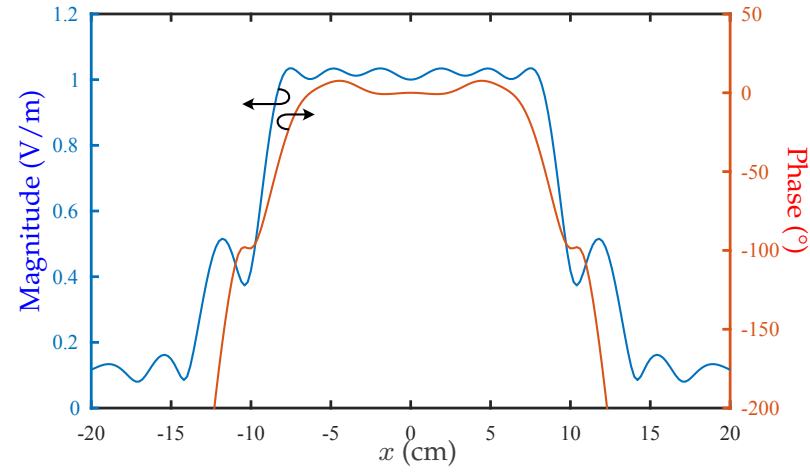

(b) Field at $z=0 \mathrm{~cm}$

Figure 5.13 An incident field $\mathbf{E}_{\mathrm{i}}\left(\left\{p_{n}\right\}, x, z\right)$ generated with the superposition of $N=8$ illuminations, with the objective of uniform amplitude and phase (i.e. normally incident plane wave) over $|x|<7.5 \mathrm{~cm}$.

Table 5.1 Optimized horn configurations for uniform amplitude and phase over $|x|<7.5 \mathrm{~cm}$, using $N=8$ illuminations.

\begin{tabular}{ccc}
\hline$n$ & $x_{\mathrm{ap}, n}(\mathrm{~cm})$ & $A_{n}$ \\
\hline 1,2 & \pm 7.50 & $2.39 e^{-j 0.63}$ \\
3,4 & \pm 5.36 & $5.79 e^{-j 0.20}$ \\
5,6 & \pm 3.21 & $6.90 e^{j 0.40}$ \\
7,8 & \pm 1.07 & $3.72 e^{j 0.45}$ \\
\hline
\end{tabular}

\subsubsection{Example: A Parabolic Surface}

To demonstrate the utility of the plane wave approximation using superposition, I numerically consider the measurement of a MS synthesized for a practical field transformation. The surface is first designed with the assumption of plane wave incidence; I choose to characterize the required MS in terms of surface susceptibilities. Subsequently, I use the synthesized susceptibilities in the integral equation (IE) simulator in [18], with incident fields corresponding to (1) the ideal fields used for the design, (2) a single horn antenna, and (3) the superposition of horn fields determined in Section 5.4.2. 
Firstly, I will design the MS to focus a normally incident plane wave to a focal point $\mathbf{r}_{\mathrm{f}}=\left(x_{\mathrm{f}}, z_{\mathrm{f}}\right)$ i.e. a flat focussing lens in transmission. I desire the transmitted field to be a cylindrical wave, given at the point $\mathbf{r}=(x, z)$ by

$$
\begin{aligned}
& E_{\mathrm{t}, y}(\mathbf{r})=E_{0} \frac{H_{0}^{(1)}\left(k\left|\mathbf{r}-\mathbf{r}_{\mathrm{f}}\right|\right)}{H_{0}^{(1)}\left(k\left|\mathbf{r}_{\mathrm{f}}\right|\right)} \\
& H_{\mathrm{t}, x}(\mathbf{r})=E_{0} \frac{j\left(z-z_{\mathrm{f}}\right) H_{1}^{(1)}\left(k\left|\mathbf{r}-\mathbf{r}_{\mathrm{f}}\right|\right)}{\eta|\mathbf{r}| H_{0}^{(1)}\left(k\left|\mathbf{r}_{\mathrm{f}}\right|\right)} \\
& H_{\mathrm{t}, z}(\mathbf{r})=-E_{0} \frac{j\left(x-x_{\mathrm{f}}\right) H_{1}^{(1)}\left(k\left|\mathbf{r}-\mathbf{r}_{\mathrm{f}}\right|\right)}{\eta|\mathbf{r}| H_{0}^{(1)}\left(k\left|\mathbf{r}_{\mathrm{f}}\right|\right)}
\end{aligned}
$$

where $H_{\{0,1\}}^{(1)}$ are Hankel functions of the first kind, representing inward propagating cylindrical waves, and the fields have been scaled such that $E_{\mathrm{t}, y}(0,0)=E_{0}$. Meanwhile, the incident field is a normally incident plane wave normalized such that $E_{\mathrm{i}, y}=E_{0}$ and $H_{\mathrm{i}, x}=-E_{0} / \eta$, and furthermore I desire there to be no reflection, i.e. a matched lens. For this field transformation, suitable susceptibilities are [74]

$$
\begin{aligned}
\chi_{\mathrm{ee}}^{y y}(x) & =\frac{-j}{\pi \epsilon f}\left(\frac{H_{\mathrm{t}, x}(x, 0)-H_{\mathrm{i}, x}(x, 0)}{E_{\mathrm{t}, y}(x, 0)+E_{\mathrm{i}, y}(x, 0)}\right) \\
\chi_{\mathrm{mm}}^{x x}(x) & =\frac{-j}{\pi \mu f}\left(\frac{E_{\mathrm{t}, y}(x, 0)-E_{\mathrm{i}, y}(x, 0)}{H_{\mathrm{t}, x}(x, 0)+H_{\mathrm{i}, x}(x, 0)}\right)
\end{aligned}
$$

Fig. 5.14a shows these synthesized susceptibilities at $f=30 \mathrm{GHz}$, with $\left(x_{\mathrm{f}}, z_{\mathrm{f}}\right)=(0 \mathrm{~cm}, 10 \mathrm{~cm})$. Note that $\Im\left\{\chi_{\mathrm{ee}}^{y y}(x)\right\}<0$ and $\Im\left\{\chi_{\mathrm{mm}}^{x x}(x)\right\}<0$, which indicates that the required MS is passive.

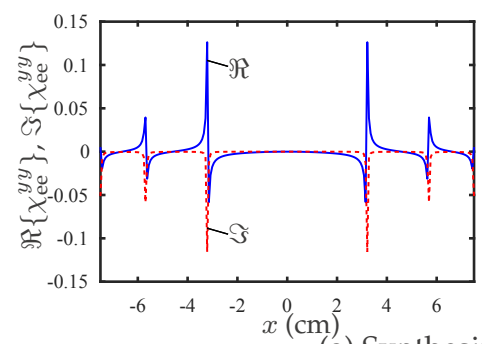

(a) Synthesized susceptibilities

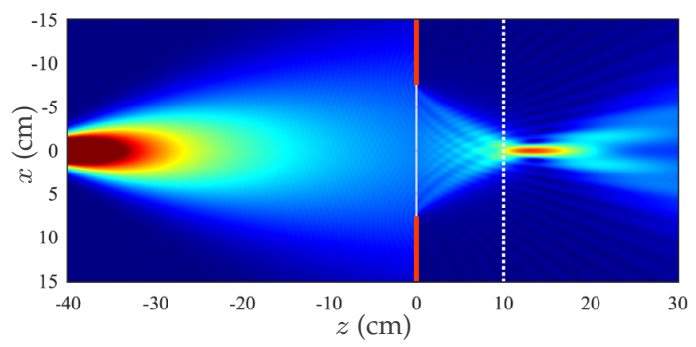

(c) Horn illumination
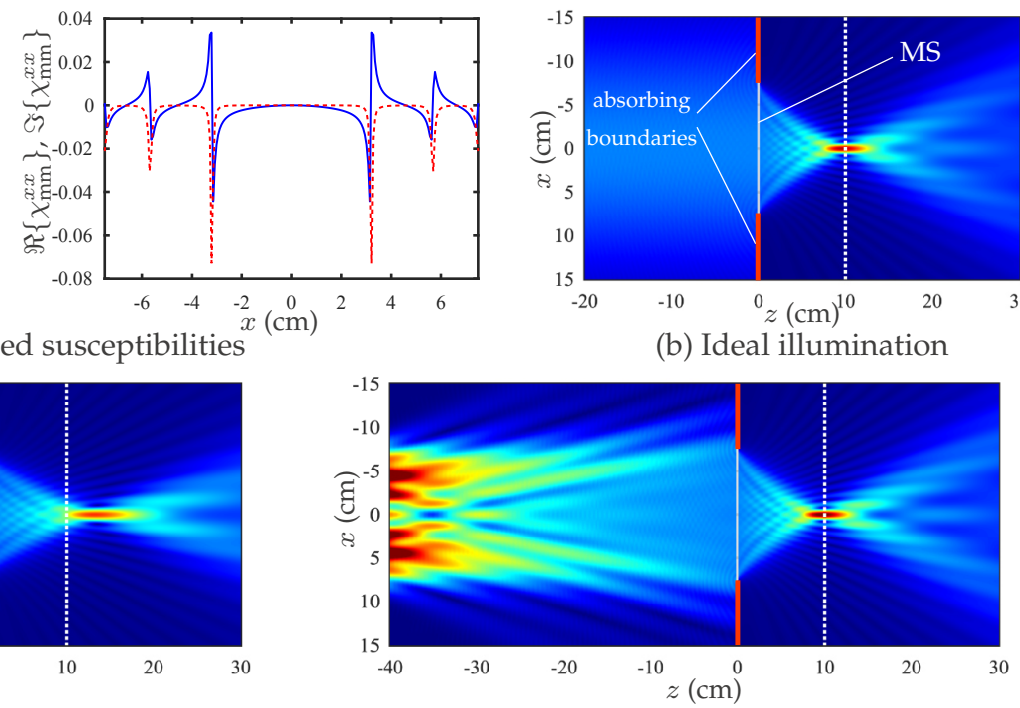

(d) Superposition, $N=8$

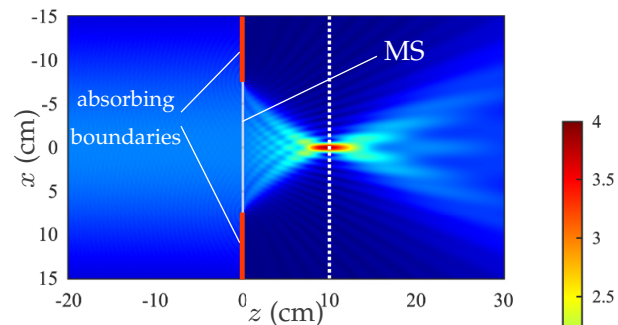

(b) Ideal illumination

Figure 5.14 A focusing MS was designed and then illuminated with three different incident fields in (b-d). 
Next, the MS is illuminated using a field having $\left|E_{\mathrm{i}}\right| \approx 1$ and $\angle E=0^{\circ}$; i.e., what the MS was designed for. (More precisely, a Gaussian beam with $w_{0}=15 \mathrm{~cm}$ and beam waist at $z=0$ is used, with negligible error compared to a plane wave over the length of the MS.) This is shown in Fig. 5.14b, where a focal spot at $z=10 \mathrm{~cm}$ is observed, as ideally desired. This indicates that susceptibilities in Fig. 5.14a were correctly selected.

However, this illumination is naturally very different from the illumination of a practical horn antenna and thus not possible to achieve in practice. The large phase curvature of the horn antenna by itself is expected to generate significantly different scattered fields as compared to the original MS designed for normally incident plane-wave, as horn fields contain large angular spectrum. To see this more clearly, if the surface is illuminated with the horn from Section 5.4.1, I observe the fields in Fig. 5.14c. Clearly, the focal spot has shifted by several centimeters, which is a significant deviation relative to the wavelength $(\lambda=1 \mathrm{~cm}$ at $30 \mathrm{GHz})$. Observing this result experimentally might be (erroneously) interpreted as an indication that the MS was not designed correctly. However, as I have noted, the horn does not produce the appropriate field for characterizing the MS. This illustration thus highlights the importance of correctly choosing the Tx antenna in the measurement stages for accurate surface characterization.

Next, consider the set of $N=8$ horn configurations from Table 5.1. After superimposing the total fields, the results in Fig. 5.14d is achieved, where the focal spot is now once again observed at $z=10 \mathrm{~cm}$, as desired. Thus, the proposed procedure provides a good approximation of the desired incident field in this case. While I only considered $A_{n}$ and $x_{\mathrm{bw}, n}$ in the optimization for this specific example, it is naturally possible to include $z_{\mathrm{bw}, n}$ and $\theta_{n}$ as well, for potentially more flexibility. Furthermore, it is possible to change the desired field objective in (5.4) for other fields which are not necessarily plane waves.

\subsection{Conclusions \& Future Work}

A NF measurement system was developed, which is capable of automated measurements of the transmitted fields of a MS, from $26.5 \mathrm{GHz}$ to $40 \mathrm{GHz}$. This is expected to be a valuable tool for future research of the Metamaterials and Antennas Research Group at Carleton University. Furhermore, a straightforward but novel technique was proposed for reconstructing arbitrary incident fields for NF measurements, using a single Tx antenna in multiple separate measurements.

The preliminary measurements shown in this chapter do not account for the presence of the $\mathrm{Rx}$ probe antenna, and future work includes compensating for this, or modelling it. Furthermore, the incident field reconstruction method has not yet been experimentally demonstrated, and remains another interesting direction to explore. 


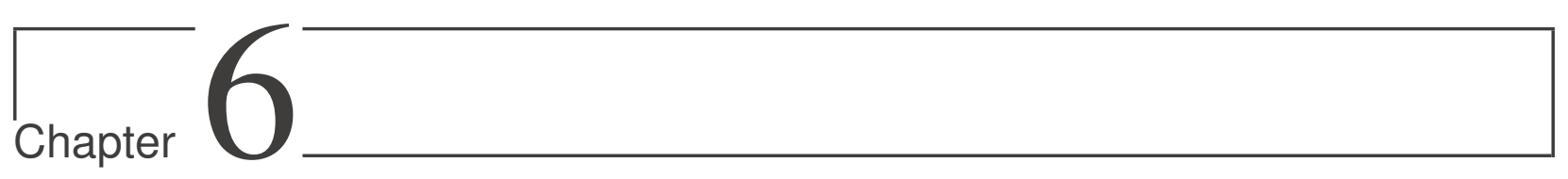

\section{SUMMARY AND FUTURE WORK}

\subsection{Key Conclusions}

This thesis presents a fast and semi-analytical formulation for calculating the scattering from a space-time modulated MS, along with consideration of applicability of the susceptibilities that can be used with it, and experimental methods for characterizing MSs that are fabricated. Firstly, a surface susceptibility model was developed for fully-reflective MSs, using the conventional twosided GSTCs and the HK model for the susceptibilities. The susceptibilities work for both forwards and backwards illumination, behaving as a PEC for the latter, which is important if the surface is finite such that the fields may interact with the reverse side. The bi-anisotropic components play a crucial role in the asymmetric scattering with no transmission. Secondly, a fast and semi-analytical method for calculating the scattered field from a space-time modulated MS was developed. For rigor, the frequency dispersion of the MS is modelled using susceptibilities having Lorentzian frequency profiles, and this was demonstrated to be necessary for accurate modelling, with comparison to FDTD simulations. The method was verified for time-only, space-only, and space-time modulations. In the case of time modulation, frequency generation and non-reciprocity were observed. Lastly, the development of a Ka-band NF measurement system was presented. The system is capable of automated measurements of the transmitted fields of a MS, from $26.5 \mathrm{GHz}$ to $40 \mathrm{GHz}$. This is expected to be a valuable tool for future research of the Metamaterials and Antennas Research Squad at Carleton University. Furthermore, a straightforward but novel technique was proposed for reconstructing arbitrary incident fields for NF measurements, using a single Tx antenna in multiple separate measurements. Together, these contributions form important parts of a methodology for designing, modelling, and experimentally measuring space-time modulated metasurfaces. 


\subsection{Future Work}

Future research on the topics discussed in this thesis could potentially involve the following topics:

1. Chapter 3: An extraction was shown for a unit cell having a tangential electric dipole on top of a ground plane with a dielectric cover layer. As future work, the cell geometry could be made more complex, such as adding a normal polarizability (e.g. vertical dipole on a ground plane), or polarization conversion. Then, is it still possible to have a surface susceptibility representation that works for both forwards and backwards illumination?

2. Chapter 4: The method was presented for TE-polarized fields propagating in the $x-z$ plane, for simplicity. Future work could include extending the approach to arbitrary polarization and propagation. Another direction for future work could be the realization of a unit cell which has temporal modulation at a frequency comparable to the incident field. This would allow frequency mixing resulting in significant frequency conversion.

3. Chapter 5: The preliminary measurements shown in this chapter do not account for the presence of the Rx probe antenna. Compensation for the probe has been considered in other works [67], and this should be included as a post-processing step after using the NF system. Furthermore, the incident field reconstruction method has not yet been experimentally demonstrated, and remains another interesting direction to explore. 


\section{Bibliography}

[1] A. C. Overvig, S. Shrestha, S. C. Malek, M. Lu, A. Stein, C. Zheng, and N. Yu, “Dielectric metasurfaces for complete and independent control of the optical amplitude and phase," Light Sci Appl, vol. 8, no. 1, p. 92, Oct. 2019.

[2] X. Wang, A. Díaz-Rubio, and S. A. Tretyakov, "Independent Control of Multiple Channels in Metasurface Devices," Phys. Rev. Applied, vol. 14, no. 2, p. 024089, Aug. 2020.

[3] N. Yu and F. Capasso, "Flat optics with designer metasurfaces," Nat Mater., vol. 13, Apr. 2014.

[4] A. Lalbakhsh, M. U. Afzal, T. Hayat, K. P. Esselle, and K. Mandal, "All-metal wideband metasurface for near-field transformation of medium-to-high gain electromagnetic sources," Sci Rep, vol. 11, no. 1, p. 9421, May 2021.

[5] E. Björnson, Ö. Özdogan, and E. G. Larsson, "Reconfigurable Intelligent Surfaces: Three Myths and Two Critical Questions," IEEE Commun. Mag., vol. 58, no. 12, pp. 90-96, Dec. 2020.

[6] L. Zhang, X. Q. Chen, S. Liu, Q. Zhang, J. Zhao, J. Y. Dai, G. D. Bai, X. Wan, Q. Cheng, G. Castaldi, V. Galdi, and T. J. Cui, "Space-time-coding digital metasurfaces," Nat. Commun, vol. 9, no. 1, p. 4334, 2018.

[7] S. Taravati and G. V. Eleftheriades, "Generalized Space-Time-Periodic Diffraction Gratings: Theory and Applications," Phys Rev Appl, vol. 12, no. 2, p. 024026, 2019.

[8] — , "Full-Duplex Nonreciprocal Beam Steering by Time-Modulated Phase-Gradient Metasurfaces," Phys Rev Appl, vol. 14, no. 1, p. 014027, 2020.

[9] E. Kuester, M. Mohamed, M. Piket-May, and C. Holloway, "Averaged transition conditions for electromagnetic fields at a metafilm," IEEE Trans Antennas Propag, vol. 51, no. 10, pp. 26412651, Oct. 2003.

[10] C. L. Holloway, A. Dienstfrey, E. F. Kuester, J. F. O’Hara, A. K. Azad, and A. J. Taylor, “A discussion on the interpretation and characterization of metafilms/metasurfaces: The twodimensional equivalent of metamaterials," Metamaterials, vol. 3, no. 2, pp. 100-112, Oct. 2009. 
[11] C. Kittel and P. McEuen, "Crystal Structure," in Introduction to Solid State Physics, 8th ed., Hoboken, USA, 2018.

[12] X. Liu, F. Yang, M. Li, and S. Xu, "Generalized Boundary Conditions in Surface Electromagnetics: Fundamental Theorems and Surface Characterizations," Appl Sci, vol. 9, no. 9, p. 1891, Jan. 2019.

[13] K. Achouri, M. A. Salem, and C. Caloz, “General Metasurface Synthesis Based on Susceptibility Tensors," IEEE Trans Antennas Propag, vol. 63, no. 7, pp. 2977-2991, July 2015.

[14] K. Achouri and O. J. F. Martin, "Angular Scattering Properties of Metasurfaces," IEEE Trans Antennas Propag, vol. 68, no. 1, pp. 432-442, Jan. 2020.

[15] — _ "Fundamental Properties and Classification of Polarization Converting Bianisotropic Metasurfaces," IEEE Trans Antennas Propag, pp. 1-1, 2021.

[16] V. S. Asadchy, A. Díaz-Rubio, and S. A. Tretyakov, "Bianisotropic metasurfaces: Physics and applications," Nanophotonics, vol. 7, no. 6, pp. 1069-1094, June 2018.

[17] V. Tiukuvaara, T. J. Smy, and S. Gupta, "Floquet Analysis of Space-Time Modulated Metasurfaces with Lorentz Dispersion," IEEE Trans Antennas Propag, pp. 1-1, 42021.

[18] T. J. Smy, V. Tiukuvaara, and S. Gupta, "IE-GSTC Metasurface Field Solver using Surface Susceptibility Tensors with Normal Polarizabilities," ArXiv210505875 Phys., May 2021.

[19] J. Y. H. Teo, L. J. Wong, C. Molardi, and P. Genevet, "Controlling electromagnetic fields at boundaries of arbitrary geometries," Phys Rev A, vol. 94, no. 2, pp. 023 820-, 2016.

[20] X. Jia, Y. Vahabzadeh, C. Caloz, and F. Yang, "Synthesis of Spherical Metasurfaces Based on Susceptibility Tensor GSTCs," IEEE Trans. Antennas Propag., vol. 67, no. 4, pp. 2542-2554, 2019.

[21] C. Holloway, M. Mohamed, E. Kuester, and A. Dienstfrey, "Reflection and transmission properties of a metafilm: With an application to a controllable surface composed of resonant particles," IEEE Trans. Electromagn. Compat., vol. 47, no. 4, pp. 853-865, Nov. 2005.

[22] C. L. Holloway, E. F. Kuester, J. A. Gordon, J. O'Hara, J. Booth, and D. R. Smith, “An Overview of the Theory and Applications of Metasurfaces: The Two-Dimensional Equivalents of Metamaterials," IEEE Antenn Propag Mag, vol. 54, no. 2, pp. 10-35, Apr. 2012.

[23] M. Albooyeh, "Electromagnetic Characterization of Metasurfaces," Ph.D. dissertation, Aalto University, 2015.

[24] M. Albooyeh, S. Tretyakov, and C. Simovski, "Electromagnetic characterization of bianisotropic metasurfaces on refractive substrates: General theoretical framework," Ann. Phys., vol. 528, no. 9-10, pp. 721-737, 2016. 
[25] C. L. Holloway, E. F. Kuester, and A. Dienstfrey, “Characterizing Metasurfaces/Metafilms: The Connection Between Surface Susceptibilities and Effective Material Properties," IEEE Antennas Wirel Propag, vol. 10, pp. 1507-1511, 2011.

[26] A. U. Bhobe, "Derivation of equivalent boundary conditions using the homogenization method and their implementation in time-domain electromagnetics techniques," Ph.D., University of Colorado at Boulder, Colorado, USA, 2003.

[27] M. Dehmollaian, Y. Vahabzadeh, K. Achouri, and C. Caloz, "Limitations of the Metasurface Diluted-Slab Model," IEEE J Multiscale Multiphys Comput Tech, vol. 5, pp. 255-264, 2020.

[28] T. B. A. Senior and J. L. Volakis, Approximate Boundary Conditions in Electromagnetics. IET, 1995.

[29] C. Holloway and E. Kuester, "Impedance-type boundary conditions for a periodic interface between a dielectric and a highly conducting medium," IEEE Trans Antennas Propag, vol. 48, no. 10, pp. 1660-1672, Oct. 2000.

[30] C. L. Holloway and E. F. Kuester, "Equivalent boundary conditions for a perfectly conducting periodic surface with a cover layer," Radio Sci, vol. 35, no. 3, pp. 661-681, 2000.

[31] K. Achouri and C. Caloz, Electromagnetic Metasurfaces: Theory and Applications, 1st ed. Hoboken, NJ: Wiley-IEEE Press, May 2021.

[32] S. J. Orfanidis, Electromagnetic Waves and Antennas, 2016.

[33] A. L. Cullen, "A Travelling-Wave Parametric Amplifier," Nature, vol. 181, no. 4605, pp. 332332, 1958.

[34] E. S. Cassedy and A. A. Oliner, "Dispersion relations in time-space periodic media: Part I-Stable interactions," Proceedings of the IEEE, vol. 51, no. 10, pp. 1342-1359, 1963.

[35] H.-T. Chen, A. J. Taylor, and N. Yu, "A review of metasurfaces: Physics and applications," Rep. Prog. Phys., vol. 79, no. 7, p. 076401, 2016.

[36] P. Genevet and F. Capasso, "Holographic optical metasurfaces: A review of current progress," Rep. Prog. Phys., vol. 78, no. 2, p. 024401, 2015.

[37] J. D. Adam, L. E. Davis, G. F. Dionne, E. F. Schloemann, and S. N. Stitzer, "Ferrite devices and materials," IEEE Trans. Microw. Theory Techn., vol. 50, no. 3, pp. 721-737, 2002.

[38] Y. Shi, Z. Yu, and S. Fan, "Limitations of nonlinear optical isolators due to dynamic reciprocity," Nat. Photon, vol. 9, no. 6, pp. 388-392, 2015.

[39] C. Caloz and Z.-L. Deck-Léger, "Spacetime Metamaterials-Part I: General Concepts," IEEE Trans Antennas Propag, vol. 68, no. 3, pp. 1569-1582, Mar. 2020. 
[40] C. Caloz and Z. Deck-Léger, "Spacetime Metamaterials-Part II: Theory and Applications," IEEE Trans Antennas Propag, vol. 68, no. 3, pp. 1583-1598, 2020.

[41] X. Wang, A. Díaz-Rubio, H. Li, S. A. Tretyakov, and A. Alù, “Theory and Design of Multifunctional Space-Time Metasurfaces," Phys Rev Appl, vol. 13, no. 4, p. 044040, 2020.

[42] Y. Hadad, D. L. Sounas, and A. Alu, "Space-time gradient metasurfaces," Phys Rev B, vol. 92, no. 10, p. 100304, 2015.

[43] D. Ramaccia, D. L. Sounas, A. Alù, A. Toscano, and F. Bilotti, "Phase-Induced Frequency Conversion and Doppler Effect With Time-Modulated Metasurfaces," IEEE Trans Antennas Propag, vol. 68, no. 3, pp. 1607-1617, 2020.

[44] S. Taravati, N. Chamanara, and C. Caloz, "Nonreciprocal electromagnetic scattering from a periodically space-time modulated slab and application to a quasisonic isolator," Phys Rev B, vol. 96, no. 16, p. 165144, 2017.

[45] S. Taravati and A. A. Kishk, "Space-Time Modulation: Principles and Applications," IEEE Microw. Mag., vol. 21, no. 4, pp. 30-56, 2020.

[46] L. Zhang, X. Q. Chen, R. W. Shao, J. Y. Dai, Q. Cheng, G. Castaldi, V. Galdi, and T. J. Cui, "Breaking Reciprocity with Space-Time-Coding Digital Metasurfaces," Adv. Mater., vol. 31, no. 41, p. 1904069 , Oct. 2019.

[47] L. Zhang, Z. X. Wang, R. W. Shao, J. L. Shen, X. Q. Chen, X. Wan, Q. Cheng, and T. J. Cui, “Dynamically Realizing Arbitrary Multi-Bit Programmable Phases Using a 2-Bit Time-Domain Coding Metasurface," IEEE Trans. Antennas Propag., vol. 68, no. 4, pp. 2984-2992, 2020.

[48] T. J. Smy and S. Gupta, “Finite-Difference Modeling of Broadband Huygens' Metasurfaces Based on Generalized Sheet Transition Conditions," IEEE Trans Antennas Propag, vol. 65, no. 5, pp. 2566-2577, 2017.

[49] S. A. Stewart, T. J. Smy, and S. Gupta, "Finite-Difference Time-Domain Modeling of Space-Time-Modulated Metasurfaces," IEEE Trans Antennas Propag, vol. 66, no. 1, pp. 281$292,2018$.

[50] T. J. Smy, S. A. Stewart, J. G. N. Rahmeier, and S. Gupta, “FDTD Simulation of Dispersive Metasurfaces With Lorentzian Surface Susceptibilities," IEEE Access, vol. 8, pp. 83 027-83 040, 2020.

[51] Y. Vahabzadeh, N. Chamanara, K. Achouri, and C. Caloz, "Computational Analysis of Metasurfaces," IEEE J Multiscale Multiphys Comput Tech, vol. 3, pp. 37-49, 2018.

[52] S. A. Stewart, S. Moslemi-Tabrizi, T. J. Smy, and S. Gupta, "Scattering Field Solutions of Metasurfaces Based on the Boundary Element Method for Interconnected Regions in 2-D," IEEE Trans Antennas Propag, vol. 67, no. 12, pp. 7487-7495, 2019. 
[53] N. Chamanara, Y. Vahabzadeh, and C. Caloz, "Simultaneous Control of the Spatial and Temporal Spectra of Light With Space-Time Varying Metasurfaces," IEEE Trans Antennas Propag, vol. 67, no. 4, pp. 2430-2441, 2019.

[54] E. Rothwell and M. Cloud, Electromagnetics, 3rd ed. Boca Raton, FL, USA: CRC Press, 2018.

[55] S. Inampudi, M. M. Salary, S. Jafar-Zanjani, and H. Mosallaei, "Rigorous space-time coupledwave analysis for patterned surfaces with temporal permittivity modulation," Opt. Mater. Express, vol. 9, no. 1, pp. 162-182, 2019.

[56] A. E. Cardin, S. R. Silva, S. R. Vardeny, W. J. Padilla, A. Saxena, A. J. Taylor, W. J. M. KortKamp, H.-T. Chen, D. A. R. Dalvit, and A. K. Azad, "Surface-wave-assisted nonreciprocity in spatio-temporally modulated metasurfaces," Nat Commun, vol. 11, no. 1, p. 1469, 2020.

[57] M. Albooyeh, D.-H. Kwon, F. Capolino, and S. A. Tretyakov, “Equivalent realizations of reciprocal metasurfaces: Role of tangential and normal polarization," Phys. Rev. B, vol. 95, no. 11, p. 115435, Mar. 2017.

[58] M. Selvanayagam and G. V. Eleftheriades, "Design And Measurement of Tensor Impedance Transmitarrays For Chiral Polarization Control," IEEE Trans Microw. Theory Techn, vol. 64, no. 2, pp. 414-428, Feb. 2016.

[59] B. P. Lathi and R. Green, "Linear Systems and Signals." New York, NY, USA: Oxford Univ. Press,, 2018, p. 170.

[60] A. V. Oppenheim, A. S. Willsky, and S. H. Nawab, Signals \& Systems, 2nd ed. Upper New Saddle River, NJ, USA: Prentice Hall, 1996.

[61] T. Claasen and W. Mecklenbrauker, "On stationary linear time-varying systems," IEEE Trans Circuits Syst, vol. 29, no. 3, pp. 169-184, 1982.

[62] L. E. Franks, "Signal Theory." Englewood Cliffs, NJ, USA: Prentice-Hall, 1969.

[63] L. A. Zadeh, “Frequency Analysis of Variable Networks," Proc IRE, vol. 38, no. 3, pp. 291-299, 1950 .

[64] F. Ding, A. Pors, and S. I. Bozhevolnyi, "Gradient metasurfaces: A review of fundamentals and applications," Rep Prog Phys, vol. 81, no. 2, p. 026401, 2017.

[65] C. Caloz, A. Alù, S. Tretyakov, D. Sounas, K. Achouri, and Z.-L. Deck-Léger, “Electromagnetic Nonreciprocity," Phys. Rev Appl, vol. 10, no. 4, p. 047001, 2018.

[66] S. Tretyakov, A. Sihvola, and B. Jancewicz, "Onsager-Casimir Principle and the Constitutive Relations of Bi-Anisotropic Media," J Electromagn Waves Appl, vol. 16, no. 4, pp. 573-587, 2002.

[67] A. Yaghjian, "An overview of near-field antenna measurements," IEEE Trans Antennas Propag, vol. 34, no. 1, pp. 30-45, Jan. 1986. 
[68] P. Goldsmith, “Quasi-optical techniques,” Proc IEEE, vol. 80, no. 11, pp. 1729-1747, Nov. 1992.

[69] N. Gagnon, J. Shaker, P. Berini, L. Roy, and A. Petosa, "Material characterization using a quasioptical measurement system," in Conf. Dig. Conf. Precis. Electromagn. Meas. IEEE, June 2002, pp. 104-105.

[70] J. P. S. Wong, M. Selvanayagam, and G. V. Eleftheriades, "Polarization Considerations for Scalar Huygens Metasurfaces and Characterization for 2-D Refraction," IEEE Trans Microw. Theory Techn, vol. 63, no. 3, pp. 913-924, Mar. 2015.

[71] I. Bruce, "ABCD transfer matrices and paraxial ray tracing for elliptic and hyperbolic lenses and mirrors," Eur. J. Phys., vol. 27, no. 2, pp. 393-406, Feb. 2006.

[72] B. E. A. Saleh and M. C. Teich, Fundamentals of Photonics, 3rd ed. Hoboken, USA: John Wiley \& Sons, Inc., 2019.

[73] V. Červený, "Expansion of a Plane Wave into Gaussian Beams," Studia Geophysica et Geodaetica, vol. 46, no. 1, pp. 43-54, Jan. 2002.

[74] K. Achouri and C. Caloz, “Design, concepts, and applications of electromagnetic metasurfaces," Nanophotonics, vol. 7, no. 6, pp. 1095-1116, 2018. 